\title{
Effective Action and Cluster Properties of the Abelian Higgs Model
}

\author{
Tadeusz Bałaban ${ }^{1, \star}$, John Z. Imbrie ${ }^{2, \star \star}$, and Arthur Jaffe ${ }^{2, \star \star \star}$ \\ ${ }^{1}$ Department of Mathematics, Northeastern University, Boston, MA 02115, USA \\ ${ }^{2}$ Harvard University, Cambridge, MA02138, USA
}

\begin{abstract}
We continue our program to establish the Higgs mechanism and mass gap for the abelian Higgs model in two and three dimensions. We develop a multiscale cluster expansion for the high frequency modes of the theory, within a framework of iterated renormalization group transformations. The expansions yield decoupling properties needed for a proof of exponential decay of correlations. The result of this analysis is a gauge invariant unit lattice theory with a deep Higgs potential of the shape required to exhibit the Higgs mechanism.
\end{abstract}

\section{Table of Contents}

1. Introduction . . . . . . . . . . . . . . . . . . . . . . . . . . . 258

2. Localized Kernels . . . . . . . . . . . . . . . . . . . . . . . . . . . . . 260

3. The First Renormalization Step . . . . . . . . . . . . . . . . . . . 265

4. The Inductive Hypothesis . . . . . . . . . . . . . . . . . . . . . . . . . . 273

5. Renormalization and Decoupling in the General Step . . . . . . . . . . . . . . 277

5.1. Renormalization Transformation . . . . . . . . . . . . . . . . . . . . 277

5.2. Restrictions on the Fields . . . . . . . . . . . . . . . . . . . . 278

5.3. First Gauge Field Translation . . . . . . . . . . . . . . . . . . . . . . 280

5.4. Gauge Transformation . . . . . . . . . . . . . . . . . . . . . . . . . 281

5.5. Second Gauge Field Translation . . . . . . . . . . . . . . . . . . . . . . . 283

5.6. Expansion with Respect to the Fluctuation Field . . . . . . . . . . . . . . 285

5.7. The Gaussian Normalization Factors . . . . . . . . . . . . . . . . . . 289

5.8. Scalar Field Translation. . . . . . . . . . . . . . . . . . . . . . . 295

5.9. Bounds on Fluctuation and Block Fields . . . . . . . . . . . . . . . . 296

5.10. The Interaction for the Fluctuation Fields . . . . . . . . . . . . . . . . . 298

5.11. Mayer Expansion I. . . . . . . . . . . . . . . . . . . . . . . . . . 299

5.12. Conditional Integration . . . . . . . . . . . . . . . . . . . . . . . 300

5.13. Decoupling of the Small Field Region. . . . . . . . . . . . . . . . . . . 303

5.14. Resummation and Extraction of the Perturbation Expansion . . . . . . . . . 307

5.15. Second Mayer Expansion and Scaling. . . . . . . . . . . . . . . . . . . 312

References . . . . . . . . . . . . . . . . . . . . . 314

* Research partially supported by the National Science Foundation under Grant DMS8602207 and by the Air Force Office of Scientific Research under Grant AFOSR-86-0229

$\star \star$ Alfred P. Sloan Research Fellow. Research partially supported by the National Science Foundation under Grants PHY-84-13285 and PHY-85-13554

$\star \star \star$ Research partially supported by the National Science Foundation under Grant PHY-8513554 


\section{Introduction}

We wish to establish the existence of a mass gap for the abelian Higgs model on the subspace of gauge invariant observables. Earlier work on this problem has led to a method to establish these results and to a partial solution $[1,2]$. Here we continue this study with the development of a multiscale expansion suitable for the problem. The basic formulation of the model is given in [2]. We consider an action function $S^{\varepsilon}$ which is defined for a gauge theory on a lattice with spacing $\varepsilon$. We use the Wilson form of lattice action, which is gauge invariant. Thus it is important to consider gauge invariant observables such as loop variables

$$
u(\gamma)=\exp \left[\sum_{b \in \gamma} i e \varepsilon A(b)\right]
$$

where $\gamma$ is a closed curve on the lattice, or string variables

$$
s(x, y, \Gamma)=\overline{\phi(x)} \exp \left(i e \varepsilon \sum_{b \in \Gamma} A(b)\right) \phi(y),
$$

where $\Gamma$ is a lattice curve from $x$ to $y$. These variables must be renormalized appropriately, by multiplying or subtracting $\varepsilon$-dependent terms. For gauge invariant operators (but not in general) we expect exponential clustering in the equilibrium state defined by $S^{\varepsilon}$. This state is given by the limit of normalized finite volume expectations

$$
\langle B\rangle=\frac{1}{Z} \int e^{-S^{\varepsilon}} B(u, \phi) \mathscr{D} u \mathscr{D} \phi .
$$

(We assume periodic boundary conditions, but this is not crucial since as a corollary we establish the existence of the infinite volume limit.) Thus for gauge invariant functions $B, C$ we expect

$$
|\langle B C\rangle-\langle B\rangle\langle C\rangle| \leqq O(1) \exp [-m \operatorname{dist}(B, C)],
$$

where $0<m$ and $\operatorname{dist}(B, C)$ denotes the distance between the supports of $B$ and $C$. For unit lattice models, (1.4) was established in [3] and here we investigate the corresponding estimates uniformly in the lattice spacing $\varepsilon$.

The exponential decay or mass gap is intimately connected with the Higgs mechanism. We see the Higgs mechanism at work through the evolution of the effective action as we proceed lower in momentum. The action on the $\varepsilon$-lattice appears almost massless, but as we approach the unit lattice, the Higgs potential exhibits a pronounced ring of minima at $|\phi|=\varrho_{0}$, which leads to a mass term for the gauge field. The apparently massless rotational degrees of freedom of $\phi$ can be gauged away.

To obtain decay, we need a convergent expansion with a small parameter. Thus, we restrict the coupling constants $(e, \lambda)$ to be sufficiently small in order to use cluster expansions. Such methods yield a nonperturbative analysis of the vacuum state, by explicitly displaying the exponential decay (1.4). Classically, the gauge field mass is of order $e / \lambda^{1 / 2}$, so we choose this ratio to be a fixed number of order unity.

The general ideas of these methods were described in our earlier papers $[1,2,4]$. Gauge invariance enters in a crucial way, both in the Higgs mechanism 
described above and in the control of ultraviolet divergences. By separating high and low momentum parts of the interaction in a gauge invariant way, we can choose convenient gauges to discuss renormalization of the high momentum part and to discuss the spectrum of the Hamiltonian in the low momentum region.

In the present paper, we consider clustering properties of the high frequency modes of the model. Our goal is an exact expression for the effective action on the unit lattice. The expression is complicated by the need to treat large field or large action regions differently from the perturbative, small field regions. The effective unit lattice theory is, however, similar in spirit to the one considered in [3]. A final cluster expansion will be performed on this theory in another paper, and the proof of clustering and of the existence of the mass gap will then be complete.

The dual requirements of clustering and of the renormalization group force us to develop a cluster expansion for each frequency mode separately. We formulate an inductive form of the model after $k$ renormalization group transformations, and then the bulk of this paper is devoted to clustering of the $(k+1)$-st mode in the next renormalization transformation.

The heart of our method is the way we accomplish changes of gauge without spoiling the exponential decoupling properties of the functional integral. We integrate out each frequency mode using a simple "tree" gauge on blocks (called axial gauge). After a number of modes have been integrated out, such gauges are not sufficiently regular to allow control of all the terms in the expansion. This is expected even in perturbation theory, where only gauges such as the Landau gauge are well behaved in the ultraviolet. Thus we must change the gauge in which those modes are expressed in the effective action. In keeping with the locality requirements of the cluster expansion, the change of gauge must be performed in patches, with slightly different changes on the overlaps. It turns out that the effects of the lack of alignment are small, and this way we avoid building up effects over long distances - something that tends to happen when changing gauges globally.

A similar problem occurs in our treatment of the effective unit lattice model. We have to change from the Landau gauge to the unitary gauge that is best suited for exhibiting the Higgs mechanism. Again, this must be accomplished without spoiling decoupling. Thus the method for changing gauges is the crucial aspect of our analysis, both for high momenta and for low momenta.

This paper is organized as follows. Having discussed Green's functions in [2] as global operators, we start by introducing localized forms of these operators which are better suited to the cluster expansion. We replace kernels $G(x, y)$ with kernels $G_{\text {loc }}(x, y)=G(x, y) \zeta(x, y)$, where $\zeta$ is smooth and supported in some neighborhood of $x=y$. This section also serves to review the roles of the various operators. We then briefly describe the cluster expansion in the first renormalization step. This leads to the formulation of the inductive hypothesis for the form of the model after $k$ renormalization steps. Finally, we describe the expansion in the general renormalization step. Usually we are able to prove the necessary convergence estimates as we describe each part of the expansion. This has the advantage of allowing us to consider each part in isolation, without worrying about the overall structure. Unfortunately this philosophy cannot be applied to the large field estimates. For these we find it necessary to postpone the estimates of convergence until integration over the final set of fields on the unit lattice. However, using the 
expected small factors arising from terms in the action with large fields, we show how convergence will eventually be obtained. We also assume estimates similar to those proven in [5] on the perturbation theory for this model.

\section{Localized Kernels}

In the previous paper [2], a number of operators arose from the application of renormalization transformations to the Gaussian approximation to the Higgs model. (Equation numbers from that paper will be prefixed here by $I$.) Exponential tails in the kernels of these approximately local operators are unavoidable. However, they are inconvenient for our analysis here, since they interfere with the decoupling of distant regions of space-time. Therefore, we introduce localized versions in which the tail has been cut off at a sufficiently large distance. In addition, for operators depending on an external gauge field, the dependence will be reduced to a bounded region. The use of the localized operators instead of the exact ones will introduce small error terms that are easily controlled.

Let us consider gauge field operators first. The minimizer $H_{k}$ maps unit lattice bond fields to $\eta$-lattice bond fields, $\eta=L^{-k}$. The configuration $A=H_{k} B$ minimizes the Landau gauge $\eta$-lattice gauge field action under the constraint $Q_{k} A=B$. We define $H_{k}$ starting with a large but fixed torus $T_{0, \varepsilon}$ of size $O\left(e^{-1}\right)$, say, in each lattice direction. [Recall our convention that subscripts on tori $T$ or $T_{0}$ indicate the lattice spacing (in this case $\varepsilon$ ); superscripts $(k)$ indicate the number of times the initial lattice has been decimated.] This avoids spurious dependence on the lattice $T_{\varepsilon}$ on which we put our model, and so simplifies the infinite volume limit. (Alternatively we could take the limit $T_{0, \varepsilon} \nearrow \in \mathbb{Z}^{d}$.) Construct a translation invariant localization function $\zeta_{k}$ such that

$$
\zeta_{k}\left(b, b^{\prime}\right)= \begin{cases}0, & \text { if } \quad \operatorname{dist}\left(b, b^{\prime}\right) \geqq \frac{1}{8} r\left(e_{k}\right), \\ 1, & \text { if } \quad \operatorname{dist}\left(b, b^{\prime}\right) \leqq \frac{1}{16} r\left(e_{k}\right),\end{cases}
$$

and such that $\zeta_{k}$ is a smooth function of $b$. Here $b \in T_{\eta}, b^{\prime} \in T_{1}^{(k)}$, and

$$
\begin{gathered}
e_{k}=\left(L^{k} \varepsilon\right)^{(4-d) / 2} e, \quad \lambda_{k}=\left(L^{k} \varepsilon\right)^{4-d} \lambda, \\
r\left(e_{k}\right)=\left|\log e_{k}^{-1}\right|^{r}, \quad r>1 .
\end{gathered}
$$

Then the localized version of $H_{k}$ has a kernel

$$
H_{k, \text { loc }}\left(b, b^{\prime}\right)=\zeta_{k}\left(b, b^{\prime}\right) H_{k}\left(b, b^{\prime}\right) .
$$

There is no ambiguity because $\zeta_{k}$ permits a sampling only of $b$ near $b^{\prime}$, relative to the size of $T_{0, \eta} ; H_{k}$ is also translation invariant. Since $\zeta_{k}$ is smooth, $H_{k, \text { loc }}$ inherits the regularity and decay properties of $H_{k}$, see (I.7.2.2). So we have

$$
\begin{gathered}
\left|H_{k, \text { loc }}\left(b, b^{\prime}\right)\right| \leqq c e^{-c \operatorname{dist}\left(b, b^{\prime}\right)}, \\
H_{k, \text { loc }}\left(b, b^{\prime}\right)=0 \text { for } \operatorname{dist}\left(b, b^{\prime}\right) \geqq \frac{1}{8} r\left(e_{k}\right), \\
\left|H_{k, \text { loc }}\left(b, b^{\prime}\right)-H_{k}\left(b, b^{\prime}\right)\right| \leqq e^{-c r\left(e_{k}\right)} e^{-c \operatorname{dist}\left(b, b^{\prime}\right)},
\end{gathered}
$$

and similarly for $\partial H_{k, \text { loc }}, \partial^{*} H_{k, \text { loc }}$, and for Hölder derivatives of $H_{k, \text { loc }}$ of order less than two. 
Next we consider $C^{(k)}$, the covariance of the $k$-th step gauge field. This is defined on the unit lattice $T_{0,1}^{(k)}$. First define

$$
\tilde{C}^{(k)}\left(b_{1}, b_{2}\right)= \begin{cases}C^{(k)}\left(b_{1}, b_{2}\right), & \text { if } \operatorname{dist}\left(b_{1}, b_{2}\right) \leqq \frac{1}{4} r\left(e_{k}\right), \\ 0, & \text { otherwise, }\end{cases}
$$

and extend by translation invariance to $T_{1}^{(k)}$. Then put

$$
C_{\mathrm{loc}}^{(k)}=\left(I-Q^{s *} Q\right) \widetilde{C}^{(k)}\left(I-Q^{*} Q^{s}\right) ;
$$

this insures that $C_{\text {loc }}^{(k)}$, like $C^{(k)}$, satisfies the constraints from the renormalization transformation and from the axial gauge conditions:

$$
\begin{aligned}
Q C_{\mathrm{loc}}^{(k)} & =C_{\mathrm{loc}}^{(k)} Q^{*}=0, \\
\sum_{b \in \Gamma_{y}, x} C_{\mathrm{loc}}^{(k)}\left(b, b_{0}\right) & =\sum_{b \in \Gamma_{y, x}} C_{\mathrm{loc}}^{(k)}\left(b_{0}, b\right)=0 .
\end{aligned}
$$

(See [2, Chap. 2] for definitions of the block averaging operators $Q, Q^{s}$, and $Q^{e}$.) We have estimates analogous to (2.5)-(2.7) for $C_{\text {loc }}^{(k)}$.

From $C_{\text {loc }}^{(k)}$ and $H_{k, \text { loc }}$ we construct a localized $\eta$-lattice gauge field propagator analogous to $\mathscr{D}_{k}$ :

$$
\mathscr{D}_{k, \mathrm{loc}}=\sum_{j=0}^{k-1} H_{j, \mathrm{loc}}^{L^{J} \eta} C_{\mathrm{loc}}^{(j), L^{\prime} \eta} H_{j, \mathrm{loc}}^{* L^{j}} \equiv \sum_{j=0}^{k-1} G_{\mathrm{loc}}^{(j), \eta} .
$$

Superscripts $L^{j} \eta, \eta$, etc. indicate the lattice spacing for operators rescaled to nonstandard lattices. This propagator derives its regularity and decay from that of $C_{\text {loc }}^{(k)}$ and $H_{k, \text { loc }}$. Thus

$$
\left|\left(\mathscr{D}_{k, \text { loc }} f\right)(b)\right| \leqq c e^{-c \operatorname{dist}(\operatorname{suppt} f, b)}\|f\|_{\infty},
$$

and similarly for derivatives of $\mathscr{D}_{k, \text { loc }}$ and Hölder derivatives of order less than 2. Furthermore,

$$
\mathscr{D}_{k, \text { loc }}\left(b_{1}, b_{2}\right)=0 \quad \text { for } \quad \operatorname{dist}\left(b_{1}, b_{2}\right) \geqq \frac{1}{2} r\left(e_{k}\right),
$$

and $\mathscr{D}_{k, \text { loc }}$ is close to $\mathscr{D}_{k}$, see (5.4.3) below.

The operator $\sigma_{k}$ gives the quadratic form for the $k$-th-step field strengths $f^{(k)}(p)=\left(i e_{h}\right)^{-1} \log u(p)$. As before we construct a localized operator on $T_{1}^{(k)}$ from $\sigma_{k}$ on $T_{0,1}^{(k)}$ :

$$
\sigma_{k, \text { loc }}\left(p_{1}, p_{2}\right)= \begin{cases}\sigma_{k}\left(p_{1}, p_{2}\right), & \text { if } \operatorname{dist}\left(p_{1}, p_{2}\right) \leqq \frac{1}{2 L} r\left(e_{k-1}\right), \\ 0, & \text { otherwise }\end{cases}
$$

where $p_{1}, p_{2}$ are plaquettes of $T_{1}^{(k)}$. Recall from [2] that

$$
\sigma_{k}=Q_{k}^{e}\left(I-\partial G_{k, A x} \partial^{*}\right) Q_{k}^{e *}=Q_{k}^{e}\left(I-\partial \mathscr{D}_{k} \partial^{*}\right) Q_{k}^{e *},
$$

the second equality following from the change of gauge, (I.5.2.6). Writing $\mathscr{D}_{k}$ in hierarchical form as in (2.12) and using the regularity of $H_{j}, 0 \leqq j<k$, we see that

$$
\left|\sigma_{k}\left(p_{1}, p_{2}\right)\right| \leqq c e^{-c \operatorname{dist}\left(p_{1}, p_{2}\right)} \text { for } \operatorname{dist}\left(p_{1}, p_{2}\right) \geqq c .
$$

[The rapid decay of the terms with small $j$ compensates for the scaling factors $\left(L^{j} \eta\right)^{-1}$.] For close $p_{1}, p_{2}$ the kernel of $\sigma_{k}$ can be large, of the order of $\eta^{-2}$. However, 
we shall only encounter situations where $f^{(k)}\left(p_{2}\right)=(\partial A)\left(p_{2}\right)$ for $p_{2}$ near $p_{1}$. Then we prove that

$$
\left(\sigma_{k} f^{(k)}\right)\left(p_{1}\right) \leqq\left\|f^{(k)}\right\|_{\infty}
$$

as follows. Write $f^{(k)}=\partial \square A+f^{\prime}$, where $\square$ is the characteristic function of a neighborhood of $p_{2}$. The distant part $\left(\sigma_{k} f^{\prime}\right)\left(p_{1}\right)$ is easily estimated by $\left\|f^{(k)}\right\|_{\infty}$ by (2.16). The near part is similarly bounded since $\sigma_{k}$ is a bounded operator on curls [2]. It was also shown in [2] that $\sigma_{k}$ is bounded from below. In view of (2.16) we have that

$$
\left|\sigma_{k, \operatorname{loc}}\left(p_{1}, p_{2}\right)-\sigma_{k}\left(p_{1}, p_{2}\right)\right| \leqq c e^{-c r\left(e_{k}\right)} e^{-c \operatorname{dist}\left(p_{1}, p_{2}\right)},
$$

so that $(2.16),(2.17)$ hold for $\sigma_{k, \text { loc }}$, and

as well.

$$
\sigma_{k, \text { loc }} \geqq c>0
$$

Another important kernel is the one generating the gauge transformation:

$$
\left(Q_{k}^{s *}-\mathscr{D}_{k} \partial^{*} Q_{k}^{e *} \partial\right) A=H_{k} A+\partial C_{k} A .
$$

The kernel $C_{k}$ is constructed from the basic gauge transformation $D_{k}$ which changes the minimizer from axial to Landau gauge (I.5.1.1):

$$
H_{k, \mathrm{Ax}} B=H_{k} B+\partial D_{k} B \text {. }
$$

By changing gauge in each term in the hierarchical sum defining $\mathscr{D}_{k}$ and applying (I.5.3.1), we obtain

$$
C_{k}=D_{k}+\sum_{j=0}^{k-1} D_{j}^{L^{\prime \eta}} C^{(j), L^{j} \eta} H_{j}^{* L^{\prime \prime} \eta} \partial^{*} Q_{k}^{e * \partial}
$$

The kernels of all these operators have an exponential decay on their respective length scales; for $D_{k}$ the required estimate is (I.7.2.4). The sum over $j$ is not well controlled for close points; this will not be important for us. For more distant points, however, the rapid decay of terms with small $j$ controls the scalings and the sum over $j$ to yield a uniform bound

$$
\left|C_{k}\left(x, b^{\prime}\right)\right| \leqq c e^{-c \operatorname{dist}\left(x, b^{\prime}\right)}, \quad \operatorname{dist}\left(x, b^{\prime}\right)>c .
$$

Here $x \in T_{0, \eta}, b^{\prime} \in T_{0,1}^{(k) *}$. Of course there is no uniform bound on $\partial C_{k}$. The localized version of $C_{k}$ is defined using another smooth cutoff:

$$
\zeta_{k}^{\prime}\left(x, b^{\prime}\right)=\left\{\begin{array}{lll}
1, & \text { for } & \operatorname{dist}\left(x, b^{\prime}\right) \leqq \frac{1}{4} r\left(e_{k}\right) \\
0, & \text { for } & \operatorname{dist}\left(x, b^{\prime}\right) \geqq \frac{1}{2} r\left(e_{k}\right)
\end{array} .\right.
$$

We then construct $C_{k, \text { loc }}$ on $T_{1}^{(k)}$ from $C_{k}$ on $T_{0,1}^{(k)}$ :

$$
C_{k, \text { loc }}\left(x, b^{\prime}\right)=\zeta_{k}^{\prime}\left(x, b^{\prime}\right) C_{k}\left(x, b^{\prime}\right) \text {. }
$$

Then $C_{k, \text { loc }}$ also satisfies (2.23) and

$$
\left|C_{k, \text { loc }}\left(x, b^{\prime}\right)-C_{k}\left(x, b^{\prime}\right)\right| \leqq e^{-c r\left(e_{k}\right)} e^{-c \operatorname{dist}\left(x, b^{\prime}\right)} .
$$

In the scalar field sector, we have the $\eta$-lattice propagators $G_{k}(\Omega, u)$ defined on subsets $\Omega \subset T_{\eta}$ with Neumann boundary conditions. To localize the dependence on 
$u$, we interpolate in a smooth fashion between operators with Neumann boundary conditions on small cubes. Let $\left\{\square_{\alpha}\right\}$ be the collection of $\frac{1}{2 \mathrm{~L}} r\left(e_{k-1}\right)$-cubes that can be built from cubes of size $M=O(1)$ as in [6]. Define

$$
\widetilde{G}_{k}\left(u ; x_{1}, x_{2}\right)=\sum_{\alpha} \lambda_{\alpha} G_{k}\left(\square_{\alpha}, u ; x_{1}, x_{2}\right)
$$

as a convex combination of Neumann propagators. The convex combination varies smoothly with $\left(x_{1}+x_{2}\right) / 2$; it involves at most $2^{d}$ terms and is concentrated on $\square_{\alpha}$ when $\left(x_{1}+x_{2}\right) / 2$ is near the center of $\square_{\alpha}$. We then put

$$
G_{k, \text { loc }}\left(u ; x_{1}, x_{2}\right)=\zeta_{k}^{\prime \prime}\left(x_{1}, x_{2}\right) \widetilde{G}_{k}\left(u ; x_{1}, x_{2}\right),
$$

where $\zeta_{k}^{\prime \prime}\left(x_{1}, x_{2}\right)$ is a smooth function of $x_{1}-x_{2}$,

$$
\zeta_{k}^{\prime \prime}\left(x_{1}, x_{2}\right)=\left\{\begin{array}{lll}
0, & \text { if } & \left|x_{1}-x_{2}\right| \geqq \frac{1}{4 L} r\left(e_{k-1}\right) \\
1, & \text { if } & \left|x_{1}-x_{2}\right| \leqq \frac{1}{8 L} r\left(e_{k-1}\right)
\end{array} .\right.
$$

The boundary conditions are always at a distance $O\left(r\left(e_{k}\right)\right)$ from $x_{1}, x_{2}$, so a straightforward application of the random walk expansion of [6] shows that

$$
\begin{gathered}
\left|\left(G_{k, \text { loc }}(u) f\right)(x)\right| \leqq c e^{-c \operatorname{dist}(\operatorname{suppt} f, x)}\|f\|_{\infty}, \\
\left|\left(G_{k, \text { loc }}(u) f-G_{k}(\Omega, u) f\right)(x)\right| \leqq e^{-c r\left(e_{k}\right)} e^{-c \operatorname{dist}(\operatorname{suppt} f, x)}\|f\|_{\infty},
\end{gathered}
$$

for $\operatorname{dist}\left(x, \Omega^{c}\right) \geqq O\left(r\left(e_{k}\right)\right)$. [Each $G_{k}\left(\square_{x}, u\right)$ is close to $G_{k}(\Omega, u)$ for the relevant $x_{1}, x_{2}$, therefore the convex combination and $G_{k, \text { loc }}$ are close also.] We assume that $u$ is smooth in the $\square_{\alpha}$ 's entering the sum in (2.27); for (2.31) we assume smoothness throughout the subset $\Omega \subset T_{\eta}$. This means that in a neighborhood of each $\square_{\alpha}$ there exists an $A, \lambda$ such that

$$
u=\exp \left[i e_{k} \eta(A+\partial \lambda)\right] \text { with }|\partial A|,\left|\partial^{*} A\right| \leqq O\left(p\left(e_{k}\right)\right) .
$$

Here

$$
p\left(e_{k}\right)=\left|\log e_{k}^{-1}\right|^{p}, \quad p=O(1)
$$

is our logarithmic scale for small fields. Bounds analogous to (2.30), (2.31) hold for covariant derivatives and Hölder derivatives of $G_{k \text { loc }}(u)$ of order less than two.

We use $G_{k, \text { loc }}$ to define a localized quadratic form for scalar fields,

$$
\Lambda_{k, \text { loc }}(u)=a_{k} I-a_{k}^{2} Q_{k}(u) G_{k, \text { loc }}(u) Q_{k}^{*}(u) .
$$

Here we have simply replaced $G_{k}(\Omega, u)$ with $G_{k, \text { loc }}$ in the definition of $\Delta_{k}(\Omega, u)$; see (I.4.6.4). Hence

$$
\begin{aligned}
& \left|\Delta_{k, \text { loc }}\left(u ; x_{1}, x_{2}\right)-\Delta_{k}\left(\Omega, u ; x_{1}, x_{2}\right)\right| \\
& \leqq e^{-c r\left(e_{k}\right)} e^{-c\left|x_{1}-x_{2}\right|} \text { for } \operatorname{dist}\left(\left\{x_{1}, x_{2}\right\}, \Omega^{c}\right)>O\left(r\left(e_{k}\right)\right), \\
& \left|\Delta_{k, \text { loc }}\left(u ; x_{1}, x_{2}\right)\right| \leqq c e^{-c\left|x_{1}-x_{2}\right|}, \\
& \Delta_{k, \text { loc }}\left(u ; x_{1}, x_{2}\right)=0 \text { for }\left|x_{1}-x_{2}\right| \geqq \frac{1}{2 L} r\left(e_{k-1}\right) .
\end{aligned}
$$


Again we assume $u$ is smooth in the relevant regions; $\Delta_{k \text {, loc }}\left(u ; x_{1}, x_{2}\right)$ depends on $u$ only in an $O\left(r\left(e_{k}\right)\right)$-neighborhood of $x_{1}, x_{2}$. Finally, in view of (2.35), the lower bound (I.7.3.2) applies to $\Delta_{k, \text { loc }}(u)$ as well. Let $\phi$ be supported in a region having an $r\left(e_{k}\right)$ neighborhood where $u$ is smooth. Then

$$
\left\langle\phi, \Delta_{k, \operatorname{loc}}(u) \phi\right\rangle \geqq c \sum_{b \in T_{1}^{(k) *}}\left|u\left(\left\langle b_{-}, b_{+}\right\rangle\right) \phi\left(b_{+}\right)-\phi\left(b_{-}\right)\right|^{2}-c e_{k}^{2} p\left(e_{k}\right)^{2} \sum_{x \in T_{1}^{(k)}}|\phi(x)|^{2} .
$$

Finally, we need to construct a localized version of

$$
C_{\Lambda}^{(k)}(\Omega, u)=\left[\left.\left(\Delta_{k}(\Omega, u)+a L^{-2} Q(u)^{*} Q(u)\right)\right|_{\Lambda}\right]^{-1},
$$

the single-scale propagator for the scalar field in the $k$-th step. We have already replaced $\Delta_{k}(\Omega, u)$ with $\Delta_{k, \text { loc }}(u)$. Let us assume $u$ is smooth in a neighborhood of $\Lambda$, the region for the Dirichlet boundary conditions in (2.39). We define

$$
C_{\Lambda}^{(k)}(u)=\left[\left(\Delta_{k, \text { loc }}(u)+\left.a L^{-2} Q(u)^{*} Q(u)\right|_{\Lambda}\right]^{-1} .\right.
$$

This is of course a nonlocal operator, but by $(2.38), C_{A}^{(k)}(u)^{-1}$ is bounded below and a random walk expansion as in [6] can be used to prove that

$$
\left|C_{A}^{(k)}\left(u ; x_{1}, x_{2}\right)\right| \leqq c e^{-c\left|x_{1}-x_{2}\right|}
$$

We shall actually use a convenient resummation of this expansion. The basic expansion has the form

$$
C_{\Lambda}^{(k)}(u)=\sum_{\omega} C_{\omega}
$$

where $\omega$ is a walk on a lattice of spacing $M=O(1)$. We define the localized form of $C_{\Lambda}^{(k)}(u)$ to be

$$
C_{\Lambda, \text { loc }}^{(k)}\left(u ; x_{1}, x_{2}\right)=\sum_{\omega}^{\prime} C_{\omega}\left(x_{1}, x_{2}\right)
$$

where the prime indicates that only $\omega$ remaining within $\frac{1}{4} r\left(e_{k}\right)$ of $x_{1}, x_{2}$ are included. Let $X$ be a connected union of $r\left(e_{k}\right)$-cubes, and let $X^{0}$ be the cubes of $X$ not at the boundary of $X$. We define

$$
C_{\Lambda, X}^{(k)}\left(u ; x_{1}, x_{2}\right)=\sum_{\omega}^{X} C_{\omega}\left(x_{1}, x_{2}\right)
$$

where the sums runs over walks not included in $\Sigma^{\prime}$, which remain within $X^{0}$ and which intersect each cube of $X^{0}$. Then we define

$$
C_{\Lambda}^{(k)}(u)=C_{\Lambda, \operatorname{loc}}^{(k)}(u)+\sum_{X} C_{\Lambda, X}^{(k)}(u)
$$

and the convergence and locality properties of the random walk expansion imply the following facts about these operators. The local part $C_{\Lambda, \operatorname{loc}}^{(k)}\left(u ; x_{1}, x_{2}\right)$ depends only on $u$ in an $O\left(r\left(e_{k}\right)\right)$ neighborhood of $x_{1}, x_{2}$; it vanishes for $\left|x_{1}-x_{2}\right|>\frac{1}{2} r\left(e_{k}\right)$ and is bounded as in (2.41). The operator $C_{\Lambda, X}^{(k)}(u)$ depends only on $u$ in $X$. It vanishes unless both arguments are in $X$, and is estimated as follows:

$$
\left|C_{\Lambda, X}^{(k)}\left(u ; x_{1}, x_{2}\right)\right| \leqq e^{-c r\left(e_{k}\right)|X|}
$$


Here and elsewhere, $|X|$ refers to the number of $r\left(e_{k}\right)$-cubes in $X$, not the volume of $X$. This estimate can be summed over all connected sets $X$ to show that

$$
\left|C_{\Lambda, \text { loc }}^{(k)}\left(u ; x_{1}, x_{2}\right)-C_{\Lambda}^{(k)}\left(u ; x_{1}, x_{2}\right)\right| \leqq e^{-c r\left(e_{k}\right)} e^{-c\left|x_{1}-x_{2}\right|} .
$$

If $X$ does not intersect $\Lambda^{c}$, then $C_{\Lambda, X}^{(k)}(u)$ does not depend on $\Lambda$; neither does $C_{\Lambda, \text { loc }}^{(k)}\left(u ; x_{1}, x_{2}\right)$ depend on $\Lambda$ if $\operatorname{dist}\left(\left\{x_{1}, x_{2}\right\}, \Lambda^{c}\right)>\frac{1}{2} r\left(e_{k}\right)$. In this case we write it as

$$
C_{\text {loc }}^{(k)}\left(u ; x_{1}, x_{2}\right)=C_{\Lambda, \text { loc }}^{(k)}\left(u ; x_{1}, x_{2}\right), \quad \Lambda \text { large enough . }
$$

Note that all operators introduced through random walk expansions of $C_{A}^{(k)}(u)$ or $G_{k}(\Omega, u)$ transform properly under gauge transformations, that is, by the difference of the gauge transformation between the points of evaluation of the kernel.

Lastly we note that the single-step covariance for the gauge field can be given a random walk expansion analogous to (2.45), with similar estimates:

$$
C_{\Lambda}^{(k)}=C_{\Lambda, \text { loc }}^{(k)}+\sum_{X} C_{\Lambda, X}^{(k)}
$$

\section{The First Renormalization Step}

In this section we briefly and informally describe the sequence of operators performed in the first renormalization step. This will serve to orient the reader in the more detailed descriptions for the general step, and it will motivate the inductive hypothesis for the general step. Most estimates will not be discussed here, since they are special cases of those proven for the general step. We avoid formulae in favor of verbal descriptions, except for the first few operations, which are special to the first step.

We wish to give an expansion for the partition function, or for an unnormalized expectation of an observable $F$. Thus we consider

$$
[F]=\int \mathscr{D} u \mathscr{D} \phi e^{-S^{\varepsilon}(u, \phi)} F,
$$

where $F$ is a gauge-invariant function, a product of terms like $|\phi(x)|^{2}$, $\bar{\phi}\left(b_{-}\right) u(b) \phi\left(b_{+}\right), \operatorname{Re}\left(i e \varepsilon^{2}\right)^{-1}(u(p)-1)$. Each such term may need to have an appropriate constant subtracted in order to obtain $\varepsilon$-independent bounds on the full expectation

$$
\langle F\rangle=[F] /[1] .
$$

These "Wick ordering" constants are given by perturbation theory to a low order, and will be discussed carefully in a subsequent paper on the perturbation expansions.

The action on $T_{\varepsilon}$, the $\varepsilon$-lattice, is

$$
S^{\varepsilon}(u, \phi)=\sum_{p \in T_{\varepsilon}^{* *}} \varepsilon^{d} \frac{1}{e^{2} \varepsilon^{4}}[1-\operatorname{Re} u(p)]+\frac{1}{2}\left\langle\phi,-\Delta_{u}^{\varepsilon} \phi\right\rangle+\sum_{x \in T_{\varepsilon}} \varepsilon^{d} P(\phi(x))+E_{0}+E_{1} .
$$

Here $-\Delta_{u}^{\varepsilon}=D_{u}^{\varepsilon *} D_{u}^{\varepsilon}$, and

$$
P(\phi)=\lambda|\phi|^{4}-\frac{1}{4}|\phi|^{2}+\frac{1}{64 \lambda}-\frac{1}{2} \delta m^{2}|\phi|^{2} .
$$


We have taken the bare scalar field mass [coming from the radial curvature of $P(\phi)$ ] equal to 1 ; other values can be achieved by scalings. We have included a mass renormalization $\delta m^{2}=\delta m^{2}(e, \lambda, \varepsilon)$ and a vacuum energy renormalization $E_{1}$ $=E_{1}\left(e, \lambda, \varepsilon,\left|T_{\varepsilon}\right|\right)$. The constant $E_{0}=E_{0}\left(\varepsilon, T_{\varepsilon}\right)$ normalizes the integral (3.1) so that

$$
\lim _{\lambda, e \rightarrow 0}[1]=1 \text {. }
$$

We will be considering subsets $X$ of the lattice $T_{a}^{(k)}$ obtained by decimating $T_{\varepsilon}$ $k$-times and scaling the resulting lattice spacing to $a$. We denote by $X^{*}$ the set of bonds with both endpoints in $X$; then $X^{* *}$ denotes the set of plaquettes with all four corners in $X$. A superscript $c$ denotes complement, so that $X^{c}=T_{a}^{(k)} \backslash X, X^{* c}$ $=T_{a}^{(k) *} \backslash X^{*}$, etc. Thus $X^{c * c}$ includes bonds with one or both endpoints in $X$.

We rescale our expressions from $T_{\varepsilon}$ to the unit lattice $T_{1}$. The scalar field is multiplied by $\varepsilon^{-(d-2) / 2}$, and we have

$$
\begin{gathered}
{[F]=\int \mathscr{D} u \mathscr{D} \phi \varrho_{0}(u, \phi),} \\
\varrho_{0}(u, \phi)=F \exp \left[-\sum_{p \in T_{1}^{* *}} e_{0}^{-2}(1-\operatorname{Re} u(p))-\frac{1}{2}\left\langle\phi,-\Delta_{u} \phi\right\rangle\right. \\
\left.-\sum_{x \in T_{1}} P_{0}(\phi(x))-\sum_{x \in T_{1}} \frac{1}{2} \delta m^{2} \varepsilon^{2}|\phi(x)|^{2}-\mathscr{E}_{0}-E_{1}\right] .
\end{gathered}
$$

Here $P_{0}$ is the first in a sequence of scalar potentials forming the dominant term after $k$ steps:

$$
P_{k}(\phi)=\lambda_{k}|\phi|^{4}-\frac{1}{4}\left(L^{k} \varepsilon\right)^{2}|\phi|^{2}+\frac{1}{64 \lambda}\left(L^{k} \varepsilon\right)^{d}
$$

We use a rescaled coupling constant

$$
\lambda_{k}=\left(L^{k} \varepsilon\right)^{4-d} \lambda
$$

and since $e^{2} / \lambda=O(1)$ we have also $e_{k}^{2} / \lambda_{k}=O(1)$, by (2.2). The constant $\mathscr{E}_{0}$ includes the scaling factors,

$$
\mathscr{E}_{0}=E_{0}-(d-2)\left|T_{1}\right| \log \varepsilon^{-1}
$$

Each factor $\phi$ in $F$ acquires a factor $\varepsilon^{-(d-2) / 2}$, but we use the same notation. Ultimately these scaling factors will be cancelled by successive rescalings back to the original scale.

We begin to compute $[F]$ by integrating over $u, \phi$ under constraints given by the block fields $v, \psi$ on the $L$-lattice. This is the renormalization transformation, described in the previous paper. With the gauge fix $\delta_{\mathrm{Ax}}(u)$, it takes the density $\varrho_{0}(u, \phi)$ to

$$
\begin{aligned}
\varrho_{1}^{L}(v, \psi)= & \int \mathscr{D} u \mathscr{D} \phi \delta(v / Q u) \delta_{\mathrm{Ax}}(u) F \exp \left[-\sum_{p} e_{0}^{-2}(1-\operatorname{Re} u(p))\right. \\
& -\frac{1}{2} a L^{-2}\langle\psi-Q(u) \phi, \psi-Q(u) \phi\rangle-\frac{1}{2}\left\langle\phi,-\Delta_{u} \phi\right\rangle \\
& \left.-\sum_{x} P_{0}(\phi(x))-\sum_{x} \frac{1}{2} \delta m^{2} \varepsilon^{2}|\phi(x)|^{2}-\mathscr{E}_{0}-E^{(0)}-E_{1}\right] .
\end{aligned}
$$


Here we define

$$
E^{(0)}=-\left|T_{1}^{(1)}\right| \log \left(a L^{d-2} / 2 \pi\right),
$$

which normalizes the transformation so that

$$
[F]=\int d v d \psi \varrho_{1}^{L}(v, \psi) .
$$

The first operation is a decomposition of the lattice into large and small field regions. This is accomplished by means of a partition of unity,

$$
1=\sum_{\Lambda_{0}^{(0)}} \zeta_{\Lambda_{0}^{(0) c}} \chi_{\Lambda_{0}^{(0)}}
$$

Here $\Lambda_{0}^{(0)} C T_{1}$ is the small field region. It is composed of $r\left(e_{0}\right)$-cubes, in each of which the factor $\chi_{\Lambda_{0}^{(0)}}$ enforces the following conditions:

$$
\begin{gathered}
\left|D_{u} \phi\right| \leqq p\left(e_{0}\right), \quad|\psi-Q(u) \phi| \leqq p\left(e_{0}\right), \quad|\phi| \leqq \lambda_{0}^{-1 / 4} p\left(e_{0}\right) \\
\left|f^{(0)}(p)\right| \leqq p\left(e_{0}\right), \quad \text { where } \quad f^{(0)}(p)=\left(i e_{0}\right)^{-1} \log u(p) .
\end{gathered}
$$

The factor $\zeta_{A_{0}^{(0) c}}$ forces at least one of these conditions to be violated somewhere in each $r\left(e_{0}\right)$-cube of $\Lambda_{0}^{(0) c}$.

Later in this step we will introduce sets $\Lambda_{1}^{(0)}, \Lambda_{2}^{(0)}$, etc., which are obtained from $\Lambda_{0}^{(0)}$ either by deleting $r\left(e_{0}\right)$-cubes at the boundary of $\Lambda_{0}^{(0)}$, or by deleting $r\left(e_{0}\right)$-cubes covering regions with "irrelevant" terms from the expansions. (These are terms bounded by a high power of rescaled coupling constants.) In the $k$-th step we will introduce analogous small field sets $\Lambda_{0}^{(k)}, \Lambda_{1}^{(k)}$, etc.

In the previous paper, we worked with the basic quadratic form $\langle\partial A, \partial A\rangle$. This is obtained now by expanding the Wilson action in powers of $e_{0}$. In $\Lambda_{0}^{(0) * *}$ we have small $f^{(0)}$, so we write

$$
\begin{aligned}
e_{0}^{-2}[1-\operatorname{Re} u(p)] & =\frac{1}{2} f^{(0)}(p)^{2}+\sum_{n=2}^{\bar{n} / 2}(-1)^{n} \frac{e_{0}^{2 n-2}\left(f^{(0)}(p)\right)^{2 n}}{(2 n) !}+W_{0}(p) \\
& =\frac{1}{2} f^{(0)}(p)^{2}+V_{0}(p)+W_{0}(p)
\end{aligned}
$$

We consider the expansion up to order $\bar{n}$ in $e_{0}$ explicitly, the remainder is called "irrelevant" because it is bounded by $c e_{0}^{\bar{n}} p\left(e_{0}\right)^{\bar{n}+2} \leqq e \varepsilon^{d+1}$ for $\bar{n}$ large enough. The first term, summed over $\Lambda_{0}^{(0) * *}$, gives rise to the quadratic form $\frac{1}{2}\left\langle\Lambda_{0}^{(0) * *} f^{(0)}\right.$, $\left.\Lambda_{0}^{(0) * *} f^{(0)}\right\rangle$. (We use $\Lambda^{* *}$ to denote the set of plaquettes with all four corners in $\Lambda$; $\Lambda^{*}$ denotes the bonds with both endpoints in $\Lambda$. The same symbols are used for the corresponding characteristic functions.) The low order terms in $e_{0}$ are new interaction vertices.

For factors $\left(i e \varepsilon^{2}\right)^{-1}(u(p)-1)=\left(i e_{0}\right)^{-1} \varepsilon^{-d / 2}(u(p)-1)$ in the observable $F$, $p \in \Lambda_{0}^{(0) * *}$, we expand:

$$
\begin{aligned}
(i e(\varepsilon))^{-1} \varepsilon^{-d / 2}(u(p)-1) & =\sum_{n=1}^{\infty} \varepsilon^{-d / 2} \frac{\left(i e_{0}\right)^{n-1}}{n !}\left(f_{0}(p)\right)^{n} \\
& =F_{\mathrm{rel}}(p)+F_{\mathrm{irr}}(p) .
\end{aligned}
$$

The first three terms are relevant (for observables this means they do not go to zero with $\varepsilon$.) The others are included in $F_{\text {irr }}(p)$. (Our use of the terms "relevant" and 
"irrelevant" is different from standard renormalization group language.) We sum over these two terms for $p \in \pi(F) \cap \Lambda_{0}^{(0) * *}$, where $\pi(F)$ is the set of plaquettes having factors $\operatorname{Re}\left(i e \varepsilon^{2}\right)^{-1}(u(p)-1)$ in $F$ (with multiplicity). Denote by $\beta(F), \xi(F)$ the bond, sites having factors : $\bar{\phi}\left(b_{-}\right) u(b) \phi\left(b_{+}\right):$, or $:|\phi(x)|^{2}:$ in $F$. The result is the following expansion for $F$ :

$$
\begin{aligned}
F= & \sum_{S_{\pi} \subset \pi(F) \cap \Lambda_{0}^{(0) * * *}} \prod_{p \in S_{\pi}} F_{\mathrm{irr}}(p) \prod_{p \in S_{\pi}^{c}} F_{\mathrm{rel}}(p) \prod_{p \in \pi(F) \backslash \Lambda_{0}^{(0) * *}} \\
& \times \operatorname{Re}\left(i e \varepsilon^{2}\right)^{-1}(u(p)-1) \prod_{b \in \beta(F)}: \bar{\phi}\left(b_{-}\right) u(b) \phi\left(b_{+}\right): \prod_{x \in \xi(F)}:|\phi(x)|^{2}: .
\end{aligned}
$$

The irrelevant part of the gauge field action is Mayer-expanded:

$$
\exp \left[-\sum_{p \in \Lambda_{0}^{(0) * *}} W_{0}(p)\right]=\sum_{S_{p} \subset A_{0}^{(0) * * *}} \prod_{p \in S_{p}}\left(e^{-W_{0}(p)}-1\right) .
$$

We group together large-field regions and regions with irrelevant terms. Anticipating the structure of the induction, we define $A_{13}^{(-1) c}$ as the union of $r\left(e_{0}\right)$-cubes covering $\Lambda_{0}^{(0) c}$ and all plaquettes in $S_{p}$ or $S_{\pi}$. We divide $\Lambda_{13}^{(-1) c}$ into connected components $\left\{X_{\omega}\right\}$, and define

$$
\begin{aligned}
& g_{0}\left(X_{\omega}\right)=\sum_{S_{\pi} \subset \pi(F) \cap \Lambda_{0}^{(0) * *} \cap X_{\omega}^{* *}} \sum_{S_{p} \subset A_{0}^{(0) * *} \cap X_{\omega}^{* *}} \prod_{p \in S_{\pi}} \\
& \times F_{\text {irr }}(p) \prod_{p \in S_{-\tau}^{c} \cap X_{\omega}^{* *}} F_{\text {rel }}(p) \prod_{p \in\left(\pi(F) \cap X_{\sigma}^{*}\right) \backslash \Lambda_{0}^{(0) * * *}} \\
& \times \operatorname{Re}\left(i e \varepsilon^{2}\right)^{-1}(u(p)-1) \prod_{b \in \beta(F) \cap X_{t}^{*}}: \bar{\phi}\left(b_{-}\right) u(b) \phi\left(b_{+}\right): \\
& \times \prod_{X \in \xi(F) \cap X_{\omega}}:|\phi(x)|^{2}: \prod_{p \in S_{p}}\left(e^{-W_{0}(p)}-1\right)
\end{aligned}
$$

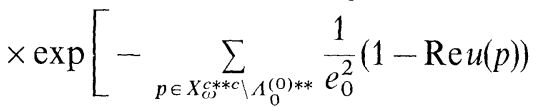

$$
\begin{aligned}
& \text { - } \left.\sum_{x \in X_{(\supset) \Lambda \Lambda_{0}^{(0)}}}\left(P_{0}(\phi(x))+\frac{1}{2} \delta m^{2} \varepsilon^{2}|\phi(x)|^{2}+E_{1}(x)\right)\right] \text {. }
\end{aligned}
$$

We have written $E_{1}=\sum_{x \in T_{1}} E_{1}(x), E_{1}(x)$ defined by fixing one vertex at $x$ for each diagram defining $E_{1}$.

The remaining $r\left(e_{0}\right)$-cubes covering the support of $F$ are divided into connected components $\left\{X_{0}\right\}$, and we put

$$
\begin{aligned}
F_{0, \text { loc }}\left(X_{\sigma}\right)= & \prod_{p \in X_{\sigma *}^{*} \cap \pi(F)} F_{\text {rel }}(p) \prod_{b \in X_{\sigma}^{*} \cap \beta(F)}: \phi\left(b_{-}\right) u(b) \phi\left(b_{+}\right): \\
& \times \prod_{x \in X_{\sigma} \cap \xi(F)}:|\phi(x)|^{2}: .
\end{aligned}
$$

Our density now takes the following form:

$$
\begin{aligned}
\varrho_{1}^{L}(v, \psi)= & \sum_{\Lambda_{0}^{(0)}} \sum_{\left\{X_{\omega}\right\}} \int \mathscr{D} u \mathscr{D} \phi \delta(v / Q u) \delta_{\mathrm{Ax}}(u) \zeta_{\Lambda_{0}^{(0)}} \chi_{\Lambda_{0}^{(0)}} \\
& \times \prod_{(=)} g_{0}\left(X_{\omega}\right) \prod_{\sigma} F_{0, \operatorname{loc}}\left(X_{\sigma}\right) \exp \left[-\frac{1}{2}\left\langle\Lambda_{0}^{(0) * *} f^{(0)}, \Lambda_{0}^{(0) * *} f^{(0)}\right\rangle\right. \\
& -\frac{1}{2} a L^{-2}\langle\psi-Q(u) \phi, \psi-Q(u) \phi\rangle-\frac{1}{2}\left\langle\phi,\left(-\Delta_{u}\right) \phi\right\rangle \\
& \left.-\mathscr{P}_{0, \operatorname{loc}}\left(\Lambda_{0}^{(0)}\right)-\mathscr{E}_{0}-E^{(0)}\right],
\end{aligned}
$$


where the basic interaction terms have been included in

$$
\mathscr{P}_{0, \text { loc }}\left(\Lambda_{0}^{(0)}\right)=\sum_{x \in \Lambda_{0}^{(0)}}\left(P_{0}(\phi(x))+\frac{1}{2} \delta m^{2} \varepsilon^{2}|\phi(x)|^{2}+E_{1}(x)\right)+\sum_{p \in \Lambda_{0}^{(0) * *}} V_{0}(p) .
$$

These expressions have a form similar to that of the inductive hypothesis for the general renormalization step, introduced in Sect. 4.1. Nevertheless, we continue in an informal fashion with the first step, in order to outline the conceptual ideas whose details are treated in the general case of the next chapter.

We begin with a translation of the gauge field which takes the block field $v$ out of the $\delta$-functions of the renormalization transformation. Thus we put

$$
\begin{aligned}
u_{b} & = \begin{cases}u_{b}^{\prime} u_{b^{\prime}}, & \text { if } b \in B^{s}\left(b^{\prime}\right) \cap \Lambda_{1}^{(0) *}, \\
u_{b}^{\prime}, & \text { otherwise. }\end{cases} \\
& \equiv u_{b}^{\prime}\left(\Lambda_{1}^{(0) *} Q^{s *} v\right)_{b},
\end{aligned}
$$

where the prefactor $\Lambda_{1}^{(0) *}$ indicates that what follows is present only for $b \in \Lambda_{1}^{(0) *}$. From the restrictions on the fields, we have that $u_{b}^{\prime}=e^{i e_{0} A_{b}^{\prime}}$, with $\left|A_{b}^{\prime}\right| \leqq c p\left(e_{0}\right)$ in $\Lambda_{1}^{(0) *}$. The axial gauge $\delta$-functions are invariant under this translation. In $\Lambda_{1}^{(0)}$, $\delta(v / Q u)$ becomes proportional to $\delta\left(Q A^{\prime}\right)$.

In the general step, a gauge transformation is needed at this point. However, it is unnecessary here.

The quadratic form for the gauge field in $\Lambda_{1}^{(0) * *}$ is

$$
\begin{aligned}
& \left\langle\Lambda_{1}^{(0) * *} f^{(0)}, \Lambda_{1}^{(0) * *} f^{(0)}\right\rangle \\
& \quad=\left\langle\Lambda_{1}^{(0) * *}\left(\partial A^{\prime}+L^{-2} Q^{e * f}\right), \Lambda_{1}^{(0) * *}\left(\partial A^{\prime}+L^{-2} Q^{e * f}\right)\right\rangle,
\end{aligned}
$$

where

$$
f(p)=\left(i e_{0}\right)^{-1} \log v(p) .
$$

A second translation is needed to remove the term linear in $A^{\prime}$. We put

$$
A^{\prime}=A^{(0)}-\Lambda_{4}^{(0) *} L^{-2} C_{\mathrm{loc}}^{(0)} \partial^{*} Q^{e * f},
$$

which does not precisely eliminate the linear term. However, it is local, and away from $\partial \Lambda_{4}^{(0)}$ the linear term is extremely small. If we neglect terms at $\partial \Lambda_{4}^{(0)}$ and localized terms of the order of $e^{-c r\left(e_{0}\right)}$, we obtain the main quadratic forms for block and fluctuation fields:

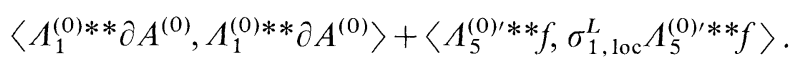

The prime denotes decimation (taking the corners of blocks only); the superscript $L$ indicates the block lattice spacing.

Let us write the background gauge field in $\Lambda_{3}^{(0) *}$ in terms of $A^{(0)}$. It is

$$
u=\left(Q^{s *} v\right) \exp \left[i e_{0}\left(A^{(0)}-\Lambda_{4}^{(0) *} L^{-2} C_{\mathrm{loc}}^{(0)} \partial^{*} Q^{e * f}\right)\right] .
$$

We wish to expand in $A^{(0)}$ in the scalar field quadratic forms, and in the observables $F_{0, \text { loc }}$, where this gauge field appears. This will give us scalar field forms that depend only on the block gauge field. Let $\theta_{0}$ be the characteristic function of $\Lambda_{6}^{(0) *}$. We expand in $\theta_{0} A^{(0)}$. For terms of zeroth order in $\theta_{0} A^{(0)}$ we have 
a background gauge field

$$
u_{1}=\left(\Lambda_{1}^{(0) *} Q^{s *} v\right)\left(\Lambda_{6}^{(0) * c} u^{(0)}\right) \exp \left(i e_{0} \Lambda_{4}^{(0) *} L^{-2} C_{\mathrm{loc}}^{(0)} \partial^{*} Q^{e * f}\right) .
$$

Here $u^{(0)}=\exp \left(i e_{0} A^{(0)}\right)$. Terms of first or higher order in $\theta_{0} A^{(0)}$ will be treated as interactions.

The expansion yields for the scalar field forms

$$
\begin{aligned}
& \frac{1}{2} a L^{-2}\langle\psi-Q(u) \phi, \psi-Q(u) \phi\rangle+\frac{1}{2}\left\langle\phi,-\Delta_{u} \phi\right\rangle=\frac{1}{2} a L^{-2}\left\langle\psi-Q\left(u_{1}\right) \phi, \psi-Q\left(u_{1}\right) \phi\right\rangle \\
& \quad+\frac{1}{2}\left\langle\phi,-\Delta_{u_{1}} \phi\right\rangle+R^{(0)}\left(u_{1}, \theta_{0} A^{(0)}\right)+\sum_{\square} W_{1}^{(0)}(\square),
\end{aligned}
$$

where $R^{(0)}$ contains the first $\bar{n}$ orders in $A^{(0)}$ (or in $e_{0}$ ) and the higher order, irrelevant, local terms are incorporated in $W_{1}^{(0)}(\square)$. (These terms can always be localized to some $r\left(e_{0}\right)$-cube $\square$.) Similarly each factor $F_{0, \text { loc }}\left(X_{\sigma}\right)$ is written as a sum of two terms: the first $\bar{m}$ orders in $e_{0}$, and the remainder which is bounded uniformly in $\varepsilon$ for an appropriate choice of $\bar{m}$.

The next step is a scalar field translation to remove the term linear in $\phi$ in (3.29). Again we make a local translation,

$$
\phi=\phi^{(0)}+a L^{-2} \Lambda_{7}^{(0)} C_{\mathrm{loc}}^{(0)}\left(u_{1}\right) Q^{*}\left(u_{1}\right) \psi .
$$

Neglecting terms at $\partial \Lambda_{7}^{(0)}$ and local terms [range $\left.O\left(r\left(e_{0}\right)\right)\right]$ of the order of $e^{-c r\left(e_{0}\right)}$, we obtain the basic quadratic forms in $\phi^{(0)}$ and $\psi$ :

$$
\frac{1}{2}\left\langle\phi^{(0)},\left(-\Delta_{u_{1}}+a L^{-2} Q\left(u_{1}\right)^{*} Q\left(u_{1}\right)\right) \phi^{(0)}\right\rangle+\frac{1}{2}\left\langle\Lambda_{8}^{(0) \prime} \psi, \Delta_{1, \text { loc }}^{L}\left(u_{1}\right) \Lambda_{8}^{(0)^{\prime}} \psi\right\rangle .
$$

In the small field region $\Lambda_{0}^{(0)}$ we have small block fields:

$$
\begin{gathered}
|v(p)-1| \leqq c e_{0} p\left(e_{0}\right), \quad|\psi(y)| \leqq c p\left(e_{0}\right) \lambda_{0}^{-1 / 4}, \\
\left|\left(D_{\bar{u}_{1}} \psi\right)\left(b^{\prime}\right)\right| \leqq c p\left(e_{0}\right), \quad b^{\prime} \in \Lambda_{0}^{(0) * *}
\end{gathered}
$$

where $\bar{u}_{1}\left(b^{\prime}\right)=\bar{u}_{1}\left(\left\langle b_{-}^{\prime}, b_{+}^{\prime}\right\rangle\right)$. We change nothing, then, by inserting a factor

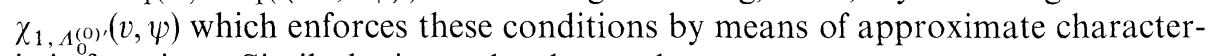
istic functions. Similarly, it can be shown that

$$
\left|A^{(0)}\right| \leqq c p\left(e_{0}\right) \quad \text { in } \quad \Lambda_{1}^{(0) *}, \quad\left|\phi^{(0)}\right| \leqq c p\left(e_{0}\right) \quad \text { in } \quad \Lambda_{1}^{(0) *}
$$

and we inset a factor $\chi_{\Lambda_{7}^{(0)}}^{\prime}$ enforcing these bounds in $\Lambda_{7}^{(0)}$.

We now consider now the interaction terms $\mathscr{P}_{0, \text { loc }}\left(\Lambda_{0}^{(0)}\right)$ and $R^{(0)}\left(u_{1}, \theta_{0} A^{(0)}\right)$, and reorganize them as follows. Vertices are restricted to $\Lambda_{8}^{(0)}$, and terms whose combined order in $e$ and $\lambda^{1 / 2}$ is greater than $\bar{n}$ are removed. The result is a standard set of terms which will appear at each iteration step. Here they are grouped into an interaction $V^{(0)}\left(\Lambda_{8}^{(0)}, u_{1}, A^{(0)}, \phi^{(0)}\right)$, a polynomial in $A^{(0)}$ and $\phi^{(0)}$. All other terms are either localized near $\Lambda_{8}^{(0) c}$ or else are of high order in couplings. The other terms are written as $\sum_{\square} W_{3}^{(0)}(\square)$, each term localized at an $r\left(e_{0}\right)$-cube, and we have a bound

$$
\left|W_{3}^{(0)}(\square)\right| \leqq e^{\bar{n} \beta}\left(\varepsilon / \varepsilon_{0}\right)^{\kappa} .
$$

Here $\beta>0$ is a fixed small power, $\kappa>d$ is a fixed large power, and $\varepsilon_{0}$ is the lattice spacing to terminate the induction.

$$
\varepsilon_{0}=\varepsilon^{\beta} \min \left\{1,\left(8 \lambda / e^{2}\right)^{1 / 2}\right\} .
$$


The bound (3.34) is sufficient for a rough treatment of such terms, since $\left(L^{k} \varepsilon / \varepsilon_{0}\right)^{\kappa}$ is summable on $k$ even with an entropy factor $\left(L^{k} \varepsilon / \varepsilon_{0}\right)^{-d}$. Terms satisfying such bounds are called irrelevant.

We avoid any further consideration of the irrelevant terms by Mayerexpanding them as in (3.19). This includes terms $W_{1}^{(0)}(\square), W_{3}^{(0)}(\square)$, as well as the small terms neglected in obtaining the quadratic forms for $A^{(0)}, f, \phi^{(0)}$, and $\psi$. Grouping all irrelevant terms localized in $\square$ into $W_{4}^{(0)}(\square)$, we write

$$
\exp \left(-\sum_{\square} W_{4}^{(0)}(\square)\right)=\sum_{S_{4}} \prod_{\square \in S_{4}}\left(\exp \left(-W_{4}^{(0)}(\square)\right)-1\right)
$$

In a similar fashion we break off the low order terms in the observables, and we obtain a sum of terms, depending on whether the relevant or irrelevant parts of $F_{k, \text { loc }}\left(X_{\sigma}\right)$ are chosen. (For observable terms, irrelevant means bounded independently of $\varepsilon$.)

We then avoid regions with irrelevant terms $\exp \left(-W_{4}^{(0)}(\square)\right)-1$ or from $F_{k \text {, loc }}\left(X_{\sigma}\right)$. Subtract from $\Lambda_{8}^{(0)}$ all such regions; call the result $\tilde{\Lambda}_{8}^{(0)}$ and define $\Lambda_{9}^{(0)}$ by deleting an $r\left(e_{0}\right)$-collar from it.

The original characteristic functions $\chi_{\Lambda_{0}^{(0)}}$ are inconvenient for our subsequent analysis because they couple block and fluctuation fields. We remove them, relying

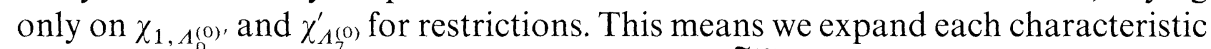
function as $\chi=1-\chi^{c}$. We obtain a sum of regions $\tilde{\Lambda}_{9}^{(0)}$ which contains only 1 -terms:

$$
\chi_{\Lambda_{9}^{(0)}}=\sum_{\tilde{\Lambda}_{9}^{(0)}} \zeta_{\tilde{\Lambda}_{9}^{\prime(0) c}}^{\prime}
$$

Here the function $\zeta_{\tilde{\Lambda}_{9}^{(0) c}}^{\prime}$ forces some field to be large $\left(\chi^{c}\right)$ in each $r\left(e_{0}\right)$-cube of $\Lambda_{9}^{(0)} \backslash \tilde{\Lambda}_{9}^{(0)}$. Then $\Lambda_{10}^{(0)}$ is defined by deleting a collar from $\tilde{\Lambda}_{9}^{(0)}$.

We now are prepared to calculate the integral over $\phi^{(0)}, A^{(0)}$ in $\Lambda_{10}^{(0)}$. We write the integrals there as normalized Gaussian integrals with conditioning at the boundary of $\Lambda_{10}^{(0)}$. This conditioning is given by $\phi^{(0)}, A^{(0)}$ in $\Lambda_{10}^{(0) c}$, and is a source of some nonlocal effects which must be dealt with. First of all the normalization factors for the Gaussian integral depend on the fields in $\Lambda_{10}^{(0) c}$. These can be written as the normalization factors without conditioning, $Z_{A_{10}^{(0)} c^{*} c}, Z_{A_{10}^{(0)}}\left(u_{1}\right)$ times quadratic forms in $\left.\left.\phi^{(0)}\right|_{\Lambda_{10}^{(0) c},} A^{(0)}\right|_{\Lambda_{10}^{(0) c} \text {. These forms are nonlocal and they must be given }}$ random walk expansions. Secondly, there are cross terms between the fields in $\Lambda_{10}^{(0)}$ and in $\Lambda_{10}^{(0) c}$ in the exponent in the normalized Gaussian integral. We take care of most of these with a translation localized near the boundary of $\Lambda_{10}^{(0)}$. The residual linear terms, of the order of $e^{-\operatorname{cr}\left(e_{0}\right)}$, are left (resulting in an uncentered Gaussian) and produce small effects in the cluster expansion.

The result is a small-field integral of the following form:

$$
\int d \mu_{\Lambda_{10}^{(0)}}^{(0)}\left(A^{(0) \prime \prime}, \phi^{(0) \prime \prime}\right) \chi_{\Lambda_{7}^{(0)}}^{\prime} \prod_{\sigma_{1}} F_{0, \text { loc }}^{\check{m}}\left(X_{\sigma_{1}}\right) \exp \left[-V^{(0)}\left(\Lambda_{8}^{(0)}, u_{1}, A^{(0)}, \phi^{(0)}\right)-\sum_{X} W_{5}^{(0)}(X)\right] .
$$

Here $A^{(0) "}, \phi^{(0) "}$ are the translated fields, and the terms $W_{5}^{(0)}(X)$ come from the random walk expansion mentioned above. The complete expression for our density is of course much more complicated; we focus on this because it is the only remaining nonlocal effect. We give a cluster expansion for it now.

Without going into details, it is worth remarking that if we pull out the terms in $V^{(0)}$ which are independent of $\phi^{(0)}, A^{(0)}$ (call these $V_{\text {const }}^{(0)}$ ), all other terms are 
uniformly small (bounded by a power of $e_{0}$ ) because of the restrictions on the fields. The cluster expansion puts the integral (3.38) in polymer form,

$$
e^{-V_{\text {const }}^{(0)}\left(\Lambda_{8}^{(0)}\right)} \sum_{\left\{X_{\alpha}\right\}} \prod_{\alpha} g_{2}\left(X_{\alpha}\right) \text {. }
$$

The polymer functions $g_{2}$ depend only on fields in $X_{\alpha}$, and exhibit exponential decay in $\left|X_{\alpha}\right|$.

The clusters $X_{\alpha}$ intersection $\Lambda_{11}^{(0) c}$ have some dependence on $\left.\phi^{(0)}\right|_{\Lambda_{10}^{(0)} c},\left.A^{(0)}\right|_{A_{10}^{(0) c} \text {. }}$ The remaining clusters have completely decoupled from the large field region. We denote the region they cover by $\Lambda_{12}^{(0)}$. In this region we resum the cluster expansion and use perturbative expansions to calculate the effective action for $v, \psi$.

The resummed integral in $\Lambda_{12}^{(0)}$ is written as

$$
z_{F}\left(\Lambda_{12}^{(0)}\right)=\frac{z_{F}\left(\Lambda_{12}^{(0)}\right)}{z\left(\Lambda_{12}^{(0)}\right)} \exp \left(\log z\left(\Lambda_{12}^{(0)}\right)\right) .
$$

The first factor is the expectation of the portion of the observable in $\Lambda_{12}^{(0)}$ in the interacting fluctuation measure. The exponent is the effective action, which is calculated as follows. We interpolate the interaction $V^{(0)}-V_{\text {const }}^{(0)}$ with a prefactor $t$. At the same time we interpolate away the characteristic functions $\chi^{\prime}$ in $\Lambda_{12}^{(0)}$. The perturbative part of the effective action is

$$
\widetilde{P}_{1}\left(\Lambda_{12}^{(0)}\right)=\sum_{\alpha=1}^{\bar{n}}-\left.\frac{1}{\alpha !} \frac{d^{\alpha}}{d t^{\alpha}} \log z_{t}\left(\Lambda_{12}^{(0)}\right)\right|_{t=0},
$$

and the remainder involves truncated expectation values in the interacting fluctuation measure with parameter $t \in[0,1]$. These truncated expectation values can be given a cluster expansion exhibiting their locality properties. Since they involve at least $\bar{n}+1$ interactions, the estimate on the resulting clusters is improved; there is a high power of couplings or a large field effect from a derivative of $\chi^{\prime}$. Thus the remainder is expressed as $\sum_{X} W_{6}^{(0)^{\prime}}(X)$, a sum of localized, irrelevant terms.

The perturbative terms involve a set of diagrams, the propagators of which are fluctuation covariances $C_{\Lambda_{12}^{(0)}}^{(0)}, C_{\Lambda_{12}^{(0)}}^{(0)}\left(u_{1}\right)$ with Dirichlet boundary conditions on $\Lambda_{12}^{(0)}$. These nonlocal covariances are replaced with our standard localized ones, $C_{\text {loc }}^{(0)}$ and $C_{\text {loc }}^{(0)}\left(u_{1}\right)$, with the difference given a random walk expansion. Any term involving a covariance other than $C_{\text {loc }}^{(0)}$ or $C_{\mathrm{loc}}^{(0)}\left(u_{1}\right)$ is extremely small, $O\left(e^{-\operatorname{cr}\left(e_{0}\right)}\right)$, and localized with an exponential decay. For simplicity we extend the range of integration of vertices to all of $\Lambda_{8}^{(0)}$; the difference involves only small, local terms in $\Lambda_{8}^{(0)} \cap \Lambda_{12}^{(0) c}$. As a result of these changes we have

$$
V_{\text {const }}^{(0)}\left(\Lambda_{8}^{(0)}\right)+\overline{\mathscr{P}}_{1}\left(\Lambda_{12}^{(0)}\right)=\mathscr{P P}_{1, \text { loc }}^{L}\left(\Lambda_{8}^{(0)}\right)+\sum_{X} W_{6}^{(0) \prime \prime}(X)
$$

A perturbative contribution to $z_{F}\left(\Lambda_{12}^{(0)}\right) / z\left(\Lambda_{12}^{(0)}\right)$ is also extracted through integration by parts. When the order in couplings is high enough, the expectation is calculated with the cluster expansion. Nonlocal covariances in the perturbative part are replaced with local ones as above.

In a final operation, we Mayer-expand the irrelevant terms $W_{6}^{(0) \prime}$ and $W_{6}^{(0) \prime \prime}$. The region $\Lambda_{13}^{(0)}$ is defined as the part of $\Lambda_{12}^{(0)}$ free of irrelevant terms, either from the 
exponent or from the observable. All the terms associated with the connected components $\left\{X_{\omega}\right\}$ of the large-field region $\Lambda_{13}^{(0) c}$ are grouped into large field functions $g_{1}\left(X_{\omega}\right)$. We rescale the $L$-lattice of blocks to unit lattice spacing. From the block field $\psi$ we get a contribution to the normalization energy:

$$
E^{(0) \prime}=(d-2)(\log L)\left|T_{1}^{(1)}\right|,
$$

and we put

$$
\mathscr{E}_{1}=\mathscr{E}_{0}+E^{(0)}+E^{(0) \prime}
$$

The result is the following expression for our density:

$$
\begin{aligned}
\varrho_{1}(v, \psi)= & \sum_{\left\{X_{\omega\}}\right\}} \int \mathscr{D} u_{\Lambda_{10}^{(0) c *}}^{(0)} \chi_{\left.1, \Lambda_{0}^{(k)}\right)} \prod_{\omega} g_{1}\left(X_{\omega}\right) \prod_{\sigma} F_{1, \operatorname{loc}}\left(X_{\sigma}\right) Z_{\Lambda_{10}^{(0) c * c}}^{(0)} Z_{\Lambda_{10}^{(0)}}^{(0)}\left(u_{1}\right) \\
& \times \exp \left[-\frac{1}{2}\left\langle\Lambda_{5}^{(0) \prime * *} f^{(1)}, \sigma_{1, \operatorname{loc}} \Lambda_{5}^{(0) \prime * *} f^{(1)}\right\rangle\right. \\
& \left.-\frac{1}{2}\left\langle\Lambda_{8}^{(0) \prime} \psi, \Delta_{1, \operatorname{loc}}\left(u_{1}\right) \Lambda_{8}^{(0) \prime} \psi\right\rangle-\mathscr{P}_{1, \operatorname{loc}}\left(\Lambda_{8}^{(0)}\right)-\mathscr{E}_{1}\right] .
\end{aligned}
$$

We write explicitly the integral over $u^{(0)}$ in $\Lambda_{10}^{(0) c *}$ because in general, normalization factors $Z_{\Lambda_{i j}^{(j)}}^{(j)}\left(u_{k}\right)$ will depend on $\left.u^{(l)}\right|_{\Lambda_{10}^{(l) c *}}$ through the background field $u_{k}$. We have also introduced the rescaled field strength $f^{(1)}(p)=\left(i e_{1}\right)^{-1} \log v(p)$. The expression (3.44) will serve as a model for our starting point for the general step.

\section{The Inductive Hypothesis}

Our starting point is an expression like (3.44) which depends on fields, $u$, $\phi$ on the unit lattice. These were the fields, $v, \psi$ on the $L$-lattice in the previous step, but we have rescaled and renamed them. We assume that we have already performed $k$ renormalization transformations and expansions of the type we are about to describe. Thus the unit lattice here corresponds to the $L^{k} \varepsilon$ lattice if we had done no rescalings. The original lattice $T_{\varepsilon}$ is now $T_{\eta}, \eta=L^{-k}$, we assume that $L_{\varepsilon}^{k}<\varepsilon_{0}$ $=\min \left\{1,\left(8 \lambda / e^{2}\right)^{1 / 2}\right\} e^{\beta}$, with $\beta>0$ small and $e \ll 1$. Thus we are stopping the inductive expansion somewhat before either of the two lengths in the problem are reached. The length 1 comes from the curvature of the scalar potential, the length $\left(8 \lambda / e^{2}\right)^{1 / 2}$ comes from the curvature of the vector field potential when $\phi$ is replaced by a value minimizing its potential. When $L^{k} \varepsilon \geqq \varepsilon_{0}$, we apply a final cluster expansion designed to exhibit the Higgs mechanisms. This will be the subject of the next paper in the series. The expected correlation length is of order $l=\max \left\{1,\left(8 \lambda / e^{2}\right)^{1 / 2}\right\}$.

Our $k$-step density has the form

$$
\begin{aligned}
& \varrho_{k}(u, \phi)=\sum_{\left\{X_{\omega}\right\}} \int \prod_{j=0}^{k-1}\left[\left.\mathscr{D} u^{(j)}\right|_{\left.\Lambda_{10}^{(j) c *}\right]} \varrho_{k}^{\prime}\left(u, \phi,\left\{X_{\omega}\right\},\left\{u^{(j)}\right\}\right),\right. \\
& \varrho_{k}^{\prime}\left(u, \phi,\left\{X_{\omega}\right\},\left\{u^{(j)}\right\}\right) \\
& =\chi_{k, \Lambda_{0}^{(k-1)},} \prod_{\omega} g_{k}\left(X_{\omega}\right) \prod_{\sigma} F_{k, \operatorname{loc}}\left(X_{\sigma}\right) \prod_{j=0}^{k-1}\left[Z_{\Lambda_{10}^{(j)}}^{(j)} Z_{\Lambda_{10}}^{(j)}\left(u_{k}\right)\right] \\
& \times \exp \left[-\frac{1}{2}\left\langle\Lambda_{5}^{(k-1) \prime * *} f^{(k)}, \sigma_{k, \text { loc }} \Lambda_{5}^{(k-1) \prime * *} f^{(k)}\right\rangle\right. \\
& \left.-\frac{1}{2}\left\langle\Lambda_{8}^{(k-1) \prime} \phi, \Lambda_{k \cdot \operatorname{loc}}\left(u_{k}\right) \Lambda_{8}^{(k-1) \prime} \phi\right\rangle-\mathscr{P}_{k, \text { loc }}\left(\Lambda_{8}^{(k-1)}\right)-\mathscr{E}_{k}\right] .
\end{aligned}
$$


If we integrate this density over the $u, \phi$ variables, we obtain our original unnormalized expectation $[F]$. The measure $d u^{(j)}$ is the normalized measure on $U(1), \int d u^{(j)}=1$.

We now explain the various elements of this formula. Each $X_{\omega}$ is a union of $r\left(e_{k-1}\right)$-cubes of the $L^{-1}$-lattice, and the $X_{\omega}$ 's do not overlap. Each $X_{\omega}$ also specifies subsets $\Lambda_{\alpha}^{(j)} \cap X_{\omega}$ for $0 \leqq j \leqq k-1,0 \leqq \alpha \leqq 13$. These are unions of $r\left(e_{j}\right)$-cubes of the $L^{j} \eta$-lattice. These sets satisfy compatibility conditions arising from our constructions. In particular, with $\Lambda_{\alpha}^{(j) c}=\bigcup_{\omega}\left(\Lambda_{\alpha}^{(j) c} \cap X_{\omega}\right)$, we have $\Lambda_{0}^{(j)} \subset \Lambda_{13}^{(j-1)}$ for $j \geqq 1$. We have covered already the case $j=0$, which is slightly different. For $j \geqq 1, \alpha=1, \ldots, 8$, 11 the sets $\Lambda_{\alpha}^{(j)}$ are determined by $\Lambda_{\alpha-1}^{(j)}$ by subtracting collar neighborhoods of width $r\left(e_{j}\right)$ in the $L^{j} \eta$-lattice. We have $\Lambda_{\alpha}^{(j)} \subset \Lambda_{\alpha-1}^{(j)}$. The sets $\Lambda_{12}^{(j)}, \Lambda_{13}^{(j)}$ need not lose anything from $\Lambda_{11}^{(j)}, \Lambda_{12}^{(j)}$, though they may be smaller. The sets $\Lambda_{9}^{(j)}, \Lambda_{10}^{(j)}$ lose a collar from $\tilde{\Lambda}_{8}^{(j)}, \tilde{\Lambda}_{9}^{(j)}$, which may be smaller than $\Lambda_{8}^{(j)}, \Lambda_{9}^{(j)}$. These sets will be defined below in a manner analogous to that in the first step. We define $\bar{\Lambda}_{\alpha}^{(j)}$ as the set in $T_{L^{-}}$ obtained as the union of $L^{j}$-blocks at the points of $\Lambda_{\alpha}^{(j)}$. The factors $g_{k}\left(X_{\omega}\right)$ represent the effect of large fields or irrelevant interactions from all previous steps. The factors $g_{k}\left(X_{\omega}\right)$ depend on $u^{(j)}, 0 \leqq j \leqq k-1$ and on $u, \phi$.

The external gauge field appearing throughout the initial density is $u_{k}$. It depends on all the $u^{(j)}$ [or equivalently, the $A^{(j)}=\left(i e_{j}\right)^{-1} \log u^{(j)}$ ]; but in $\bar{\Lambda}_{6}^{(k-1) *}$ it simplifies to

$$
u_{k}=\left(Q_{k}^{s * u} u \exp \left(-i e_{k} \eta \mathscr{D}_{k, \text { loc }} \partial^{*} Q_{k}^{e *} f^{(k)}\right)\right.
$$

where $f^{(k)}(p)=\left(i e_{k}\right)^{-1} \log u(p)$. This is just a localized version of (I.4.5.4).

The form of $u_{k}$ in $\bar{\Lambda}_{6}^{(k-1) * c}$ is quite complicated; we will see it as we construct $u_{k+1}$ in the induction step. It is important now only to know that $u_{k, b}$ depends only on the fields $u^{(j)}, u$ in a neighborhood of $b$ of size $r\left(e_{k-1}\right) / 2 L$ on $T_{1}^{(k)}$. Furthermore, the configuration is smooth in the sense that for each $j<k$ (and lattice spacing $\left.\zeta=L^{-j}\right)$, and for each $r\left(e_{j}\right)$-cube $\square$ in $\bar{\Lambda}_{1}^{(j)}$, there exists a gauge transformation $u_{k} \rightarrow u_{k}^{\lambda}$ such that

$$
u_{k, b}^{\lambda}=\exp \left(i e_{j} L^{-j} A_{b}^{\lambda}\right) \quad \text { with }\left|A_{b}^{\lambda}\right|,\left|\left(\partial^{\zeta} A^{\lambda}\right)(p)\right|,\left|\left(\partial^{\zeta *} A^{\lambda}\right)(x)\right| \leqq c p\left(e_{j}\right) r\left(e_{j}\right)
$$

in $\square$. In the $k$-th step the behavior of $u_{k}$ in $\bar{\Lambda}_{6}^{(k-1) * c}$ matters only in operations involving the Gaussian normalization factors.

The configuration $u_{k}$ on $T_{\eta}^{*}$ gives a configuration $\bar{u}_{k}$ on $T_{1}^{(k) *}$ by taking a product along the bond in $T_{1}^{(k) *}$, i.e.,

$$
\bar{u}_{k, b}=u_{k}\left(\left\langle b_{-}, b_{+}\right\rangle\right) .
$$

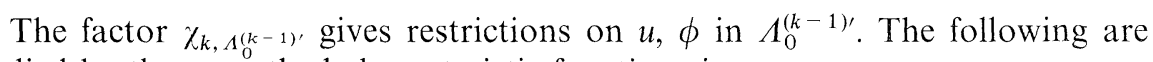
implied by the smoothed characteristic functions in $\chi_{\left.k, A_{0}^{(k-1)}\right)}$ :

$$
\begin{gathered}
\left|f^{(k)}(p)\right| \leqq c p\left(e_{k}\right), \quad p \in \Lambda_{0}^{(k-1) \prime * *}, \\
\left|\left(D_{\bar{u}_{k}} \phi\right)(b)\right| \leqq c p\left(e_{k}\right), \quad b \in \Lambda_{0}^{(k-1) \prime *}, \\
|\phi(x)| \leqq c \lambda_{k}^{-1 / 4} p\left(e_{k}\right), \quad \text { if } \quad\left(L^{k-1} \varepsilon\right)^{d}<\lambda, \quad x \in \Lambda_{0}^{(k-1)^{\prime}}, \\
||\left(\phi(x)\left|-(8 \lambda)^{-1 / 2}\left(L^{k} \varepsilon\right)^{(d-2) / 2}\right| \leqq c\left(L^{k} \varepsilon\right)^{-1} p\left(e_{k}\right), \quad \text { if } \quad\left(L^{k-1} \varepsilon\right)^{d} \geqq \lambda, \quad x \in \Lambda_{0}^{(k-1) \prime} .\right.
\end{gathered}
$$


We have incorporated some rescaling factors (powers of $L$ ) and the difference between $p\left(e_{k}\right)$ and $p\left(e_{k-1}\right)$ into the constant $c$.

The Gaussian normalization factors are given now, in a rescaled form.

$$
\begin{aligned}
Z_{\Lambda_{10}^{(j) c * c}}^{(j)}= & \int \mathscr{D} A_{\Lambda_{10}^{(j) c * c}} \delta_{\mathrm{Ax}, \Lambda_{10}^{(j)}}(A) \delta_{\left.\Lambda_{10}^{(j)}\right)^{\prime} * c}\left(Q \Lambda_{10}^{(j) c * c} A\right) \\
& \times \exp \left(-\frac{1}{2}\left\langle\Lambda_{10}^{(j) c * c} A, \partial^{*} \sigma_{j .10 c}^{L^{j} \eta} \partial \Lambda_{10}^{(j) c * c} A\right\rangle-E_{k, v}^{(j)}\left\|\Lambda_{10}^{(j) c * c}\right\|\right)
\end{aligned}
$$

where $A$ lies on the $L^{j} \eta$-lattice. The subscript to $\mathscr{D} A$ indicates where an $A$-field is integrated; the subscripts to $\delta_{\mathrm{Ax}}$ and $\delta(Q A)$ indicate which blocks have axial gauge conditions and which block bonds have conditions on $Q A$. We have Dirichlet boundary conditions in $\Lambda_{10}^{(j) c *}$. We have included a constant factor to take care of the scalings and make this independent of $k$. It is defined using

$$
\begin{gathered}
E_{k, v}^{(j)}=-\log \left[\frac{e_{j}}{2 \pi}\left(L^{j} \eta\right)^{(d-2) / 2}\right], \\
\left\|\Lambda_{10}^{(j) c * c}\right\|=\left|\Lambda_{10}^{(j) c * c}\right|-\left|\Lambda_{10}^{(j)^{\prime}}\right|\left(L^{d}-1\right)-\left|\Lambda_{10}^{(j) / c * c}\right| .
\end{gathered}
$$

Here $\left\|\Lambda_{10}^{(j) c * c}\right\|$ is the number of free integrations in $\Lambda_{10}^{(j) c * c}$ after enforcing the $\delta$-functions.

Similarly for the scalar field we have

$$
Z_{\Lambda_{10}^{(j)}}^{(j)}\left(u_{k}\right)=\int \mathscr{D} \phi_{\Lambda_{10}^{(j)}} \exp \left(-\frac{1}{2}\left\langle\Lambda_{10}^{(j)} \phi,\left(\Delta_{j, \operatorname{loc}}^{L^{J \eta}}\left(u_{k}\right)+a L^{-2} P\left(u_{k}\right)\right) \Lambda_{10}^{(j)} \phi\right\rangle-E_{k, s}^{(j)}\left|\Lambda_{10}^{(j)}\right|\right),
$$

with

$$
\begin{gathered}
P\left(u_{k}\right)=Q\left(u_{k}\right)^{*} Q\left(u_{k}\right), \\
E_{k, s}^{(j)}=-(d-2) \log L^{j} \eta .
\end{gathered}
$$

The interactions of $u, \phi$ are in $\mathscr{P}_{k, \text { loc }}\left(\Lambda_{8}^{(k-1)}\right)$. The subscript loc indicates that the terms therein couple fields no farther than $O\left(r\left(e_{k-1}\right)\right)$ apart. $\mathscr{P}_{k, \text { loc }}$ is given by a perturbation expansion up to some fixed order $\bar{n}$, which we describe in detail in a later paper. For the present analysis, it is sufficient to describe a few basic features of $\mathscr{P}_{k, \text { loc }}$.

The gauge field propagator in $\mathscr{P}_{k, \text { loc }}$ is $\mathscr{D}_{k, \text { loc }}[$ except for some renormalization transformation vertices, where it is $\sum_{j=l}^{k-1} G_{\mathrm{loc}}^{(j), \eta}$, see $\left.(2.12)\right]$ and the scalar field propagator is $G_{k, \text { loc }}\left(u_{k}\right)$. The fields $u, \phi$ appear in the diagrams through the $\eta$-lattice minimizers $u_{k}$ and

$$
\begin{aligned}
& \phi_{k}=a_{k} G_{k, \operatorname{loc}}\left(u_{k}\right) Q_{k}^{*}\left(u_{k}\right) \phi, \\
& f_{k}(p)=\left(i e_{k} \eta^{2}\right)^{-1} \log u_{k}(p) .
\end{aligned}
$$

Propagators and external fields are connected together at vertices which arise from an expansion of the $\eta$-lattice action. Vertices are restricted to $\bar{\Lambda}_{8}^{(k-1)}$; for vertices involving the gauge field the restriction is accomplished by means of a function $h_{k}$ multiplying each vector field leg at the vertex. The function $h_{k}$ changes smoothly from 0 to 1 in a neighborhood of $\bar{\Lambda}_{8}^{(k-1) * c}$. 
The dominant term for the scalar field is $P_{k}\left(\phi_{k}\right)$, where

$$
P_{k}(\phi)=\lambda_{k}|\phi|^{4}-\frac{1}{4}\left(L^{k} \varepsilon\right)^{2}|\phi|^{2}+\frac{1}{64 \lambda}\left(L^{k} \varepsilon\right)^{d} .
$$

Under the restrictions in $\chi_{k},\left|P_{k}\left(\phi_{k}\right)\right| \leqq c p\left(e_{k}\right)^{4}$. At $P_{k}$-vertices with $l$ external legs, we have its $l$-th derivative

$$
\begin{gathered}
\left|P_{k}^{(l)}\left(\phi_{k}\right)\right| \leqq c \lambda_{k}^{l / 4} p\left(e_{k}\right)^{4-l}, \text { for }\left(L^{k} \varepsilon\right)^{d} \leqq \lambda, \\
\left|P_{k}^{(l)}\left(\phi_{k}\right)\right| \leqq c\left(L^{k} \varepsilon\right)^{l} p\left(e_{k}\right)^{4-l}, \text { for }\left(L^{k} \varepsilon\right)^{d}>\lambda .
\end{gathered}
$$

In fact all terms except $P_{k}\left(\phi_{k}\right)$ in $\mathscr{P}_{k, \text { loc }}$ obey bounds $O\left(e^{\beta-\alpha}\left(L^{k} \varepsilon / \varepsilon_{0}\right)^{1 / 4-\alpha}\right)$, with $\alpha>0$, small, $\alpha<\beta$. If $\phi$ or $u$ obey better bounds, then there is a corresponding improvement in bounds on terms in $\mathscr{P}_{k, \text { loc }}$. We will prove a general theorem on estimates on perturbation expansions in a later paper.

Also in $\mathscr{P}_{k, \text { loc }}$ are vacuum energy and mass renormalization counterterms, properly localized. In $\mathscr{E}_{k}$ we keep track of normalization energies occurring over the whole lattice. This includes the basic normalization counterterm $E_{0}$, and factors from scaling and from normalization of renormalization transformations.

The observable is treated in a manner analogous to $\mathscr{P}_{k, \text { loc }}$. Each factor $F_{k, \text { loc }}\left(X_{\sigma}\right)$ is a perturbative expansion to order $\bar{m}$ of some of the factors in $F$ [those located in $X_{\sigma}$, a connected union of $r\left(e_{k-1}\right)$-cubes] with the same propagators, vertices, and external fields as before. The only difference is that the connected diagrams have at least one vertex from the observable $F\left(X_{\sigma}\right)$. Also the expansion is taken to a lower order in coupling constants for most $F$ 's. The order depends on how singular $F$ is. The sets $X_{\sigma}$ are the connected components of the smallest union of $r\left(e_{k-1}\right)$-cubes covering all vertices of all diagrams in the expansion for $F\left(\Lambda_{13}^{(k-1)}\right)$.

As in the case of the effective action, the remainders from the perturbation expansion for $F\left(X_{\sigma}\right)$ were included in the hole functional $g_{k}\left(X_{\omega}\right)$. In the case of the effective action remainder terms, this was possible because of a sufficiently high power of $e^{\beta-\alpha}\left(L^{k} \varepsilon / \varepsilon_{0}\right)^{1 / 4-\alpha}$; in the case of the observable it is possible when terms obey bounds uniform in $k$ and $\varepsilon$. The bounds may depend on the numbers of fields of various kinds in $F$, and how close they approach one another. The perturbative terms in $F\left(X_{\sigma}\right)$ are considered more carefully to show that they obey bounds independent of $\varepsilon$. Cancellations with "Wick ordering" subtractions must be performed to obtain bounds which depend only on $L^{k} \varepsilon$. For example, as long as $\left(L^{k} \varepsilon\right)^{d}<\lambda$, we expect for the expansion arising from : $|\phi(x)|^{2}$ : a bound of the order of $\left.\left(L^{k-1} \varepsilon\right)^{2}\right)^{2-d}+\lambda_{k-1}^{-1 / 2} p\left(e_{k-1}\right)^{2}$ (with the first factor replaced by $\log L^{k-1} \varepsilon$ if $d=2$ ). According to our convention, the perturbative terms in $F\left(X_{\sigma}\right)$ and $\mathscr{P}_{k, \text { loc }}$ are called "relevant" because in each case they contain insufficiently many powers of coupling constants for brute force estimation.

Finally, we assume that every factor or term in our starting expression is gauge invariant in the following senses. Gauge transformations

$$
u_{k, b} \rightarrow u_{k, b} e^{-i e_{k} \eta\left(\hat{o}^{\eta} \lambda\right)(b)}, \quad \phi(x) \rightarrow \phi(x) e^{i e_{k} \lambda(x)}
$$

leave each expression invariant. We will need to use only gauge transformations supported in $\bar{\Lambda}_{13}^{(k-1)}$, so the terms in question are scalar field forms, interaction terms in $\mathscr{P}_{k, \text { loc }}$ renormalized observables $F_{k \text {, loc }}$, characteristic functions $\chi_{k . A_{0}^{k-1}}$, 
and the normalization factors $Z_{\Lambda_{10}^{(j)}}^{(j)}\left(u_{k}\right)$. However, all expressions possess this invariance, even those that are buried in the inductive definition of $g_{k}$. Note that $\lambda$ above is any real function on $T_{\eta}$, although only its values on $T_{1}^{(k)}$ are relevant for $\phi$. We call these transformations background gauge transformations, because the integration variables $u^{(j)}, u$ are not involved. In fact, transforming these fields would affect the axial gauge conditions and the gauge field renormalization transformations. These are invariant under only a very restricted class of transformations, which we describe now.

The second kind of gauge invariance is called block field gauge invariance, and is invariance under

$$
\begin{gathered}
u_{b} \rightarrow u_{b} e^{-i e_{k}(\hat{\partial} \lambda)(b)}, \quad \phi(x) \rightarrow e^{i e_{k} \lambda(x)}, \\
u_{b}^{(j)} \rightarrow u_{b}^{(j)} \exp \left[-i e_{k} L^{j} \eta\left(\partial^{L^{j} \eta} Q_{k}^{*}-j_{j} \lambda\right)(b)\right], \quad \text { if } \quad b \in \Lambda_{1}^{(j) * c}, \\
u_{b}^{(j)} \rightarrow u_{b}^{(j)}, \quad \text { otherwise, }
\end{gathered}
$$

for $\lambda$ a function on $T_{1}^{(k)}$. Here $Q_{k}^{\prime}$ denotes the averaging operator for real-valued functions on sites. The dependence of $u_{k}$ and $u$ and the $u^{(j)}$ is such that the above transformations induce the gauge transformation $u_{k, b} \rightarrow u_{k, b} \exp \left[-i e_{k} \eta\left(\partial^{\eta} Q_{k}^{*} \lambda\right)\right]$, and thus we have invariance in the previous sense. Here, however, the variables $u$ and $u^{(j)}$ are also transformed, but in a way that does not affect the $\delta$-functions giving the axial gauge conditions and gauge field renormalization transformations. We remark that the first translation of $u^{(j)}$ in $\Lambda_{1}^{(j) *}$ accounts for the lack of a transformation there in (4.17).

In both types of gauge transformations we would have rotations of the earlier fields $\left.\phi^{(j)}\right|_{\Lambda_{10}^{(j) c}}$ which are integrated over in $g_{k}$. But since the measure $d \phi^{(j)}$ is rotationally invariant, no account need be made of these rotations.

\section{Renormalization and Decoupling in the General Step}

\subsection{Renormalization Transformation}

A density of $\varrho_{k+1}^{L}(v, \psi)$ is obtained by applying the renormalization transformations of [1] to $\varrho_{k}^{\prime}$ as follows:

$$
\begin{aligned}
\tilde{\varrho}_{k+1}^{L}(v, \psi)= & \sum_{\left\{X_{\omega}\right\}} T_{L}\left[\int \prod_{j=0}^{k-1} d u_{\Lambda_{10}^{(j) c *}}^{(j)} T_{a, L, u_{k}} \varrho_{k}^{\prime}\left(u, \phi,\left\{X_{\omega}\right\},\left\{u^{(j)}\right\}\right)\right] \\
\equiv & \sum_{\left\{X_{\omega}\right\}} \int \mathscr{D} u \delta(v / Q u) \int \prod_{j=0}^{k-1} \mathscr{D} u_{\Lambda_{10}^{(j) c *}}^{(j)} \int \mathscr{D} \phi \\
& \times \exp \left[-\frac{1}{2} a L^{-2}\left\langle\psi-Q\left(u_{k}\right) \phi, \psi-Q\left(u_{k}\right) \phi\right\rangle-E^{(k)}\right] \varrho_{k}^{\prime}\left(u, \phi,\left\{X_{\omega}\right\},\left\{u^{(j)}\right\}\right) .
\end{aligned}
$$

Here $a \approx 1$ is fixed throughout, and the normalization is

$$
E^{(k)}=-\log \left(a L^{d-2} / 2 \pi\right) .
$$

We normalize the $\delta$-function on $U(1)$ so that

$$
\int d u \delta(u) f(u)=f(1), \quad \int d u=1 .
$$


Under gauge transformations $\lambda$ of $u, \phi, u^{(j)}$ that vanish on points of $T_{L}^{(k+1)}$, we see that the $\delta$-functions and $\varrho_{k}^{\prime}$ are invariant. Since $u_{k}$ also transforms by $\lambda$, we have $Q\left(u_{k}\right) \phi$ invariant as well. Thus no change is made if we insert the axial gauge conditions

$$
\delta_{\mathrm{Ax}}(u)=\prod_{y \in T_{1}^{(k) \prime}} \prod_{x \in B(y), x \neq y} \delta\left(u\left(\Gamma_{y, x}\right)\right)
$$

into the $u$-integral above.

\subsection{Restrictions on the Fields}

We insert a partition of unity under the integrals:

$$
\begin{aligned}
1= & \sum_{P_{x} \subset \Lambda_{13}^{(k-1)^{\prime}}} \sum_{P_{y}\left(\Lambda_{13}^{(k-1)^{\prime \prime}}\right.} \sum_{P_{b}\left(\Lambda_{13}^{(k-1)^{\prime *}}\right.} \sum_{P_{p}\left(\Lambda_{13}^{(k-1)^{\prime * *}}\right.} \\
& \times \prod_{x \in P_{x}} \chi_{x}^{c} \prod_{x \in \Lambda_{13}^{(k-1)^{\prime} \backslash P_{x}}} \chi_{x} \prod_{y \in P_{y}} \chi_{y}^{c} \prod_{y \in \Lambda_{13}^{(k-1)^{\prime \prime} \backslash P_{y}}} \chi_{y} \\
& \times \prod_{b \in P_{b}} \chi_{b}^{c} \prod_{b \in \Lambda_{13}^{(k-1)^{\prime} * \backslash P_{b}}} \chi_{b} \prod_{p \in P_{p}} \chi_{p}^{c} \prod_{p \in \Lambda_{13}^{(k-1)^{\prime} * * \backslash P_{p}}} \chi_{p},
\end{aligned}
$$

where we denote

$$
\begin{aligned}
\chi_{x} & = \begin{cases}\chi\left(\lambda_{k} p\left(e_{k}\right),|\phi(x)|\right), & \text { if } \quad\left(L^{k} \varepsilon\right)^{d}<\lambda \\
\chi\left(\left(L^{k} \varepsilon\right)^{-1} p\left(e_{k}\right),|| \phi\left|-(8 \lambda)^{-1 / 2}\left(L^{k} \varepsilon\right)^{(d-2) / 2}\right|\right), & \text { if } \quad\left(L^{k} \varepsilon\right)^{d} \geqq \lambda\end{cases} \\
& =1-\chi_{x}^{c}, \\
\chi_{y} & =\chi\left(p\left(e_{k}\right),\left|\left(\psi-Q\left(u_{k}\right) \phi\right)(y)\right|\right)=1-\chi_{y}^{c}, \\
\chi_{b} & =\chi\left(p\left(e_{k}\right),\left|\left(D_{\bar{u}_{k}} \phi\right)(b)\right|\right)=1-\chi_{b}^{c}, \\
\chi_{p} & =\chi\left(e_{k} p\left(e_{k}\right),|u(p)-1|\right)=1-\chi_{p}^{c} .
\end{aligned}
$$

At each $x, y, b$, or $p$ where a $\chi^{c}$ factor is present, we expect to obtain small factors $\exp \left(-c p\left(e_{k}\right)^{2}\right) \leqq e_{k}^{\kappa}$, for any $\kappa$, using the positivity of terms in the action.

The function $\chi(p, x)$ is defined as follows: We let $\chi(1, x)$ be an even, $C^{\infty}$ function, equal to zero for $|x| \geqq 1$, and equal to one for $|x| \leqq 9 / 10$, and with

$$
\left|\frac{d^{n}}{d x^{n}} \chi(1, x)\right| \leqq c^{n} n^{c n} \text { for all } n, x \text {. }
$$

Then we put

$$
\chi(p, x)=\chi(1, x / p)
$$

The restrictions on $|\phi|$ are best understood by looking at the leading term in $\mathscr{P}_{k, \text { loc }}, P_{k}\left(\phi_{k}\right) \cong P_{k}(\phi)$, where

$$
\begin{aligned}
P_{k}(\phi) & =\lambda_{k}|\phi|^{4}-\frac{1}{4}\left(L^{k} \varepsilon\right)^{2}|\phi|^{2}+\frac{1}{64 \lambda}\left(L^{k} \varepsilon\right)^{d} \\
& =\lambda_{k}\left(|\phi|-\varrho_{0}\right)^{4}+\left(2 \lambda_{k}\right)^{1 / 2}\left(|\phi|-\varrho_{0}\right)^{3}+\frac{1}{2}\left(L^{k} \varepsilon\right)^{2}\left(|\phi|-\varrho_{0}\right)^{2}, \\
\varrho_{0} & =(8 \lambda)^{-1 / 2}\left(L^{k} \varepsilon\right)^{\frac{d-2}{2}} .
\end{aligned}
$$


For $\left(L^{k} \varepsilon\right)^{d}<\lambda$, the quartic term gets larger before the quadratic term, whereas for $\left(L^{k} \varepsilon\right)^{d}>\lambda$, the quadratic term gets large first. It is easy to see that for $|\phi(x)|$ in the support of $\chi_{x}^{c}, P_{k}(\phi(x)) \geqq O\left(p\left(e_{k}\right)^{2}\right)$.

We define the small field region $\Lambda_{0}^{(k)}$ as the union of $r\left(e_{k}\right)$-blocks, none of whose points are in $\Lambda_{13}^{(k-1) c}, P_{x}$, or in bonds, plaquettes, or blocks in $P_{b}, P_{p}, P_{y}$. The regions $\Lambda_{\alpha}^{(k)}, 0 \leqq \alpha \leqq 8$ are thus determined. We resum the partition of unity to obtain

$$
1=\sum_{\Lambda_{0}^{(k)}} \zeta_{\Lambda_{0}^{(k) c}} \chi_{\Lambda_{0}^{(k)}}
$$

where

$$
\begin{aligned}
& \zeta_{\Lambda_{0}^{(k) c}}=\sum_{P_{x}, P_{y}, P_{b}, P_{p}} \prod_{x \in P_{x}} \chi_{x}^{c} \prod_{x \in \Lambda_{13}^{(k-1)^{\prime} \backslash P_{x} \backslash \Lambda_{0}^{(k)}}} \chi_{x}
\end{aligned}
$$

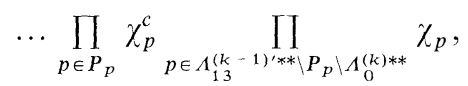

$$
\begin{aligned}
& \chi_{\Lambda_{0}^{(k)}}=\prod_{x \in \Lambda_{0}^{(k)}} \chi_{x} \ldots \prod_{p \in \Lambda_{0}^{(k) * *}} \chi_{p} .
\end{aligned}
$$

Here the sum is over subsets compatible with $\Lambda_{0}^{(k) c}, \Lambda_{13}^{(k-1)}$.

Our density now has the form

$$
\begin{aligned}
& \tilde{\varrho}_{k+1}^{L}(v, \psi)=\sum_{\left\{X_{\omega}\right\}} \sum_{\Lambda_{0}^{(k)}} \int \mathscr{D} u \mathscr{D} \phi \delta_{\mathrm{Ax}}(u) \delta(v / Q u) \int \prod_{j=0}^{k-1} \mathscr{D} u_{A_{10}^{(j)}(j)} \zeta_{\Lambda_{0}^{(k) c}} \chi_{A_{0}^{(k)}} \\
& \times \chi_{k, \Lambda_{0}^{(k-1)}} \prod_{\omega} g_{k}\left(X_{\omega}\right) \prod_{\sigma} F_{k, \operatorname{loc}}\left(X_{\sigma}\right) \prod_{j=0}^{k-1}\left[Z_{\Lambda_{10}^{(j) c * c}}^{(j)} Z_{\Lambda_{10}^{(j)}}^{(j)}\left(u_{k}\right)\right] \\
& \times \exp \left[-\frac{1}{2}\left\langle\Lambda_{5}^{(k-1) \prime * *} f^{(k)}, \sigma_{k, \operatorname{loc}} \Lambda_{5}^{\left.(k-1) * * * f^{(k)}\right\rangle}\right.\right. \\
& -\frac{1}{2} a L^{-2}\left\langle\psi-Q\left(u_{k}\right) \phi, \psi-Q\left(u_{k}\right) \phi\right\rangle \\
& \left.-\frac{1}{2}\left\langle\Lambda_{8}^{(k-1)^{\prime}} \phi, \Delta_{k, \text { loc }}\left(u_{k}\right) \Lambda_{8}^{(k-1) \prime} \phi\right\rangle-\mathscr{P}_{k, \operatorname{loc}}\left(\Lambda_{8}^{(k-1)}\right)-\mathscr{E}_{k}-E^{(k)}\right] .
\end{aligned}
$$

Let us remark that having imposed the axial gauge conditions, we resign from all but the following restricted block field gauge invariance:

$$
\begin{gathered}
\psi_{y} \rightarrow \psi_{y} e^{i e_{k} \lambda(y),} \quad \phi_{x} \rightarrow \phi_{x} e^{i e_{k}\left(Q^{\prime *} \lambda\right)(x),} \\
v_{b^{\prime}} \rightarrow v_{b^{\prime}} e^{-i e_{k} L\left(\hat{\sigma}^{I} \cdot \lambda\right)\left(b^{\prime}\right)}, \quad u_{b} \rightarrow u_{b} e^{-i e_{k}\left(\hat{\partial} Q^{\prime * \lambda}(b)\right.}, \\
u_{b}^{(j)} \rightarrow u_{b}^{(j)} \exp \left(-i e_{k} L^{j} \eta\left(\partial^{L^{\prime} \eta} Q_{k-j+1}^{*} \lambda\right)(b)\right), \quad b \in \Lambda_{1}^{(j) * c} \quad \text { only. }
\end{gathered}
$$

These transformations represent exactly the gauge invariance that was not broken by the axial gauge conditions but was broken by the renormalization transformation. By compensating with transformations of the block fields $v, \psi$, we again have an invariance. This restricted gauge invariance we intend to preserve in all subsequent operations. For example, it is easily seen that the characteristic functions we have inserted are invariant. After integrating over $\phi$, the $\phi$-rotation becomes irrelevant and we will obtain the block field invariance at the next scale, as described in the induction hypothesis.

In an analogous fashion, $\psi$ must be rotated when performing a general background gauge transformation. After integrating over $\phi$ we will obtain the invariance (4.17) at the next scale. 


\subsection{First Gauge Field Translation}

The first translation is done in $\Lambda_{1}^{(k) *}$, and it removes the $v$-field from the $\delta$-functions there. As in (3.24) we put

$$
u=u^{\prime}\left(\Lambda_{1}^{(k) *} Q^{s *} v\right),
$$

cf. also (I.6.2). (The reader may wish to refer to chapter 6 of [2], where the effects of the translations are followed without the complications of the large field regions.) Using the restrictions $|u(p)-1| \leqq e_{k} p\left(e_{k}\right)$ in $\Lambda_{0}^{(k) * *}$, and the axial gauge conditions, we obtain that

$$
u_{b}^{\prime}=e^{i e_{k} A_{b}^{\prime}} \quad \text { with } \quad\left|A_{b}^{\prime}\right| \leqq c p\left(e_{k}\right), \text { for } \quad b \in \mathcal{A}_{1}^{(k) *} .
$$

Let us define $f(p)=\left(i e_{k}\right)^{-1} \log v(p)$. The restrictions on $u(p)$ and the fact that $v=Q u$ imply that $|f(p)| \leqq c p\left(e_{k}\right)$ for $p \in \Lambda_{0}^{(k) \prime *}$. Under the translation we have

$$
f^{(k)}(p)=\Lambda_{1}^{(k) * * c}\left(i e_{k}\right)^{-1} \log u^{\prime}(p) v\left(p_{0}^{\prime}\right)+\Lambda_{1}^{(k) * *}\left(\partial A^{\prime}+L^{-2} Q^{e * f}\right)(p),
$$

where $p_{0}$ is the portion of $p$ intersecting some $B^{s}\left(b^{\prime}\right), b^{\prime} \in \Lambda_{1}^{(k) * *}$, and $p_{0}^{\prime}$ is formed by replacing each bond in $p_{0}$ with the block bond $b^{\prime}$ in $\Lambda_{1}^{(k) *}$, whose $B^{s}\left(b^{\prime}\right)$ contains it.

After this translation the background gauge field is

$$
\begin{aligned}
u_{k}= & \left(\bar{\Lambda}_{1}^{(k) * c} Q_{k+1}^{s *} v\right) \operatorname{expie_{k}} \eta\left[Q_{k}^{s *} \Lambda_{1}^{(k) *} A^{\prime}-\mathscr{D}_{k, \operatorname{loc}} \partial^{*} Q_{k}^{e *}\left(\Lambda_{1}^{(k) * *}\left(i e_{k}\right)^{-1} \log u^{\prime}(p) v\left(p_{0}^{\prime}\right)\right.\right. \\
& \left.\left.+\Lambda_{1}^{(k) * *}\left(\partial A^{\prime}+L^{-2} Q^{e * f}\right)\right)\right],
\end{aligned}
$$

for $b \in \bar{\Lambda}_{6}^{(k-1) *}$. The background field $f_{k}$ appearing at some vertices in $\mathscr{P}_{k \text {, loc }}$ and in $F_{k, \text { loc }}$ is transformed accordingly. In $\bar{\Lambda}_{2}^{(k) *}$ this simplifies to

$$
u_{k}=\left(Q_{k+1}^{s *}\right) \operatorname{expie} e_{k} \eta\left[Q_{k}^{s *} A^{\prime}-\mathscr{D}_{k, \mathrm{loc}} \partial^{*} Q_{k}^{e *}\left(\partial A^{\prime}+L^{-2} Q^{e * f}\right)\right],
$$

cf. (I.6.2.3). The quadratic form $f^{(k)}$ transforms into

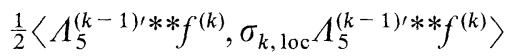

$$
\begin{aligned}
& =\frac{1}{2}\left\langle\Lambda_{5}^{(k-1) * * *} \Lambda_{1}^{(k) * * c}\left(i e_{k}\right)^{-1} \log u(p) v\left(p_{0}^{\prime}\right)\right. \\
& \left.+2 \Lambda_{1}^{(k) * *} \Lambda_{2}^{(k) c * *}\left(\partial A^{\prime}+L^{-2} Q^{e * f}\right), \sigma_{k, \operatorname{loc}} \Lambda_{5}^{(k-1) * * *} \Lambda_{1}^{(k) * * c}\left(i e_{k}\right)^{-1} \log u(p) v\left(p_{0}^{\prime}\right)\right\rangle \\
& +\frac{1}{2}\left\langle\Lambda_{1}^{(k) * *}\left(\partial A^{\prime}+L^{-2} Q^{e * f}\right), \sigma_{k, \text { loc }} \Lambda_{1}^{(k) * *}\left(\partial A^{\prime}+L^{-2} Q^{e * f}\right)\right\rangle \\
& =\mathscr{Q}_{1}+\mathscr{Q}_{1}^{\prime} \text {. }
\end{aligned}
$$

The translation affects the $\delta$-functions as follows.

$$
\delta_{\mathrm{Ax}}(u)=\delta_{\mathrm{Ac}}\left(u^{\prime}\right), \quad \delta(v / Q u)=\delta_{\Lambda_{1}^{(k) * * c}}(v / Q u) \delta_{\Lambda_{1}^{(k) * *}}\left(\frac{e_{k}}{2 \pi} Q A^{\prime}\right),
$$

where

$$
\delta_{\Lambda_{1}^{(k)^{\prime \prime}}}\left(\frac{e_{k}}{2 \pi} Q A^{\prime}\right)=\prod_{b^{\prime} \in \Lambda_{1}^{(k) \prime *}} \delta\left(\frac{e_{k}}{2 \pi}\left(Q A^{\prime}\right)\left(b^{\prime}\right)\right) .
$$

The factor $e_{k} / 2 \pi$ arises because $d u_{b}=\left(e_{k} / 2 \pi\right) d A_{b}$. 


\subsection{Gauge Transformation}

We need to make an $A^{\prime}$-dependent gauge transformation to put $u_{k}$ into a proper form. The purpose of this operation is to keep the operators $H_{k}$ appearing in $u_{k}$ and in $G_{k, \text { loc }}$ in a good gauge, i.e., Landau gauge and not axial gauge. The axial gauge operator $H_{k, \text { Ax }}$ would arise more naturally in our procedure, but it does not have the necessary regularity. properties. This operation is not performed in the first step, since $H_{0}=H_{0, \mathrm{Ax}}=I$.

The unlocalized form of the gauge transformation is based on the identity (2.20),

$$
Q_{k}^{s *} A^{\prime}-\mathscr{D}_{k} \partial^{*} Q_{k}^{e *} \partial A^{\prime}=H_{k} A^{\prime}+\partial C_{k} A^{\prime} .
$$

The operator $C_{k}$ has an exponential decay, but $C_{k}$ and $\partial C_{k}$ can have local singularities which is why the term $\partial C_{k}$ must be removed.

To do this in a way that does not introduce nonlocal dependence on $A^{\prime}$, and in a way that does not change $u_{k}$ in $\bar{\Lambda}_{3}^{(k) c *}$, we make background gauge transformations on individual terms that depend on $u_{k}$ in $\bar{\Lambda}_{3}^{(k) c * c}$. Up to some small errors, the scalar field rotations can be removed using the rotational invariance of $\mathscr{D} \phi, \mathscr{D} \psi$.

The expressions $\chi_{\Lambda_{0}^{(k)}}, \chi_{k, \Lambda_{0}^{(k-1)},}, F_{k, \text { loc }}\left(X_{\sigma}\right), Z_{\Lambda_{10}^{(j)}}^{(j)}\left(u_{k}\right),\left\langle\psi-Q\left(u_{k}\right) \phi, \psi-Q\left(u_{k}\right) \phi\right\rangle$, $\left\langle\Lambda_{8}^{(k-1)^{\prime}} \phi, \Delta_{k, \text { loc }}\left(u_{k}\right) \Lambda_{8}^{(k-1)^{\prime}} \phi\right\rangle$, and $\mathscr{P}_{k, \text { loc }}\left(\Lambda_{8}^{(k-1)}\right)$ are the ones depending on $u_{k}$ in $\bar{\Lambda}_{3}^{(k) c * c}$. The dependence is through some simple, localized expressions like

$$
\begin{gathered}
\left(\bar{\psi} Q\left(u_{k}\right) \phi\right)(y), \quad G_{k, \text { loc }}\left(u_{k} ; b_{-}, b_{+}\right) u_{k}(b), \\
\left(\bar{\phi} Q_{j}\left(u_{k}\right) G_{j, \text { loc }}\left(u_{k}\right) Q_{j}^{*}\left(u_{k}\right) \phi\right)\left(x_{1}, x_{2}\right), \quad f_{k}(p)^{4},
\end{gathered}
$$

or in similar expressions for the diagrams in $\mathscr{P}_{k, \text { loc }}$ or $F_{k, \text { loc }}$. The Gaussian normalization factors are written as in (4.9), and the dependence on $u_{k}$ is in the operators

$$
\Delta_{j, \text { loc }}\left(u_{k}\right)=a_{j} I-a_{j}^{2} Q_{j}\left(u_{k}\right) G_{j, \text { loc }}\left(u_{k}\right) Q_{j}^{*}\left(u_{k}\right) \text { and } P\left(u_{k}\right),
$$

and we have terms of the above type. However, a slightly different procedure is applied to normalization factors; we describe it later. Let us fix a set of sites where fields $\psi$ or $\phi$ sit; then the dependence on $u_{k, b}$ is only for $b$ in some cube $\square_{0}$ enclosing all points closer than $\frac{1}{2 L} r\left(e_{k-1}\right)$ to the fixed sites. $[$ There are at most some fixed number of propagators $G_{j, \text { loc }}\left(u_{k}\right)$ or $\mathscr{D}_{k, \text { loc }}$, and each has a range less than $\frac{1}{4 L} r\left(e_{k-1}\right)$. Thus we can choose $L$ such that $\square_{0}$ is a cube of size $\frac{1}{4} r\left(e_{k}\right)$. For the diagrams without external $\phi, \psi$ fields, we have to localize one vertex in a unit cube and consider the localized diagram as a separate term. We define an appropriate $\square_{0}$ containing all relevant bonds for the propagators in the localized diagram.

The gauge field appearing in any one term can be written as

$$
u_{k}=\left(Q_{k+1}^{s *} v\right) \operatorname{expie}_{k} \eta\left[\left(Q_{k}^{s *}-\mathscr{D}_{k, \text { loc }} \partial^{*} Q_{k}^{e *} \partial\right) \square A^{\prime}-L^{-2} \mathscr{D}_{k, \text { loc }} \partial^{*} Q_{k+1}^{e *} f\right]_{b},
$$

where $\square$ is a $\frac{1}{2} r\left(e_{k}\right)$-cube in $T_{1}^{(k) *}$ containing a collar neighborhood around $\square_{0}$. We extend $\square$ to a component of a $\frac{1}{2} r\left(e_{k}\right)$-neighborhood of $\Lambda_{3}^{(k) * c} \backslash \Lambda_{3}^{(k) c *}$ for all terms such that $\square_{0}$ intersects $\Lambda_{3}^{(k) * c} \backslash \Lambda_{3}^{(k) c *}$. The values of $A^{\prime}$ outside $\square$ do not matter 
because $\mathscr{D}_{k, \text { loc }}$ has a range $\frac{1}{2 L} r\left(e_{k-1}\right)$, and so $u_{k, b}$ doesn't depend on them for $b \in \square_{0}$. We now write

$$
\left(Q_{k}^{s *}-\mathscr{D}_{k, \text { loc }} \partial^{*} Q_{k}^{e *} \partial\right) \square A^{\prime}=\left(Q_{k}^{s *}-\mathscr{D}_{k} Q_{k}^{e *} \partial\right) \square A^{\prime}+w_{1}^{\prime} A^{\prime}
$$

The kernel $w_{1}^{\prime}=\left(\mathscr{D}_{k}-\mathscr{D}_{k . \text { loc }}\right) \partial^{*} Q_{k}^{*} \partial \square$ involves only the tails not included in the expansion (2.12). Using the regularity and exponential decay of $H_{j}, H_{j}$, loc along with (2.7) and scaling properties of these kernels, we find that

$$
\begin{aligned}
\left|\left(\partial w_{1}^{\prime}\right)\left(p, b^{\prime}\right)\right| & \leqq \sum_{j=1}^{k-1}\left(L^{j} \eta\right)^{-1-(d-2)-1+(d-2)} e^{-c r\left(e_{j}\right)} e^{-c \operatorname{dist}\left(p, b^{\prime}\right)} \\
& \leqq e^{-c r\left(e_{k}\right)} e^{-c \operatorname{dist}\left(p, b^{\prime}\right)}
\end{aligned}
$$

and similarly for $w_{1}^{\prime}, \partial^{*} w_{1}^{\prime}$. Also, $w_{1}^{\prime}$ is finite ranged in the sense that $w_{1}^{\prime}=w_{1}^{\prime} \square$; we use $w_{1}^{\prime}\left(b, b^{\prime}\right)$ only for $b$ in $\square_{0} \subset \square$.

The nonlocal gauge transformation (5.4.1) is now applied and we have

$$
\begin{aligned}
\left(Q_{k}^{s *}-\mathscr{D}_{k, \text { loc }} \partial^{*} Q_{k}^{e *} \partial\right) \square A^{\prime} & =\left(H_{k}+\partial C_{k}\right) \square A^{\prime}+w_{1}^{\prime} A^{\prime} \\
& =H_{k, \text { loc }} A^{\prime}+\partial C_{k} \square A^{\prime}+w_{1} A^{\prime} .
\end{aligned}
$$

We have put $w_{1}=w_{1}^{\prime}+H_{k} \square-H_{k, \text { loc }}$, and it satisfies the same bounds as $w_{1}^{\prime}$.

The background gauge transformation

$$
\begin{gathered}
u_{k, b} \rightarrow u_{k, b}^{\prime}=u_{k, b} \exp \left(-i e_{k} \eta\left(\partial \bar{\Lambda}_{3}^{(k)} C_{k} \square A^{\prime}\right)(b)\right), \\
\phi(x) \rightarrow \phi(x) \exp \left(i e_{k}\left(\bar{\Lambda}_{3}^{(k)} C_{k} \square A^{\prime}\right)(x)\right), \\
\psi(y) \rightarrow \psi(y) \exp \left(i e_{k}\left(\bar{\Lambda}_{3}^{(k)} C_{k} \square A^{\prime}\right)(y)\right),
\end{gathered}
$$

is now performed on the term localized in $\square_{0}$. The background field becomes

$$
\begin{aligned}
u_{k}^{\prime}= & \left(Q_{k+1}^{s *} v\right) \operatorname{expie}{ }_{k} \eta\left[H_{k, \operatorname{loc}} A^{\prime}+\partial \bar{\Lambda}_{3}^{(k) c} C_{k} \square A^{\prime}\right. \\
& \left.+w_{1} A^{\prime}-L^{-2} \mathscr{D}_{k, \operatorname{loc}} \partial^{*} Q_{k+1}^{e *} f\right],
\end{aligned}
$$

for $b \in \bar{\Lambda}_{3}^{(k) c * c}$; in $\bar{\Lambda}_{3}^{(k) c *}$ it is unchanged from the expression (5.3.3), obtained after the first translation. This field depends on the term considered, but we shall remove the term $w_{1} A^{\prime}$ from this expression later (only in $\bar{\Lambda}_{3}^{(k) c * c}$ ). Without $w_{1} A^{\prime}$ the field $u_{k}^{\prime}$ is independent of the term.

There are still the phase factors at $\phi$ and $\psi$. We define

$$
\phi^{\prime}(x)=\phi(x) e^{i e_{k}\left(\bar{A}_{3}^{(k)} C_{k, 10 c} A^{\prime}\right)(x)}, \quad \psi^{\prime}(y)=\psi(y) e^{i e_{k}\left(\dot{A}_{3}^{(k)} C_{k, 10 c} A^{\prime}\right)(y)} .
$$

By (2.26), $C_{k, \text { loc }}\left(x, b^{\prime}\right)$ approximates the phase factors in (5.4.5), while being independent of $\square_{0}$. The measure $d \phi$ is rotationally invariant, so we can replace $d \phi$ with $d \phi^{\prime}$ and drop the prime. We have not yet integrated over $\psi$, so a different density is obtained by replacing $\psi^{\prime}$ with $\psi$. However, the new density $\varrho_{k+1}^{L}(v, \psi)$ still has the property that $\int d v d \psi \varrho_{k+1}^{L}(v, \psi)=[F]$.

After these rotations, the scalar field still have small, term-dependent phase factors. The scalar fields appear as $\phi(x) \exp \left[i e_{k}\left(w_{2} A^{\prime}\right)(x)\right], \psi(y) \exp \left[i e_{k}\left(w_{2} A^{\prime}\right)(y)\right]$, where $w_{2}=\bar{\Lambda}_{3}^{(k)} C_{k, \text { loc }}-\bar{\Lambda}_{3}^{(k)} C_{k} \square$ satisfies a bound

$$
\left|w_{2}(x, b)\right| \leqq \exp \left(-\operatorname{cr}\left(e_{k}\right)\right) \exp (-c \operatorname{dist}(x, b))
$$


[this follows from (2.26)]. Like the $w_{1} A^{\prime}$ terms, the $w_{2} A^{\prime}$ terms will be expanded out of all expressions.

The same constructions apply to $f_{k}(p)=\left(i e_{k} \eta^{2}\right)^{-1} \log u_{k}(p)$, and we end up with $f_{k}^{\prime}(p)=\left(i e_{k} \eta^{2}\right)^{-1} \log u_{k}^{\prime}(p)$. Of course, the term $\partial \bar{\Lambda}_{3}^{(k) c} C_{k} \square A^{\prime}$ disappears. The terms involving $w_{1} A^{\prime}$ will be separated out later.

The constructions described above were motivated by a desire to preserve locality, and to avoid effects of the gauge transformation from reaching the hole functionals $g_{k}\left(X_{\omega}\right)$ or the large-field regions. The Gaussian normalization factors $Z_{\Lambda_{10}^{(j)}}^{(j)}\left(u_{k}\right)$ are intrinsically nonlocal objects; all regions are essentially tied together. Only after some expansions can some small terms be localized. Thus at this point we must resign from a local form of the gauge transformation. Recall from (5.3.4) that $u_{k}$ has been written as

$$
\left(\bar{\Lambda}_{2}^{(k) * c} u_{k}\right)\left(\bar{\Lambda}_{2}^{(k) *} Q_{k+1}^{s *} v\right) \operatorname{expie_{k}} \eta \bar{\Lambda}_{2}^{(k) *}\left(Q_{k}^{s *} A^{\prime}-\mathscr{D}_{k, \text { Ioc }} \partial^{*} Q_{k}^{e *}\left(\partial A^{\prime}+L^{-2} Q^{e * f}\right)\right)
$$

We put

$$
\begin{aligned}
& \bar{\Lambda}_{2}^{(k) *(}\left(Q_{k}^{s *}-\mathscr{D}_{k, \text { loc }} \partial^{*} Q_{k}^{e *} \partial\right) A^{\prime}=\bar{\Lambda}_{2}^{(k) *}\left(Q_{k}^{s *}-\mathscr{D}_{k, \text { loc }} \partial^{*} Q_{k}^{e *} \partial\right) \Lambda_{3}^{(k) * c} A^{\prime} \\
& +\left(Q_{k}^{s *}-\mathscr{D}_{k, \text { loc }} \partial^{*} Q_{k}^{e * \partial)} \Lambda_{3}^{(k) *} A^{\prime}=\bar{\Lambda}_{2}^{(k) *}\left(Q_{k}^{s *}-\mathscr{D}_{k, \text { loc }} \partial^{*} Q_{k}^{e *} \partial\right) \Lambda_{3}^{(k) * c} A^{\prime}\right. \\
& +H_{k, \operatorname{loc}} \Lambda_{3}^{(k) *} A^{\prime}+\partial C_{k} \Lambda_{3}^{(k) *} A^{\prime}+w_{5} A^{\prime} \text {. }
\end{aligned}
$$

Here

$$
w_{5}=\left[\left(\mathscr{D}_{k, \text { loc }}-\mathscr{D}_{k}\right) \partial^{*} Q_{k}^{e * \partial}+H_{k}-H_{k, \text { loc }}\right] \Lambda_{3}^{(k) *}
$$

is another small, exponentially decaying kernel. We can gauge away the term $\partial C_{k} \Lambda_{3}^{(k) *} A^{\prime}$, leacing us with the following background gauge field for the normalization factors:

$$
\begin{aligned}
& \left(\bar{\Lambda}_{2}^{(k) * c} u_{k}\right)\left(\bar{\Lambda}_{2}^{(k) *} Q_{k+1}^{s *} v\right) \operatorname{expie}{ }_{k} \eta\left[\bar{\Lambda}_{2}^{(k) *}\left(Q_{k}^{s *}-\mathscr{D}_{k, \text { loc }} \partial Q_{k}^{e *} \partial\right) \Lambda_{3}^{(k) * c} A^{\prime}\right. \\
& \left.-L^{-2} \bar{\Lambda}_{2}^{(k) * \mathscr{D}_{k, \text { loc }}} \partial^{*} Q_{k+1}^{e *} f+H_{k, \text { loc }} \Lambda_{3}^{(k) *} A^{\prime}+w_{5} A^{\prime}\right] \text {. }
\end{aligned}
$$

The term $w_{5} A^{\prime}$ will be removed later on; it couples $A^{\prime}$ to bonds everywhere in $T_{\eta}$.

\subsection{Second Gauge Field Translation}

We translate a second time to eliminate most of the term in $\mathscr{2}_{1}^{\prime}$ linear in $A^{\prime}$. This is analogous to what is done in Sect. I.6.1. The linear term is almost equal to $\left\langle\Lambda_{1}^{(k) * *} L^{-2} Q^{e * f}, Q_{k}^{e} \partial H_{k} A^{\prime}\right\rangle$, since by (I.6.1.5), (2.15) we have

$$
\sigma_{k} \partial A^{\prime}=Q_{k}^{e} \partial H_{k} A^{\prime}
$$

So we eliminate most of the linear term by a translation approximately equal to $\Lambda_{4}^{(k) *} L^{-2} C^{(k)} H_{k}^{*} \partial^{*} Q_{k+1}^{e *} f$.

The translation we actually use is localized, and is given by

$$
A^{\prime}=A^{(k)}-\Lambda_{4}^{(k) *} L^{-2} C_{\mathrm{loc}}^{(k)} H_{k, 1 \mathrm{loc}}^{*} \partial^{*} Q_{k+1}^{e *} f .
$$


Our construction of a $C_{\text {loc }}^{(k)}$ satisfying (2.10), (2.11) ensures that $\delta_{\mathrm{Ax}}\left(A^{\prime}\right)=\delta_{\mathrm{Ax}}\left(A^{(k)}\right)$, $\delta\left(Q A^{\prime}\right)=\delta\left(Q A^{(k)}\right)$. The quadratic form $\mathscr{Q}_{1}^{\prime}$ becomes

$$
\begin{aligned}
\mathscr{Q}_{1}^{\prime}= & \frac{1}{2}\left\langle\Lambda_{1}^{(k) * *} \partial A^{(k)}, \sigma_{k, \mathrm{loc}} \Lambda_{1}^{(k) * *} \partial A^{(k)}\right. \\
& +\left\langle\Lambda_{1}^{(k) * *} L^{-2} Q^{e *} f-\partial \Lambda_{4}^{(k) *} L^{-2} C_{\mathrm{loc}}^{(k)} H_{k, 1 \mathrm{loc}}^{*} \partial^{*} Q_{k+1}^{e *} f, \sigma_{k, 1 \mathrm{loc}} \Lambda_{1}^{(k) * *} \partial A^{(k)}\right\rangle \\
& +\frac{1}{2} L^{-4}\left\langle\Lambda_{1}^{(k) * *} Q^{e * f}-\partial \Lambda_{4}^{(k) *} C_{\mathrm{loc}}^{(k)} H_{k, 1 \mathrm{loc}}^{*} \partial^{*} Q_{k+1}^{e *} f, \sigma_{k, \mathrm{loc}}\left(\Lambda_{1}^{(k) * *} Q^{e * f}\right.\right. \\
& \left.\left.-\partial \Lambda_{4}^{(k) *} C_{\mathrm{loc}}^{(k)} H_{k, \mathrm{loc}}^{*} \partial^{*} Q_{k+1}^{e *} f\right)\right\rangle .
\end{aligned}
$$

In the second term we isolate a term localized near $\Lambda_{5}^{(k) c}$ and a small term. We write

$$
\mathscr{Z}_{2}^{\prime}=\left\langle\Lambda_{1}^{(k) * *} L^{-2} Q^{e * f}, \sigma_{k, \operatorname{loc}} \Lambda_{1}^{(k) * *} \partial \Lambda_{2}^{(k) * c} A^{(k)}\right\rangle,
$$

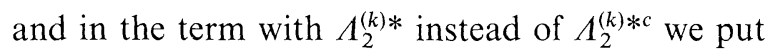

$$
\begin{aligned}
\sigma_{k, \mathrm{loc}} \partial \Lambda_{2}^{(k) *} A^{(k)} & =\sigma_{k, \mathrm{loc}} \partial \Lambda_{2}^{(k) *} \square A^{(k)}=\sigma_{k} \partial \Lambda_{2}^{(k) *} \square A^{(k)}+w_{3}^{\prime} A^{(k)} \\
& =Q_{k}^{e} \partial^{\eta} H_{k} \Lambda_{2}^{(k) *} \square A^{(k)}+w_{3}^{\prime} A^{(k)} \\
& =Q_{k}^{e} \partial^{\eta} H_{k, \text { loc }} \Lambda_{2}^{(k) *} A^{(k)}+w_{3}^{\prime \prime} A^{(k)} .
\end{aligned}
$$

The $\frac{1}{2} r\left(e_{k}\right)$-cube $\square$ is centered near the plaquette that we are evaluating $\sigma_{k, \text { loc }} \partial \Lambda_{2}^{(k) *} A^{(k)}$ at. The kernels $w_{3}^{\prime}, w_{3}^{\prime \prime}$ have range less than $\frac{1}{2} r\left(e_{k}\right)$, and we have

$$
\left|w_{3}^{\prime}(p, b)\right|, \quad\left|w_{3}^{\prime \prime}(p, b)\right| \leqq e^{-c r\left(e_{k}\right)} .
$$

In (5.5.4) we have applied our usual method for obtaining formulas for localized kernels analogous to those valid for unlocalized ones [in this case, (5.5.1)]. The precise form of the error terms will be unimportant; only bounds like (5.5.5) will matter. The $f \cdot A^{(k)}$ cross-term is now

$$
\begin{aligned}
\mathscr{Q}_{2}^{\prime} & +\left\langle\Lambda_{1}^{(k) * *} L^{-2} Q_{1}^{e *} f, w_{3}^{\prime \prime} A^{(k)}\right\rangle-\left\langle f, Q_{k+1}^{e} \partial^{\eta} H_{k, \text { loc }} C_{\text {loc }}^{(k)} L^{-2} \Lambda_{4}^{(k) *} \partial^{*} \sigma_{k, \text { loc }} \partial A^{(k)}\right\rangle \\
& +\left\langle f, L^{-2} Q_{k+1}^{e} \partial^{\eta} H_{k, \operatorname{loc}} \Lambda_{2}^{(k) *} A^{(k)}\right\rangle .
\end{aligned}
$$

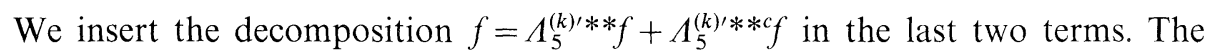
terms with $\Lambda_{5}^{(k) \prime * * c} f$ will be denoted by $\mathscr{Q}_{2}^{\prime \prime}$. The first $\Lambda_{5}^{(k) * * *}$ term involves

$$
C_{\mathrm{loc}}^{(k)} \partial^{*} \sigma_{k, \mathrm{loc}} \partial=I+w_{3}^{\prime \prime \prime}
$$

with another small, short-ranged kernel $w_{3}^{\prime \prime \prime}$. The term with the identity operator cancels the second $\Lambda_{5}^{(k) * *}$ term. Thus if we define

$$
\begin{aligned}
&\left\langle f, w_{3} A^{(k)}\right\rangle=\left\langle\Lambda_{1}^{(k) * *} L^{-2} Q_{1}^{e *} f, w_{3}^{\prime \prime} A^{(k)}\right\rangle \\
&-\left\langle\Lambda_{5}^{(k) * * *} L^{-2} f, Q_{k+1}^{e} \partial^{\eta} H_{k, \text { loc }} w_{3}^{\prime \prime \prime} A^{(k)}\right\rangle, \\
& \mathscr{Q}_{2}=\mathscr{Q}_{2}^{\prime}+\mathscr{Q}_{2}^{\prime \prime},
\end{aligned}
$$

then we have written the cross-term as $\mathscr{Q}_{2}+\left\langle f, w_{3} A^{(k)}\right\rangle$, with $\mathscr{V}_{2}$ large but localized in a $\frac{1}{2} r\left(e_{k}\right)$-neighborhood of $\Lambda_{5}^{(k) c}$, and with $w_{3}$ very small and having a range $\frac{1}{2} r\left(e_{k}\right)$.

Next we do a similar analysis on the third term in $\mathscr{Q}_{1}^{\prime}$, the term quadratic in $f$. The important contribution is when $f$ is localized in $\Lambda_{5}^{(k) * * *}$, in which case we obtain the quadratic form $\sigma_{k+1,1 \text { oc }}^{L}$ for $f$, plus small errors. The analysis here 
parallels that of [2], Sect. 6.1, with adjustments for localized kernels. Using (5.5.4), (5.5.7), and

$$
\begin{gathered}
\sigma_{k .1 \mathrm{loc}} \approx Q_{k}^{e}\left(I-\partial \mathscr{D}_{k, \mathrm{loc}} \partial^{*}\right) Q_{k}^{e *}, \\
\mathscr{D}_{k, \mathrm{loc}}+H_{k, \mathrm{loc}} C_{\mathrm{loc}}^{(k)} H_{k, \text { loc }}^{*}=\mathscr{D}_{k+1, \mathrm{loc}}^{n},
\end{gathered}
$$

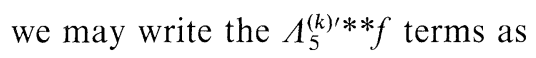

$$
\frac{1}{2}\left\langle\Lambda_{5}^{(k) * * * f,} \sigma_{k+1, \operatorname{loc}}^{L} \Lambda_{5}^{(k) * * * f\rangle}+\frac{1}{2}\left\langle f, w_{4} f\right\rangle\right.
$$

with $w_{4}$ a small, short-ranged kernel. All terms involving at least one $\Lambda_{5}^{(k) * * c} f$ are assembled into a quadratic form $\mathscr{Q}_{3}$.

To summarize the effect of the two translations, we have

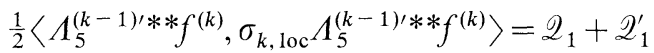

$$
\begin{aligned}
& =\mathscr{Q}_{1}+\frac{1}{2}\left\langle\Lambda_{1}^{(k) * *} \partial A^{(k)}, \sigma_{k, \operatorname{loc}} \Lambda_{1}^{(k) * * \partial} A^{(k)}\right\rangle+\frac{1}{2}\left\langle\Lambda_{5}^{(k) * * * f,} \sigma_{k+1, \operatorname{loc}}^{L} \Lambda_{5}^{(k) \prime * * f}\right\rangle \\
& +\mathscr{Q}_{2}+\mathscr{Q}_{3}+\left\langle f, w_{3} A^{(k)}\right\rangle+\frac{1}{2}\left\langle f, w_{4} f\right\rangle \text {. }
\end{aligned}
$$

Here $\mathscr{Q}_{i}, i=1,2,3$, are large linear or quadratic forms, localized near $\Lambda_{5}^{(k) c}$. They can be written as sums over the components $X_{\mu}$ of $\Lambda_{6}^{(k) c}$, i.e., $\mathscr{Q}_{i}=\sum_{\mu} \mathscr{Q}_{i}\left(X_{\mu}\right)$. The kernels $w_{3}, w_{4}$ are not localized near $\Lambda_{5}^{(k) c}$, but are small, have a range approximately $r\left(e_{k}\right)$, and become independent of the $\Lambda_{\alpha}^{(k)}$ in $\Lambda_{6}^{(k)}$, say.

After the translation, the background gauge field looks as follows. For $b \in \bar{\Lambda}_{3}^{(k) c * c},(5.4 .6)$ becomes

$$
\begin{aligned}
& u_{k}^{\prime}=\left(Q_{k+1}^{s *} v\right) \operatorname{expie}_{k} \eta\left[\left(w_{1}+H_{k, \text { loc }}\right)\left(A^{(k)}-\Lambda_{4}^{(k) *} L^{-2} C_{\text {loc }}^{(k)} H_{k, 1 \mathrm{loc}}^{*} \partial^{*} Q_{k+1}^{e *} f\right)\right. \\
& \left.+\partial \bar{\Lambda}_{3}^{(k) c} C_{k} \square A^{(k)}-L^{-2} \mathscr{D}_{k, 10 \mathrm{loc}} \partial^{*} Q_{k+1}^{e *} f\right] .
\end{aligned}
$$

In $\Lambda_{5}^{(k) *}$ we apply (5.5.11) to obtain

$$
u_{k}^{\prime}=\left(Q_{k+1}^{s *} v\right) \operatorname{expie} e_{k} \eta\left[H_{k, \text { loc }} A^{(k)}-L^{-2} \mathscr{D}_{k+1,10 \mathrm{co}}^{\eta} \partial^{*} Q_{k+1}^{e *} f+w_{1} A^{\prime}\right] .
$$

The same formula holds in $\bar{\Lambda}_{5}^{(k) *}$ for the gauge field in the normalization factors, except that we have $w_{5}$ instead of $w_{1}$.

\subsection{Expansion with Respect to the Fluctuation Field}

Let $\theta_{k}$ be a function on $T_{\eta}^{*}$ that equals 1 in $\bar{\Lambda}_{6}^{(k) *}, 0$ in $\bar{\Lambda}_{5}^{(k) * c}$, and changes smoothly from 0 to 1 in a neighborhood of $\bar{\Lambda}_{6}^{(k) *}$ of thickness $M=O(1)$. We expand most terms in our density with respect to $\theta_{k} H_{k, \text { loc }} A^{(k)}=\theta_{k} A_{k}$, and with respect to $w_{1} A^{\prime}$. This produces a number of important vertices for $A^{(k)}$, as well as irrelevant terms. We also expand in the small kernel $w_{2}$ appearing in phase factors before scalar fields. This produces only irrelevant terms. After these expansions, the background field will have the form required for the next step in $\bar{\Lambda}_{6}^{(k) *}$, with dependence on $v$ only. In the next section we consider the expansion of the normalization factors.

The new background field for the action and observables is denoted $\tilde{u}_{k+1}$, and for $b \in \bar{\Lambda}_{5}^{(k) *}$ it is given by

$$
\tilde{u}_{k+1}=\left(Q_{k+1}^{* *} v\right) \operatorname{expie}{ }_{k} \eta\left[\left(1-\theta_{k}\right) H_{k, \text { loc }} A^{(k)}-L^{-2} \mathscr{D}_{k+1,{ }_{10 c}}^{\eta} \partial^{*} Q_{k+1}^{e *} f\right] .
$$


In $\bar{\Lambda}_{6}^{(k) *}$ the $A^{(k)}$ term is absent, and except for scaling, this reduces to the form in the induction hypothesis, (4.2).

To summarize all the changes we have made on the background field, the final form of $\tilde{u}_{k+1}$ is given by (5.3.3) in $\bar{\Lambda}_{6}^{(k-1) *} \cap \bar{\Lambda}_{3}^{(k) c}$, by (5.5.13) in $\bar{\Lambda}_{3}^{(k) c * c} \cap \bar{\Lambda}_{5}^{(k) * c}$ (but without the $w_{1}$ terms), and by (5.6.1) in $\bar{\Lambda}_{5}^{(k) *}$. We will not need to define $\tilde{u}^{k+1}$ anywhere else. The corresponding background field strength is given by

$$
\widetilde{f}_{k+1}^{\eta}(p)=\left(i e_{k} \eta^{2}\right)^{-1} \log \tilde{u}_{k+1}(p) .
$$

Later we will define $u_{k+1}$ which will be slightly changed from $\tilde{u}_{k+1}$ in $\bar{\Lambda}_{2}^{(k) *} \cap \bar{\Lambda}_{4}^{(k) * c}$, but which will be defined everywhere because it appears in the normalization factors.

We need to check the regularity condition on $\tilde{u}_{k+1}$. It states that there exists a gauge transformation $\tilde{u}_{k+1} \rightarrow \tilde{u}_{k+1}^{\lambda}$ in each $r\left(e_{k}\right)$-cube $\square \subset \bar{\Lambda}_{1}^{(k)}$ such that $\tilde{u}_{k+1}^{\lambda}$ $=\exp \left(i e_{k} \eta A^{\lambda}\right)$ with $\left|A^{\lambda}\right|,\left|\partial A^{\lambda}\right|,\left|\partial^{*} A^{\lambda}\right| \leqq c p\left(e_{k}\right) r\left(e_{k}\right)$. In $\bar{\Lambda}_{1}^{(k) * c}$ we have $\tilde{u}_{k+1}=u_{k}$. so the condition follows from the induction hypothesis (4.3).

We verify the bound by first checking it for $u_{k}$, then noticing that all the operations changing $u_{k}$ into $\tilde{u}_{k+1}$ did not destroy the bound. We use the new bounds on $u(p)$ in $\bar{\Lambda}_{0}^{(k) * *}$ to estimate

$$
u_{k}=\left(Q_{k}^{s *} u\right) \exp \left[-i e_{k} \eta \mathscr{D}_{k, \text { loc }} \partial^{*} Q_{k}^{e *} f^{(k)}\right]
$$

with constants uniform in $k$. [There are bounds from the $(k-1)$-st step, but these would not yield uniform constants.] Thus we can assume that $\left|f^{(k)}(p)\right| \leqq c p\left(e_{k}\right)$ for $p \in \Lambda_{0}^{(k) * *}$.

Fix $\square \subset \bar{\Lambda}_{1}^{(k)}$ for estimating $\tilde{u}_{k+1}$. In $\square^{\prime}$ [a neighborhood of $\square$ of width $\left.\frac{1}{2} r\left(e_{k}\right)\right]$ we can write $u=\exp \left[i e_{k}(\partial \lambda+B)\right]$ with $|B(b)| \leqq c p\left(e_{k}\right) r\left(e_{k}\right)$. We have $f^{(k)}=\partial B$ in the cube, and so

$$
u_{k}=\left(Q_{k}^{s *} e^{i e_{k} \hat{\lambda} \lambda}\right) \operatorname{expie_{k}} \eta\left[\left(Q_{k}^{s *}-\mathscr{D}_{k, \text { loc }} \partial^{*} Q_{k}^{e * \partial}\right) B\right]
$$

We have used the fact that $\mathscr{D}_{k, \text { loc }}$ has range $\frac{1}{2 L} r\left(e_{k-1}\right)$. Note that $Q_{k}^{s *} e^{i e_{k} \hat{c} \lambda}$ is a gauge transformation (generated by $Q_{k}^{\prime *} \lambda$ ), so we can delete it from $u_{k}$.

Our desired bound now follows because by (5.4.4),

$$
\left(Q_{k}^{s *}-\mathscr{D}_{k, \mathrm{loc}} \partial^{*} Q_{k}^{e *} \partial\right) \square^{\prime} B=\left(H_{k, \text { loc }}+\hat{\partial} C_{k}+w_{1}\right) \square \square^{\prime} B .
$$

The kernels $H_{k, \text { loc }}, w_{1}$ and their derivatives are bounded, so $A^{\lambda}, \partial A^{\lambda}, \partial^{*} A^{\lambda}$ are finally all bounded by $c p\left(e_{k}\right) r\left(e_{k}\right)$.

The first operation we performed was a translation, which of course does not spoil the regularity of $u_{k}$. We then made a gauge transformation and removed the small kernel $w_{1}$. The gauge transformation does not change the regularity, and $\partial w_{1}, \partial^{*} w_{1}$ are small, so the bounds remain valid. After another translation we removed the field $\theta_{k} H_{k, \text { loc }} A^{(k)}$. This field satisfies $\partial\left(\theta_{k} H_{k, \text { loc }} A^{(k)}\right) \leqq c p\left(e_{k}\right)$ because $A^{(k)}$ $\leqq c p\left(e_{k}\right)$ and because $\partial H_{k, \text { loc }}, H_{k, \text { loc }}$, and derivatives of $\theta_{k}$ are bounded. Similarly $\partial^{*}\left(\theta_{k} H_{k, \text { loc }} A^{(k)}\right), \theta_{k} H_{k, \text { loc }} A^{(k)}$ are bounded by $c p\left(e_{k}\right)$. Thus removing $\theta_{k} H_{k, \text { loc }} A^{(k)}$ does not spoil the regularity, and $\tilde{u}_{k+1}$ satisfies the regularity condition. In an analogous fashion we can check that the $j$-th regularity condition for $r\left(e_{k}\right)$-cubes remains valid. 
We now proceed with the expansions. We do not expand in the characteristic functions $\chi_{\Lambda_{0}^{(k)}}, \chi_{k, \Lambda_{0}^{(k+1)}}$. We take terms which came from the original expressions $F_{k, \text { loc }}$,

$$
\left\langle\psi-Q\left(u_{k}\right) \phi, \psi-Q\left(u_{k}\right) \phi\right\rangle, \quad\left\langle\Lambda_{8}^{(k-1)^{\prime}} \phi, \Lambda_{k, \operatorname{loc}}\left(u_{k}\right) \Lambda_{8}^{(k-1)^{\prime}} \phi\right\rangle,
$$

and $\mathscr{P}_{k, \text { loc }}\left(\Lambda_{8}^{(h-1)}\right)$, and write the background field as

$$
\tilde{u}_{k+1} \tilde{u}=\tilde{u}_{k+1} \operatorname{expie}_{k} \eta\left[\theta_{k} H_{k, \text { loc }} A^{(k)}+w_{1} A^{\prime}\right]=\tilde{u}_{k+1} e^{i e_{k} \eta \tilde{A}} .
$$

The scalar fields appear with factors $e^{i e_{k} w_{2} A^{\prime}}$ before then. All expressions depend on $A^{(k)}, f$ only locally, or at most within a component of $\Lambda_{4}^{(k) c}$.

The first expansion we give is for $\tilde{u}_{k+1} \tilde{u}$ itself. (We derive some expansions for the $j$-th step objects for use in the next section. The expansions are modeled after ones in [7], so we will be brief.]

We have with $\zeta=L^{-j}, \tilde{A}$ scaled to the $\zeta$-lattice,

$$
\tilde{u}_{k+1} \tilde{u}=\tilde{u}_{k+1}\left(1+\sum_{n=1}^{\infty}\left(i e_{j} \zeta \tilde{A}\right)^{n} / n !\right)=\tilde{u}_{k+1}\left(1+F_{1, j}(\tilde{A})\right) .
$$

Next we expand $Q_{l}\left(\tilde{u}_{k+1} \tilde{u}\right), l=1$ or $j, j<k$,

$$
\begin{aligned}
\left(Q_{l}\left(\tilde{u}_{k+1} \tilde{u}\right) \phi\right)(y) & =\sum_{x \in B_{l}(y)} L^{-l d} \tilde{u}_{k+1}\left(\Gamma_{y, x}^{(l)}\right) \phi(x)\left(1+\sum_{n=1}^{\infty}\left(i e_{j} \zeta A\left(\Gamma_{y, x}^{(l)}\right)\right)^{n} / n !\right) \\
& =\left(Q_{l}\left(\tilde{u}_{k+1}\right) \phi\right)(y)+\left(F_{2, l}\left(\tilde{A}, \tilde{u}_{k+1}\right) \phi\right)(y) .
\end{aligned}
$$

Inserting this formula into $\left|\left(\psi-Q\left(\tilde{u}_{k+1} \tilde{u}\right) \phi\right)(y)\right|^{2}$, we obtain the vertices new to this step. For the covariant derivative on the $\zeta$-lattice, we have

$$
\left(\mathscr{D}_{\tilde{u}_{k+1} \tilde{u}} \phi\right)(b)=\left(D_{\tilde{u}_{k+1}} \phi\right)(b)+\tilde{u}_{k+1, b} F_{1, j}(\tilde{A}) \phi\left(b_{+}\right) .
$$

For the basic quadratic form with Neumann boundary conditions on $\Omega$ giving rise to $G_{j}(\Omega)$, we have

$$
-\Delta_{\tilde{u}_{k+1} \tilde{u}, \Omega}^{N}+a_{j} P_{j}\left(\tilde{u}_{k+1} \tilde{u}\right)=-\Delta_{\tilde{u}_{k+1}, \Omega}^{N}+a_{j} P_{j}\left(\tilde{u}_{k+1}\right)-V_{j}(\Omega),
$$

where $V_{j}(\Omega)$ is obtained by inserting (5.6.8), (5.6.9) into the left-hand side. This leads to an expansion of the scalar field propagator in a fixed region $\Omega$ :

$$
G_{j}\left(\Omega, \tilde{u}_{k+1} \tilde{u}\right)=G_{j}\left(\Omega, \tilde{u}_{k+1}\right)+G_{j}\left(\Omega, \tilde{u}_{k+1}\right) V_{j} G_{j}\left(\Omega, \tilde{u}_{k+1} \tilde{u}\right) .
$$

The terms in $V_{j}$ are small $\left(O\left(e_{j}^{1-\alpha}\right)\right)$, bounded kernels, either alone or applied to $D_{\tilde{u}_{k+1}}$ or $D_{\tilde{u}_{k+1}}^{*}$. Thus the regularity properties of $G_{j}\left(\tilde{u}_{k+1}\right), D_{\tilde{u}_{k+1}} G_{j}\left(\tilde{u}_{k+1}\right)$ imply that we can develop this expansion to any order.

We insert this into $G_{j, \text { loc }}\left(\tilde{u}_{k+1} \tilde{u}\right)$ to obtain

$$
\begin{aligned}
G_{j, \text { loc }}\left(\tilde{u}_{k+1} \tilde{u}\right)\left(x_{1}, x_{2}\right)= & G_{j, \text { loc }}\left(\tilde{u}_{k+1}\right)\left(x_{1}, x_{2}\right) \\
& +\zeta_{j}^{\prime \prime}\left(x_{1}, x_{2}\right) \sum_{\alpha} \lambda_{\alpha}\left(G_{j}\left(\square_{\alpha}, \tilde{u}_{k+1}\right) V_{j} G_{j}\left(\square_{\alpha}, \tilde{u}_{k+1} \tilde{u}\right)\right)\left(x_{1}, x_{2}\right) .
\end{aligned}
$$

The second term can be changed slightly by changing the set $\square_{\alpha}$ in $G_{j}$ and changing the tails of the operators. The difference is $w_{6}^{\prime}$, a small $\left(O\left(e^{-\operatorname{cr}\left(e_{j}\right)}\right)\right)$, local kernel with small covariant derivatives, and depending only locally on $\tilde{u}_{k+1}, \tilde{u}$. We obtain

$$
G_{j, \operatorname{loc}}\left(\tilde{u}_{k+1} \tilde{u}\right)=G_{j, \text { loc }}\left(\tilde{u}_{k+1}\right)+G_{j, \operatorname{loc}}\left(\tilde{u}_{k+1}\right) V_{j} G_{j, 1 \mathrm{loc}}\left(\tilde{u}_{k+1} \tilde{u}\right)+w_{6}^{\prime} .
$$


This is now iterated to yield

$$
\begin{aligned}
G_{j, \text { loc }}\left(\tilde{u}_{k+1} \tilde{u}\right)= & \sum_{n=0}^{\bar{n}} G_{j, \text { loc }}\left(\tilde{u}_{k+1}\right)\left[V_{j} G_{j, \text { loc }}\left(\tilde{u}_{k+1}\right)\right]^{n} \\
& +G_{j, \text { loc }}\left(\tilde{u}_{k+1}\right)\left[V_{j} G_{j, \text { loc }}\left(\tilde{u}_{k+1}\right)\right]^{\bar{n}} V_{j} G_{j, \text { loc }}\left(\tilde{u}_{k+1} \tilde{u}\right)+w_{6},
\end{aligned}
$$

with another small kernel $w_{6}$.

These expansions are inserted in $F_{k, \text { loc }},\left\langle\psi-Q\left(u_{k}\right) \phi, \psi-Q\left(u_{k}\right) \phi\right\rangle, \mathscr{P}_{k, \text { loc }}$, and in

$$
\Delta_{k, \text { loc }}\left(\tilde{u}_{k+1} \tilde{u}\right)=a_{k} I-a_{k}^{2} Q_{k}\left(\tilde{u}_{k+1} \tilde{u}\right) G_{k, \operatorname{loc}}\left(\tilde{u}_{k+1} \tilde{u}\right) Q_{k}^{*}\left(\tilde{u}_{k+1} \tilde{u}\right) .
$$

We expand the phase factors as $1+\left(e^{i e_{k} w_{2} A^{\prime}}-1\right)$, the second term being extremely small. We also have the field strength expanded as

$$
f_{k}^{\prime}=\widetilde{f}_{k+1}^{\eta}+\partial w_{1} A^{\prime}+\partial\left(\theta_{k} H_{k, \text { loc }} A^{(k)}\right) \text {. }
$$

Any term involving $w_{1}$ or $w_{2}$, and terms of higher than $\bar{n}$-th order in $e_{k}$ are irrelevant and will be treated separately. The lower order terms are polynomials in $A^{(k)}$. All terms are local.

Let us summarize these expansions as follows. In the action we have written

$$
\begin{aligned}
\frac{1}{2} a L^{-2}\left\langle\tilde{\psi}-Q\left(u_{k}^{\prime}\right) \tilde{\phi}, \tilde{\psi}-Q\left(u_{k}^{\prime}\right) \tilde{\phi}\right\rangle \\
\quad+\frac{1}{2}\left\langle\Lambda_{8}^{(k-1)^{\prime}} \tilde{\phi}, \Delta_{k, \operatorname{loc}}\left(u_{k}^{\prime}\right) \Lambda_{8}^{(k-1)^{\prime}} \tilde{\phi}\right\rangle+\mathscr{P}_{k, \operatorname{loc}}\left(\Lambda_{8}^{(k-1)} \tilde{\phi}, u_{k}^{\prime}\right) \\
=\frac{1}{2} a L^{-2}\left\langle\psi-Q\left(\tilde{u}_{k+1}\right) \phi, \psi-Q\left(\tilde{u}_{k+1}\right) \phi\right\rangle+\frac{1}{2}\left\langle\Lambda_{8}^{(k-1)^{\prime}} \phi, \Lambda_{k, \text { loc }}\left(\tilde{u}_{k+1}\right) \Lambda_{8}^{(k-1)^{\prime}} \phi\right\rangle \\
\quad+\mathscr{P}_{k, \text { loc }}\left(\Lambda_{8}^{(k-1)}, \phi, \tilde{u}_{k+1}\right)+R^{(k)}\left(u_{k+1}, \theta_{k} H_{k, \text { loc }} A^{(k)}\right)+\sum_{\square} W_{1}^{(k)}(\square) .
\end{aligned}
$$

The tildes on $\phi$ and $\psi$ indicate the presence of the phase factors. Here $W_{1}^{(k)}(\square)$ is localized near $\square$, an $r\left(e_{k}\right)$-cube in $\Lambda_{2}^{(k)}$, and $\left|W_{1}^{(k)}(\square)\right| \leqq e_{k}^{\bar{n}-1-\alpha .}$ (Two powers of $e_{k}$ may be needed to beat the bounds on $\phi$.) If we define

$$
\begin{aligned}
& \widetilde{R}^{(k)}\left(\tilde{u}_{k+1}, \theta_{k} H_{k, \text { loc }} A^{(k)}\right) \\
& =\sum_{n=1}^{n}\left[\frac { d ^ { n } } { d e ^ { \prime n } } \left(\frac{1}{2} a L^{-2}\left\|\psi-Q\left(\tilde{u}_{k+1} e^{i e^{\prime} e_{k} \eta \theta_{k} H_{k, 10 c} A^{(k)}}\right) \phi\right\|^{2}\right.\right. \\
& \left.\left.\quad+\frac{1}{2}\left\langle\Lambda_{8}^{(k-1) \prime} \phi, \Lambda_{k, \operatorname{loc}}(\ldots) \Lambda_{8}^{(k-1)^{\prime}} \phi\right\rangle+\mathscr{P}_{k, \operatorname{loc}}\left(\Lambda_{8}^{(k-1)}, \phi, \ldots\right)\right)\right]_{e^{\prime}=0}
\end{aligned}
$$

then $R^{(k)}$ can be obtained by replacing propagators $G_{k}\left(\square, \tilde{u}_{k+1}\right)$ with $G_{k, \text { loc }}\left(\tilde{u}_{k+1}\right)$ and eliminating extra kernels $\zeta_{k}^{\prime \prime}$ explicitly (not in $G_{k, \text { loc }}\left(\tilde{u}_{k+1}\right)$ ).

In a similar fashion we put

$$
F_{k, \text { loc }}\left(X_{\sigma}\right)=F_{k, \text { loc }}^{(\bar{m})}\left(X_{\sigma}\right)+\tilde{F}_{k, \text { loc }}\left(X_{\sigma}\right)
$$

where $F_{k, \text { loc }}^{(\bar{m})}\left(X_{\sigma}\right)$ is defined by replacing $G_{k}\left(\square, \tilde{u}_{k+1}\right), \zeta_{k}^{\prime \prime}$ in

$$
\sum_{m=0}^{\bar{m}}\left[\frac{d^{m}}{d e^{\prime m}} F_{k, \text { loc }}\left(X_{\sigma}, \tilde{u}_{k+1} e^{i e^{\prime} e_{k} \eta \theta_{k} H_{k, 10 \mathrm{loc}} A^{(k)}}\right)\right]_{e^{\prime}=0} .
$$

All remainder terms are in $\widetilde{F}_{k, \text { loc }}\left(X_{\sigma}\right)$, and we have $\left|\widetilde{F}_{k, \text { loc }}\left(X_{\sigma}\right)\right| \leqq c(F)$. 


\subsection{The Gaussian Normalization Factors}

The expansion with respect to the fluctuation field $A^{(k)}$ must be performed with special care in the Gaussian normalization factors. Nonlocal terms naturally arise, which must then be organized properly and treated with random walk expansions. At this point, the background field in the normalization factors is given by (5.4.10), which simplifies to (5.5.14) in $\bar{\Lambda}_{5}^{(k) *}$. We express it as $\mathcal{u}_{k+1} \tilde{\tilde{u}}$, with

$$
\tilde{\tilde{u}}=\exp i e_{k} \eta\left[\theta_{k} H_{k, \text { loc }} A^{(k)}+w_{5} A^{\prime}\right] \equiv \operatorname{expie_{k}} \eta \tilde{\tilde{A}} \text {. }
$$

In this way the field $u_{k+1}$ is defined, and after $\tilde{A}$ is expanded away, it remains in the normalization factors for the next renormalization transformation. One could apply (5.4:10) inductively to obtain a complete formula for $u_{k+1}$ on the whole lattice. We will only need to use the fact that it agrees with $\tilde{u}_{k+1}$ in $\bar{\Lambda}_{5}^{(k) *}$, cf. (5.6.1). The regularity conditions can be checked for $u_{k+1}$ in the same manner as for $\tilde{\tilde{u}}_{k+1}$.

In the integral representation for the normalization factor $Z_{A_{10}^{(j)}\left(u_{k}\right)}^{\left(u_{k}\right)}$, rescaled to the unit lattice, we have the quadratic form (with Dirichlet boundary conditions)

$$
\begin{aligned}
C_{\Lambda_{10}^{(j)}(j)}^{(j)}\left(u_{k+1} \tilde{\tilde{u}}\right)^{-1} & =\Delta_{j, 10 \mathrm{loc}}\left(u_{k+1} \tilde{\tilde{u}}\right)+a L^{-2} P\left(u_{k+1} \tilde{\tilde{u}}\right) \\
& =\Delta_{j, 10 c}\left(u_{k+1}\right)+a L^{-2} P\left(u_{k+1}\right)-W^{(j)} \\
& =C_{\Lambda_{10}^{(j)}(j)}^{(j)}\left(u_{k+1}\right)^{-1}-W^{(j)} .
\end{aligned}
$$

All the terms from our expansions of the last section for $\Delta_{j, \text { loc }}, P$, with $\tilde{u}_{k+1}$ replaced with $u_{k+1}, \tilde{u}$ replaced with $\tilde{\tilde{u}}$, are included in $W^{(j)}$. Thus we have

and so

$$
\begin{aligned}
C_{\Lambda_{10}^{(j)}}^{(j)}\left(u_{k+1} \tilde{\tilde{u}}\right)^{-1}= & \left.C_{\Lambda_{10}^{(j)}\left(u_{k+1}\right.}^{(j)}\right)^{-1 / 2}\left(I-C_{\Lambda_{10}^{(j)}}^{(j)}\left(u_{k+1}\right)^{1 / 2} W^{(j)}\right. \\
& \left.\times C_{\Lambda_{10}^{(j)}}^{(j)}\left(u_{k+1}\right)^{1 / 2}\right) C_{\Lambda_{10}^{(j)}}^{(j)}\left(u_{k+1}\right)^{-1 / 2},
\end{aligned}
$$

$$
Z_{\Lambda_{10}^{(j)}}^{(j)}\left(u_{k}\right)=Z_{\Lambda_{10}^{(j)}}^{(j)}\left(u_{k+1}\right)\left[\operatorname{det}\left(I-C_{\Lambda_{10}^{(j)}}^{(j)}\left(u_{k+1}\right)^{1 / 2} W^{[j)} C_{\Lambda_{10}^{(j)}}^{(j)}\left(u_{k+1}\right)^{1 / 2}\right]^{-1 / 2} .\right.
$$

Each term in $W^{(j)}$ has at least one factor $e_{j}$, and all fields are logarithmically bounded. Thus the operator after the identity is bounded by a very small number. Thus the determinant can be expanded as

$$
\exp \left[\sum_{l=1}^{\infty} \frac{1}{2 l} \operatorname{tr}\left(C_{\Lambda_{10}^{(j)}}^{(j)}\left(u_{k+1}\right) W^{(j)}\right)^{l}\right] .
$$

The operator $C_{\left.A_{1, j}\right)}^{(j)}\left(u_{k+1}\right)$ is our first encounter with nonlocal effects. To treat it we apply the generalized random walk expansion (2.45), modified slightly to use cubes of size $L^{k-j} r\left(e_{k}\right)$ in $T_{L^{-j}}$. We need a similar expansion for $W^{(j)}$ into terms defined in regions $X$ with appropriate decay estimates.

For example, we have in $F_{1, j}\left(\tilde{\tilde{A}}_{b}\right)$ a series involving powers of $\left(w_{5} A^{\prime}\right)_{b}$, with a nonlocal kernel $w_{5}$. We put these powers in the form of a sum on $X$ of quantities defined in $X$ only. To each $b \in T_{\eta}$ and each collection of bonds $b_{1}, \ldots, b_{m} \in T^{(k)}$ we associate in some arbitrary manner a set $X$ (a connected union of $r\left(e_{k}\right)$-cubes containing them). Then we put

$$
\left.\left.w_{b, m}(X)=\sum_{\substack{\left(b_{1}, \ldots b_{m}\right) \\ \text { compatible with } b, X}} \prod_{l=1}^{m}\left(w_{5} A^{\prime}\right)_{b}^{m}=\sum_{X} w_{b, m}(X), b_{l}\right) A^{\prime}\left(b_{l}\right)\right),
$$


where compatible means that $b_{1}, \ldots, b_{m}, b$ were associated to $X$ as above. We have $\left|A^{\prime}\left(b_{l}\right)\right| \leqq c p\left(e_{k}\right),\left|w_{5}\left(b, b_{l}\right)\right| \leqq e^{-c r\left(e_{k}\right)} e^{-c \operatorname{dist}\left(b . b_{l}\right)}$ and so

$$
\left|w_{b, m}(X)\right| \leqq e^{-\operatorname{cr}\left(e_{k}\right)|X|} .
$$

We may use (5.7.5) to analyze the interaction terms generated in the expansion with respect to $\tilde{\widetilde{A}}=\theta_{k} H_{k, \text { loc }} A^{(k)}+w_{5} A^{\prime}$. Treating for the moment only the high order terms, we obtain an expansion for $F_{1, j}$ in (5.6.7):

$$
F_{1, j}\left(\tilde{\tilde{A}}_{\zeta}^{\zeta}\right)=\sum_{n=1}^{\bar{n}} \zeta^{-1}\left(i e_{j} \zeta \tilde{\tilde{A}}_{b}^{\zeta}\right)^{n} / n !+\sum_{X} F_{1, j . b}(X)
$$

with $\left|F_{1, j, b}(X)\right| \leqq e_{j}^{\bar{n}+1-\alpha} e^{-\operatorname{cr}\left(e_{k}\right)|X|^{-}}$. (Here $|X|^{-}$is defined as $\max \{0,|X|-1\}$, and $\tilde{\tilde{A}}$ has been rescaled to the $\zeta=L^{-i}$ lattice.) In a similar fashion we can write

$$
\begin{aligned}
\left(F_{2, j}\left(\tilde{\widetilde{A}}^{\zeta}, u_{k+1}\right) \phi\right)(y)= & \sum_{x \in B_{j}(y)} \zeta^{d} u_{k+1}\left(\Gamma_{y, x}^{(j)}\right) \phi(x) \\
& \times \sum_{n=1}^{\bar{n}}\left(i e_{j} \zeta \tilde{A}\left(\Gamma_{y, x}^{(j)}\right)\right)^{n} / n !+\sum_{X}\left(F_{2, j}(X) \phi\right)(y),
\end{aligned}
$$

with

$$
\left|F_{2, j}(X ; y, x)\right| \leqq \zeta^{d} e_{j}^{\bar{n}+1-\alpha} e^{-\operatorname{cr}\left(e_{k}\right)|X|^{-}} .
$$

These expansions can be inserted into $V_{j}$, yielding

$$
V_{j}=V_{j}^{(\bar{n})}+\sum_{X} V_{j}(X)
$$

the first term containing the expansions to order $\bar{n}$ in $e_{j}$, the second containing the remaining terms. Both terms involve small, bounded kernels (of order $e_{j}^{1-\alpha}$ for $V^{(\bar{n})}$, of order $e_{j}^{\bar{n}+1-\alpha} e^{-\operatorname{cr}\left(e_{k}\right)|X|^{-}}$for $\left.V(X)\right)$, alone or applied to $D_{u_{k+1}}$ or $D_{u_{k+1}}^{*}$.

Next we examine the propagators, and expand in $V_{j}$ to all orders:

$$
G_{j}\left(\Omega, u_{k+1} \tilde{\tilde{u}}\right)=G_{j}\left(\Omega, u_{k+1}\right)+\sum_{n=1}^{\infty} G_{j}\left(\Omega, u_{k+1}\right)\left[V_{j} G_{j}\left(\Omega, u_{k+1}\right)\right]^{n} .
$$

Thus we have

$$
\begin{aligned}
\mathrm{G}_{j, \text { loc }}\left(u_{k-1} \tilde{\tilde{u}}\right)\left(x_{1}, x_{2}\right)= & G_{j .10 c}\left(u_{k+1}\right)\left(x_{1}, x_{2}\right)+\zeta_{j}^{\prime \prime}\left(x_{1}, x_{2}\right) \sum_{n=1}^{\infty}\left(G_{j}\left(\square\left(x_{1}, x_{2}\right), u_{k+1}\right)\right. \\
& \left.\times\left[V_{j} G_{j}\left(\square\left(x_{1}, x_{2}\right), u_{k+1}\right)\right]^{n}\right)\left(x_{1}, x_{2}\right) .
\end{aligned}
$$

We insert the expansion for $V_{j}$ into this formula, and insert expansions for $Q_{j}\left(u_{k+1} \tilde{\tilde{u}}\right), Q\left(u_{k+1} \tilde{\tilde{u}}\right), G_{j, 1 \text { loc }}\left(u_{k+1} \tilde{\tilde{u}}\right)$ into $\Delta_{j, \text { loc }}\left(u_{k+1} \tilde{\tilde{u}}\right)+a L^{-2} P\left(u_{k+1} \tilde{\tilde{u}}\right)$. Terms whose order in $e_{j}$ (or equivalently in $\tilde{A}$ ) is between 1 and $\bar{n}$ are considered as part of $-W^{(j, \bar{n})}$. Terms of higher order, or involving $F_{2, j}(X)$ or $V_{j}(X)$ are grouped into an expansion $\sum_{X}-W^{(j)}(X)$, with $\left|W^{(j)}\left(X ; x_{1}, x_{2}\right)\right| \leqq e_{j}^{\bar{n}+1-\alpha} e^{-\operatorname{cr}\left(e_{k}\right)|X|^{-}}$. Thus we have written the interaction term in (5.7.2) as

$$
W^{(j)}=W^{(j, \bar{n})}+\sum_{X} W^{(j)}(X)
$$

The lower order terms need to be resummed by gathering terms with different $j$ into a perturbative expression. This is because $e^{-c r\left(e_{k}\right)}$ is not small enough to 
compensate for having only a few powers of $e_{j}$. We must replace $G_{j}\left(\square\left(x_{1}, x_{2}\right), u_{k+1}\right)$ with $G_{j}\left(\Omega, u_{k+1}\right)$ for some fixed $\Omega$. This is accomplished with a random walk expansion for $G_{J}(\Omega, u)$. Such an expansion is given in [6]. It takes the form

$$
G_{j}(\Omega, u)=\sum_{\omega} G(\omega)
$$

where $\omega$ is a walk on the lattice of $M$-cubes in $T_{L}$, . Each $G(\omega)$ has regularity as in (2.30), as well as an exponential decay in the length of the walk. By summing over an appropriate subset of walks that remain inside $X$, a union of $L^{k-j} r\left(e_{k}\right)$-cubes, we obtain $G_{j}(\Omega, X, u)$. [An analogous construction for $C_{A}^{(k)}(u)$ is described in (2.42) -(2.45). $]$ Walks that stay within $\square\left(x_{1}, x_{2}\right) \cap \Omega$ define $G_{j, \text { loc }}\left(\Omega, u ; x_{1}, x_{2}\right)$, which is then nonzero only if $\left|x_{1}-x_{2}\right| \leqq O\left(L^{k-j} r\left(e_{k}\right)\right)$. A convex combination as in (2.27) is used to preserve regularity across boundaries of $M$-cubes. The result is the expansion

$$
G_{j}(\Omega, u)=G_{j, \text { loc }}(\Omega, u)+\sum_{X} G_{j}(\Omega, X, u) .
$$

Of course, $G_{j}\left(\Omega, X, u ; x_{1}, x_{2}\right)=0$ unless both $x_{1}$ and $x_{2}$ are in $X$. All operators obey the usual regularity bounds, provided $\operatorname{dist}\left(\left\{x_{1}, x_{2}\right\}, \Omega^{c}\right)>c$. The bound on $G_{j}(\Omega, X, u)$ has in addition a factor $e^{-\operatorname{cr}\left(e_{k}\right) L^{h-j}|X|}$. The dependence on $u$ is in $X$ only; for $G_{j, \text { loc }}$ it is only in an $L^{k-j} r\left(e_{k}\right)$-neighborhood of $x_{1}, x_{2}$. Also, when $x_{1}$ and $x_{2}$ are farther than $L^{k-j} r\left(e_{k}\right)$ from $\Omega^{c}, G_{j, \text { loc }}$ is independent of $\Omega$.

We have developed expansions for $C_{A}^{(j)}(u), W^{(j)}$, and $G_{j}(\Omega, u)$. We now put them together to analyze the expansion of the normalization factors. In the expansion (5.7.4) we put $W^{(j)}=W^{(j, \bar{n})}+\sum_{X} W^{(j)}(X)$. In terms with $l \leqq \bar{n}$ we separate from $\frac{1}{2 l} \operatorname{tr}\left(C_{\Lambda_{10}^{(j)}}^{(j)}\left(u_{k+1}\right) W^{(j, \bar{n})}\right)$ the terms of order $\leqq \bar{n}$ in $e_{j}$. We can write the sum of all these terms as

$$
\sum_{n=1}^{\bar{n}} \frac{1}{n !}\left[\frac{d^{n}}{d e^{\prime n}} \log Z_{\Lambda_{10}^{(j)}}^{(j)}\left(u_{k+1} \exp \left(i e^{\prime} e_{j} \zeta \tilde{A}^{\zeta}\right)\right)\right]_{e^{\prime}=0} .
$$

These will be treated carefully by a resummation. In the other terms we insert the expansion for $C_{\substack{10 \\ 10}}^{(j)}\left(u_{k+1}\right)$; they then take the form $\sum_{X} W^{(j)^{\prime}}(X)$, with

$$
\left|W^{(j)^{\prime}}(X)\right| \leqq e_{j}^{\bar{n}+1-\alpha} e^{-\operatorname{cr}\left(e_{k}\right)|X|^{-}}\left(r\left(e_{k}\right) L^{k-j}\right)^{d}|X| \leqq e_{j}^{\kappa} e^{-\operatorname{cr}\left(e_{k}\right)|X|^{-}} .
$$

We can take $\kappa$ arbitrarily large by increasing $\bar{n}$. The high power of $e_{j}$ beats the big factor $\left(r\left(e_{k}\right) L^{k-j}\right)^{d}$, the volume of an elementary cube measured on the $j$-th scale. This is to account for one free summation on $T_{1}^{(j)}$; all but one such summation is controlled by exponential decay on the $j$-th scale.

We return to the perturbative terms. Resummation in $j$ will be possible only if $\tilde{\tilde{A}}$ is localized to sets like $\bar{\Lambda}_{5}^{(j)} \cap \bar{\Lambda}_{6}^{(j+1) c}$. Thus we write

$$
\tilde{\tilde{A}}=\theta_{k}\left(H_{k \cdot 1 \mathrm{loc}} A^{(k)}+w_{5} A^{\prime}\right)+\sum_{j=-1}^{k-1} \theta_{j}\left(1-\theta_{j+1}\right) w_{5} A^{\prime}=\tilde{\tilde{A}}_{k}+\sum_{j=0}^{k-1} \tilde{\tilde{A}}_{j} .
$$


Here $\theta_{-1}=1$, and each $\tilde{A}_{j}$ is a smooth, small field supported in $\bar{\Lambda}_{5}^{(j)} \cap \bar{\Lambda}_{6}^{(j+1) c}\left(\bar{\Lambda}_{5}^{(k)}\right.$ if $j=k$ ). The low order terms can be written as

$$
\sum_{n=1}^{\bar{n}} \frac{1}{n !}\left[\prod_{\alpha=1}^{n}\left(\sum_{j_{\alpha}=0}^{k} \frac{d}{d e_{j_{\alpha}}^{\prime}}\right) \log Z_{\Lambda_{10}^{(j)}}^{(j)}\left(u_{k+1} \exp \left(i e_{j} \zeta \sum_{l=0}^{k} e_{l}^{\prime} \tilde{\tilde{A}}_{l}^{\zeta}\right)\right)\right]_{e_{l}^{\prime}=0} .
$$

Note that $Z_{\Lambda_{10}^{(j)}}^{(j)}(\ldots)$ depends only on $e_{l}$ for $l \geqq j$. Thus we can write the last expression as

$$
\begin{aligned}
& \sum_{m=j}^{k} \sum_{n=1}^{\bar{n}} \frac{1}{n !} \sum_{\left\{j_{\alpha}\right\}_{\alpha=1}^{n}: \min _{\alpha} j_{\alpha}=m} \\
& \quad \times\left[\prod_{\alpha=1}^{n} \frac{d}{d e_{j_{\alpha}}^{\prime}} \log Z_{\Lambda_{10}^{(j)}}^{(j)}\left(u_{k+1} \exp \left(i e_{j} \zeta \sum_{l=j}^{k} e_{l}^{\prime} \tilde{A}_{l}^{\zeta}\right)\right)\right]_{e_{l}^{\prime}=0} .
\end{aligned}
$$

We write all terms in the form of the expansions derived in this section, except that we write a new expansion analogous to one we gave above for $\left(w_{5} A^{\prime}\right)_{b}^{m}$ :

$$
\prod_{\alpha \in \underline{\alpha}}\left(\theta_{j_{\alpha}}\left(1-\theta_{j_{\alpha}+1}\right) w_{5} A^{\prime}\right)_{b}=\sum_{X} w_{b, \underline{\alpha}}(X) .
$$

Here $\alpha \subset\{1, \ldots, n\}$, and $w_{b, \alpha}(X)$ is also bounded as in (5.7.6).

We insert this expansion in

$$
\left[\prod_{\alpha \in \underline{\alpha}} \frac{d}{d e_{j_{\alpha}}^{\prime}} F_{1, j}\left(\sum_{l=j}^{k} e_{l}^{\prime} \tilde{\tilde{A}}_{l}^{\zeta}\right)\right]_{e_{l}^{\prime}=0} .
$$

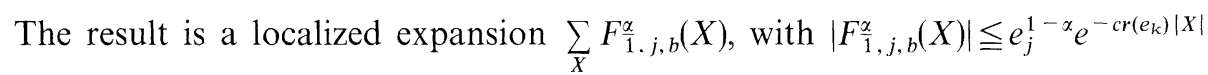
(unless $j_{\alpha}=k$ for all $\alpha \in \underline{\alpha}$, in which case $|X|$ is replaced by $|X|^{-}$). We make the same expansions in $F_{2, j}$. The expansions for $F_{1, j}, F_{2, j}$ are inserted in the low order terms in $V_{j}, G_{j, \text { loc }}\left(u_{k+1} \tilde{\tilde{u}}\right)$, and $\Delta_{k, \text { loc }}\left(u_{k+1} \tilde{\tilde{u}}\right)+a L^{-2} P\left(u_{k+1} \tilde{\tilde{u}}\right)$. Finally, they are inserted into (5.7.10), using (5.7.4) for $\log Z$.

The term $m=j$ is special; we bound that term directly without resummation. The random walk expansion is inserted for $C_{\Lambda^{(j)}}^{(j)}\left(u_{k+1}\right)$, and we obtain an expansion $\sum_{X} W^{(j) \prime \prime}(X)$, with

$$
\left|W^{(j)^{\prime \prime}}(X)\right| \leqq e_{j}^{1-\alpha} e^{-\operatorname{cr}\left(e_{k}\right)|X|}\left|X^{\prime} \cap \Lambda_{5}^{(j)^{\prime}} \cap \Lambda_{6}^{(j+1) c}\right| .
$$

Each term contributing to $W^{(j)^{\prime \prime}}(X)$ must contain at least one kernel $w_{5}$. There is a summation in $\Lambda_{10}^{(j)}$, but since at least one field $\tilde{A}_{j}$ is present, there is an exponential decay on the $j$-th scale localizing summations near $\Lambda_{5}^{(j) \prime} \cap \Lambda_{6}^{(j+1) c}$. This gives rise to the volume factor in the above bound. The volume divergence will be beaten by small factors coming from large fields near $\Lambda_{5}^{(j) \prime} \cap \Lambda_{6}^{(j+1) c}$; we will have available some $e_{j}^{\kappa\left|A_{5}^{(j) \prime} \cap A_{6}^{(j+1) c \mid}\right| / r\left(e_{j}\right)^{d}}$, and since $\kappa\left(\log e_{j}^{-1}\right) r\left(e_{j}\right)^{-d}>\sum_{k=j+1}^{\log _{L}\left(\varepsilon_{0} / \varepsilon\right)} e_{j}^{1-\alpha}$, this is sufficient.

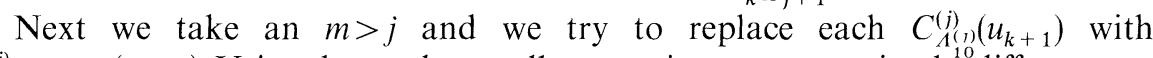
$C_{B_{m-j}\left(\Lambda_{3}^{(m)}\right)}^{(j)}\left(u_{k+1}\right)$. Using the random walk expansions we can write the difference as $\sum_{X} C^{(j)^{\prime}}(X)$, with $\left|C^{(j)^{\prime}}\left(X, x_{1}, x_{2}\right)\right| \leqq e^{-c\left|x_{1}-x_{2}\right|} e^{-\operatorname{cr}\left(e_{k}\right)|X|}$ for $x_{1}, x_{2}$ in $B_{m-j}\left(\Lambda_{4}^{(m)}\right)$. For $m>j$, all operators $C_{\Lambda^{(j)}}^{(j)}\left(u_{k+1}\right)$ in our low order expansion satisfy this restriction. Terms with all $C_{B_{m-J}\left(\Lambda^{(m)}\right)}^{(j)}\left(u_{k+1}\right)$ 's will be considered below. In all other terms, we 
random-walk expand any $C_{B_{m-J}\left(\Lambda^{(m)}\right)}^{(j)}\left(u_{k+1}\right)$ 's, sum over $m$, and obtain

$$
\sum_{X} W^{(j) \prime \prime \prime}(X), \quad \text { with }\left|W^{(j) \prime \prime \prime}(X)\right| \leqq e^{-\operatorname{cr}\left(e_{j}\right)|X|} .
$$

We need to replace the propagators $G_{j, \text { loc }}\left(u_{k+1}\right), G_{j}\left(\square, u_{k+1}\right)$ by $G_{j}\left(\bar{\Lambda}_{2}^{(m)}, u_{k+1}\right)$. They appear in the expansion of $\Delta_{j, 1 \text { oc }}\left(u_{k+1}\right)$ and indirectly in $C^{(j)}\left(u_{k+1}\right)$. We write

$$
\begin{aligned}
G_{j, \text { loc }}\left(u_{k+1}\right)= & G_{j}\left(\bar{\Lambda}_{2}^{(m)}, u_{k+1}\right)+\left(G_{j, \text { loc }}\left(u_{k+1}\right)-G_{j, \text { loc }}\left(\bar{\Lambda}_{2}^{(m)}, u_{k+1}\right)\right) \\
& +\sum_{X} G_{j}\left(\bar{\Lambda}_{2}^{(m)}, X, u_{k+1}\right),
\end{aligned}
$$

and similarly for $G_{j, \text { loc }}\left(\square, u_{k+1}\right)$. We only need to look at this operator in $\bar{\Lambda}_{3}^{(m)}$. There a random walk expansion on the $j$-th scale will yield the usual regularity bounds on the second term on the right with an extra factor $e^{-\operatorname{cr}\left(e_{1}\right)}$. We insert this expansion into $C^{(j)-1}$ to obtain

where

$$
C_{B_{m-j}\left(\Lambda_{3}^{(m)}\right)}^{(j)}\left(u_{k+1}\right)^{-1}=C_{B_{m-J}\left(\Lambda_{3}^{(m)}\right)}^{(j)}\left(\bar{\Lambda}_{2}^{(m)}, u_{k+1}\right)^{-1}+\sum_{X} \Delta_{j}(X)
$$

$$
\begin{aligned}
& C_{B_{m-j}\left(\Lambda_{3}^{(m)}\right)}^{(j)}\left(\bar{\Lambda}_{2}^{(m)}, u_{k+1}\right)^{-1} \\
& \quad=a_{j} I-a_{j}^{2} Q_{j}\left(u_{k+1}\right) G_{j}\left(\bar{\Lambda}_{2}^{(m)}, u_{k+1}\right) Q_{j}^{*}\left(u_{k+1}\right)+a L^{-2} P\left(u_{k+1}\right), \\
& \left|\Delta_{j}\left(X, x_{1}, x_{2}\right)\right| \leqq e^{-c r\left(e_{j}\right)|X|}, \quad=0 \text { if } x_{1} \text { or } x_{2} \notin X .
\end{aligned}
$$

Thus we have

$$
\begin{aligned}
C_{B_{m-\jmath}\left(\Lambda_{3}^{(m)}\right)}^{(j)}\left(u_{k+1}\right)= & C_{B_{m-\jmath}\left(\Lambda_{3}^{(m)}\right)}^{(j)}\left(\bar{\Lambda}_{2}^{(m)}, u_{k+1}\right) \\
& -C_{B_{m-\jmath}\left(\Lambda_{3}^{(m)}\right)}^{(j)}\left(\bar{\Lambda}_{2}^{(m)}, u_{k+1}\right)\left(\sum_{X} \Delta_{j}(X)\right) C_{B_{m-\jmath}\left(\Lambda_{3}^{(m)}\right)}^{(j)}\left(u_{k+1}\right) .
\end{aligned}
$$

This expansion is inserted at each appearance of $C^{(j)}$ in our low order terms. The same analysis is performed when $G_{j}\left(\square, u_{k+1}\right)$ appears instead of $G_{j, \text { loc }}\left(u_{k+1}\right)$. In the leading terms (terms with no $e^{-c r\left(e_{\jmath}\right)}$ from the random walk expansions) we put $\zeta_{j}^{\prime \prime}=1+\left(\zeta_{j}^{\prime \prime}-1\right)$. The leading terms are now

$$
\begin{aligned}
& \sum_{n=1}^{\bar{n}} \frac{1}{n !} \sum_{\left\{j_{\alpha}\right\}: \min _{\alpha} j_{\alpha}=m} \\
& \quad \times\left[\prod_{\alpha=1}^{n} \frac{d}{d e_{j_{\alpha}}^{\prime}} \log Z_{B_{m-1}\left(\Lambda_{3}^{(m)}\right)}^{(j)}\left(\bar{\Lambda}_{2}^{(m)}, u_{k+1} \exp \left(i e_{j} \zeta \sum_{l=j}^{k} e_{l}^{\prime} \tilde{A}_{l}^{\zeta}\right)\right)\right]_{e_{l}^{\prime}=0},
\end{aligned}
$$

where this $Z^{(j)}$ uses the quadratic form in (5.7.11). Remainder terms are again localized - there will be typically some delocalized operators and some localized ones. Thus we random walk expand any $G_{j}\left(\bar{\Lambda}_{2}^{(m)}, u_{k+1}\right)$. Also, we expand any $C_{B_{m-j}\left(\Lambda_{3}^{(m)}\right)}^{(j)}\left(\bar{\Lambda}_{2}^{(m)}, u_{k+1}\right)$ as

$$
\sum_{p=0}^{\infty} C_{B_{m-\jmath}\left(\Lambda_{3}^{(m)}\right)}^{(j)}\left(u_{k+1}\right)\left[\sum_{X} \Delta_{j}(X) C_{B_{m-\jmath}\left(\Lambda_{3}^{(m)}\right)}^{(j)}\left(u_{k+1}\right)\right]^{p},
$$

and finally we random-walk expand all $C_{B_{m-1}\left(A_{3}^{(m)}\right)}^{(j)}\left(u_{k+1}\right)^{\prime}$ s. We gather all terms of this rather complicated expansion of the remainders and sum over $m$, to yield

$$
\sum_{X} W^{(j)(i v)}(X), \quad \text { with }\left|W^{(j)(i v)}(X)\right| \leqq e^{-\operatorname{cr}\left(e_{j}\right)|X|} .
$$


As always, $X$ is a connected union of $L^{k-j} r\left(e_{k}\right)$-cubes, and $W^{(j)(i v)}(X)$ has dependence only on fields in $X, \bar{X}$, or $B_{k-j}(X)$.

We make a final change in the leading terms, namely we replace

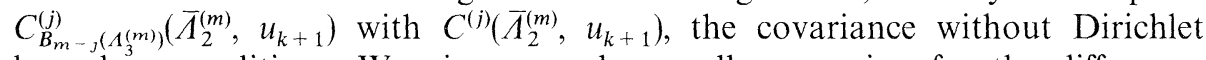
boundary conditions. We give a random walk expansion for the difference, $\sum_{X} C^{(j) \prime \prime}(X)$. It is actually a double expansion, since each term in the usual random walk expansion still depends on $\bar{\Lambda}_{2}^{(m)}$ through the basic quadratic form [which involves $\left.G_{j}\left(\bar{\Lambda}_{2}^{(m)}, u_{k+1}\right)\right]$ and through operators $C_{\square}^{(j)}\left(\bar{\Lambda}_{2}^{(m)}, u_{k+1}\right)$. However, each of these can be expanded as described earlier, yielding terms $C^{(j) \prime}(X)$ with proper locality properties, and obeying the following bounds:

$$
\left|C^{(j) \prime \prime}\left(X, x_{1}, x_{2}\right)\right| \leqq e^{--c r\left|x_{1}-x_{2}\right|} e^{-\operatorname{cr}\left(e_{J}\right)|X|^{-}} e^{-\operatorname{cdist}\left(\left\{x_{1}, x_{2}\right\}, A_{3}^{(m) c}\right)} .
$$

Leading terms are now given as in (5.7.12) but with no Dirichlet boundary conditions. Finally, remainder terms are expanded out completely. All remainder terms have at least one operator $C^{(j) \prime \prime}(X)$, which provides exponential localization to $B_{m-j}\left(\Lambda_{3}^{(m)}\right)^{c}$. The field $\widetilde{A}_{m}$ is supported in $\bar{\Lambda}_{5}^{(m)}$, thus all terms have at least a factor $e^{-\operatorname{cr}\left(e_{j}\right)}$. Thus we can sum all the remainder terms into

$$
\sum_{X} W^{(j)(v)}(X), \quad \text { with }\left|W^{(j)(v)}(X)\right| \leqq e^{-\operatorname{cr}\left(e_{j}\right)|X|} .
$$

Now the leading terms can be rescaled to the $L^{j-m}$-lattice, and we sum over $j<m$. All the changes we have made allow us now to compose the normalization factors as

$$
\prod_{j=0}^{m-1} Z^{(j), L^{j-m}}\left(\bar{\Lambda}_{2}^{(m)}, u\right)=Z_{m}\left(\bar{\Lambda}_{2}^{(m)}, u\right) \cdot \text { const },
$$

where the $m$-step Gaussian normalization factor $Z_{m}$ arises as in Eq. (2.40) of [7]. We obtain the perturbative expansion

$$
\begin{aligned}
& \sum_{n=1}^{\bar{n}} \sum_{\left\{j_{\alpha}\right\}: \min _{\alpha} j_{\alpha}=m} \\
& \quad \times\left[\prod_{\alpha=1}^{n} \frac{d}{d e_{j_{\alpha}}^{\prime}} \log Z_{m}\left(\bar{\Lambda}_{2}^{(m)}, u_{k+1} \exp \left(i e_{m} L^{-m} \sum_{l=m}^{k} e_{l}^{\prime} \tilde{\tilde{A}}_{l}^{L^{-m}}\right)\right)\right]_{e^{\prime}=0} .
\end{aligned}
$$

The diagrams in this expansion are covered by our theorems on the perturbation expansion. The point is that various Ward identities and symmetries necessary to obtain good bounds can only be seen in this resummed form of perturbation theory. We give the random walk expansion for the propagator $G_{m}\left(\bar{\Lambda}_{2}^{(m)}, u_{k+1}\right)$. There is at least one factor $e_{m}$ in all terms, and a free summation in $\Lambda_{5}^{(m)}$ $\cap \Lambda_{6}^{(m+1) c}(m<k)$ or $\Lambda_{5}^{(m)}(m=k)$. Thus we can write the perturbation expansion for $m<k$ as $\sum_{X} W^{(m)(v i)}(X)$, with

$$
\left|W^{(m)(v i)}(X)\right| \leqq e_{m}^{1-\alpha} e^{-c r\left(e_{k}\right)|X|}\left|X \cap \Lambda_{5}^{(m) \prime} \cap \Lambda_{6}^{(m+1) c}\right| .
$$

As for the $W^{(j)^{\prime \prime}}$ terms, the volume factor will be beaten by convergence factors from the large field region $\Lambda_{5}^{(m) \prime} \cap \Lambda_{6}^{(m+1) c}$. 
The term $m=k$ is treated slightly differently. We decompose $\tilde{\tilde{A}}_{k}^{\eta}$ into $\theta_{k} H_{k, \text { loc }} A^{(k)}+\theta_{k} w_{5} A^{\prime}$. Terms with one or more $w_{5} A^{\prime}$ field are expanded as in the $m<k$ case. In terms with all $\theta_{k} H_{k, \text { loc }} A^{(k)}$ fields, we expand the propagators as before, leaving in the main terms the localized propagator $G_{k \text {, loc }}\left(u_{k+1}\right)$. The result is our standard perturbative expansion in the field $\theta_{k} H_{k, \text { loc }} A^{A(k)}$, which we denote $Q^{(k)}\left(u_{k+1}, \quad \theta_{k} H_{k \text {.loc }} A^{(k)}\right)$. The remainder terms become $\sum_{X} W^{(k)(v i)}(X)$, with $\left|W^{(k)(v i)}(X)\right| \leqq e^{-\operatorname{cr}\left(e_{k}\right)|X|}$.

We can summarize the results of this section as follows:

$$
\begin{aligned}
& \prod_{j=0}^{k-1} Z_{\Lambda_{10}(j)}^{(j)}\left(u_{k+1} \tilde{\tilde{u}}\right) \\
& \quad=\prod_{j=0}^{k-1} Z_{\Lambda_{10}^{(j)}}^{(j)}\left(u_{k+1}\right) \exp \left[-Q^{(k)}\left(u_{k+1}, \theta_{k} H_{k, 1 \mathrm{loc}} A^{(k)}-\sum_{X} W_{2}^{(k)}(X)\right],\right.
\end{aligned}
$$

where $X$ is a connected union of $r\left(e_{k}\right)$-cubes in $T_{1}^{(k)}$,

$$
\begin{gathered}
W_{2}^{(k)}(X)=\sum_{j=0}^{k} W^{(j)^{\prime}}(X)+\ldots+W^{(j)(v i)}(X), \\
\left|W_{2}^{(k)}(X)\right| \leqq e_{k}^{\kappa} e^{-c r\left(e_{k}\right)|X|^{-}}+\sum_{j<k} e_{j}^{1-\alpha} e^{-c r\left(e_{k}\right)|X|}\left|B_{k-j-1}(X) \cap \Lambda_{5}^{(j) \prime} \cap \Lambda_{6}^{(j+1) c}\right|,
\end{gathered}
$$

and

$$
\begin{aligned}
& Q^{(k)}\left(u_{k+1}, \theta_{k} H_{k, \operatorname{loc}} A^{(k)}\right) \\
& \quad=\sum_{n=1}^{n} \frac{d^{n}}{d e^{\prime n}} \log \left[\left.\int d \phi\right|_{\bar{A}_{4}^{(k)}} \exp \left(-\frac{1}{2}\left\langle\phi, G_{k, \operatorname{loc}}\left(u_{k+1} e^{i e^{\prime} e_{k} \theta_{k} \theta_{k} H_{k, 10 c} A^{(k)}}\right)^{-1} \phi\right\rangle\right)\right]_{e^{\prime}=0 .} .
\end{aligned}
$$

\subsection{Scalar Field Translation}

The scalar field quadratic forms, after all our manipulations with the gauge field, are as follows:

$$
\frac{1}{2}\left\langle\Lambda_{8}^{(k-1)^{\prime}} \phi, A_{k, \text { loc }}\left(\tilde{u}_{k+1}\right) \Lambda_{8}^{(k-1)^{\prime}} \phi\right\rangle+\frac{1}{2} a L^{-2}\left\langle\psi-Q\left(\tilde{u}_{k+1}\right) \phi, \psi-Q\left(\tilde{u}_{k+1}\right) \phi\right\rangle .
$$

To eliminate most of the linear term $\left\langle\psi, Q\left(\tilde{u}_{k+1}\right) \phi\right\rangle$ in the small field region, we make a translation

$$
\phi=\phi^{(k)}+a L^{-2} \Lambda_{7}^{(k)} C_{\mathrm{loc}}^{(k)}\left(u_{k+1}\right) Q^{*}\left(u_{k+1}\right) \psi .
$$

(Recall that $u_{k+1}=\tilde{u}_{k+1}$ in $\bar{\Lambda}_{6}^{(k)}$.)

The terms quadratic in $\phi^{(k)}$ are then

$$
\frac{1}{2}\left\langle\Lambda_{8}^{(k-1)^{\prime}} \phi^{(k)},\left(\Lambda_{k \cdot \text { loc }}\left(\tilde{u}_{k+1}\right)+a L^{-2} P\left(\tilde{u}_{k+1}\right)\right) \Lambda_{8}^{(k-1)^{\prime}} \phi^{(k)}\right\rangle+\mathscr{Q}_{4},
$$

where

$$
\mathscr{Q}_{4}=\frac{1}{2} a L^{-2}\left\langle\phi^{(k)}, \Lambda_{8}^{(k-1) ` c} P\left(\tilde{u}_{k+1}\right) \phi^{(k)}\right\rangle .
$$

In the cross terms between $\phi^{(k)}$ and $\psi$, we write $\psi=\Lambda_{8}^{(k) \prime} \psi+\Lambda_{8}^{(h) c} \psi$. The terms with $\Lambda_{8}^{(k) \prime c} \psi$ define $\mathscr{2}_{5}$, a form localized near $\Lambda_{8}^{(k) \prime c}$. The other terms can be written as $\left\langle\phi^{(k)}, w_{6} \psi\right\rangle$, with $w_{6}$ a small kernel with range less than $r\left(e_{k}\right)$. This is because (5.8.1) would eliminate entirely the linear term were it not for the localizations. 
In the terms quadratic in $\psi$, we again combine all terms involving $\Lambda_{8}^{(k) \prime c} \psi$ into a form $\mathscr{Q}_{6}$ localized near $\Lambda_{8}^{(k) c}$. The remaining terms become

$$
\frac{1}{2} a L^{-2}\left\langle\Lambda_{8}^{(k) \prime} \psi,\left(I-a L^{-2} Q\left(u_{k+1}\right) C_{\mathrm{loc}}^{(k)}\left(u_{k+1}\right) Q\left(u_{k+1}\right)^{*}\right) \Lambda_{8}^{(k) \prime} \psi\right\rangle+\frac{1}{2}\left\langle\psi, w_{7}^{\prime} \psi\right\rangle,
$$

with $w_{7}^{\prime}$ another small, local kernel. We apply the identity [7]

$$
\begin{aligned}
& a L^{-2} I-a^{2} L^{-4} Q\left(u_{k+1}\right) C^{(k)}\left(u_{k+1}\right) Q\left(u_{k+1}\right)^{*} \\
& \quad=a_{k} L^{-2} I-a_{k}^{2} L^{-4} Q_{k+1}\left(u_{k+1}\right) G_{k+1}^{n}\left(u_{k+1}\right) Q_{k+1}\left(u_{k+1}\right)^{*},
\end{aligned}
$$

but in a localized version with $C_{\text {loc }}^{(k)}$ and $G_{k+1, \text { loc }}^{\eta}$ and with another small kernel $w_{7}^{\prime \prime}$ on the right. This yields the desired form $\Delta_{k+1, \text { loc }}^{L}\left(u_{k+1}\right)$, and so we obtain

$$
\frac{1}{2}\left\langle\Lambda_{8}^{(k) \prime} \psi, \Delta_{k+1, \operatorname{loc}}^{L}\left(u_{k+1}\right) \Lambda_{8}^{(k) \prime} \psi\right\rangle+\frac{1}{2}\left\langle\psi, w_{7} \psi\right\rangle \text {, with } \quad w_{7}=w_{7}^{\prime}+w_{7}^{\prime \prime} .
$$

To summarize, we have written

$$
\begin{gathered}
\frac{1}{2}\left\langle\Lambda_{8}^{(k-1)^{\prime}} \phi, \Delta_{k, \text { loc }}\left(\tilde{u}_{k+1}\right) \Lambda_{8}^{(k-1)^{\prime}} \phi\right\rangle+\frac{1}{2} a L^{-2}\left\langle\psi-Q\left(\tilde{u}_{k+1}\right) \phi, \psi-Q\left(\tilde{u}_{k+1}\right) \phi\right\rangle \\
=\mathscr{P}_{4}+\mathscr{Q}_{5}+\mathscr{2}_{6}+\frac{1}{2}\left\langle\Lambda_{8}^{(k-1)^{\prime}} \phi^{(k)},\left(\Delta_{k, \text { loc }}\left(\tilde{u}_{k+1}\right)+a_{1}^{I^{-2}} P\left(\tilde{u}_{k+1}\right)\right) \Lambda_{8}^{(k-1)^{\prime}} \phi^{(k)}\right\rangle \\
+\frac{1}{2}\left\langle\Lambda_{8}^{(k) \prime \prime} \psi, \Delta_{k+1, \text { loc }}^{L}\left(u_{k+1}\right) \Lambda_{8}^{(k) \prime} \psi\right\rangle+\left\langle\phi^{(k)}, w_{6} \psi\right\rangle+\frac{1}{2}\left\langle\psi, w_{7} \psi\right\rangle,
\end{gathered}
$$

with $w_{6}, w_{7}$ small local kernels, and with $\mathscr{Q}_{4}, \mathscr{Z}_{5}, \mathscr{Q}_{6}$ localized near $\Lambda_{8}^{(k) c c}$.

\subsection{Bounds on Fluctuation and Block Fields}

As we remarked earlier, the restrictions on $u(p)$ and the gauge field renormalization transformations imply that

$$
\left|\frac{1}{i e_{k}} \log v(p)\right|=|f(p)| \leqq c p\left(e_{k}\right), \quad p \in \Lambda_{0}^{(k) * * *} .
$$

Also, bounds on $\phi$ and $\psi-Q\left(u_{k}\right) \phi$ imply that for $y \in \Lambda_{0}^{(k) \prime}$,

$$
\begin{gathered}
|\psi(y)| \leqq c p\left(e_{k}\right) \lambda_{k}^{-1 / 4}, \quad\left(L^{k} \varepsilon\right)^{d}<\lambda, \\
|| \psi(y)\left|-(8 \lambda)^{-1 / 2}\left(L^{k} \varepsilon\right)^{(d-2) / 2}\right| \leqq c p\left(e_{k}\right)\left(L^{k} \varepsilon\right)^{-1}, \quad\left(L^{k} \varepsilon\right)^{d} \geqq \lambda .
\end{gathered}
$$

Next, we wish to prove that

$\left|u_{k+1}\left(\left\langle b_{-}, b_{+}\right\rangle\right) \psi\left(b_{+}\right)-\psi\left(b_{-}\right)\right| \equiv\left|\left(D_{\bar{u}_{k+1}} \psi\right)(b)\right| \leqq c p\left(e_{k}\right), \quad b \in \Lambda_{0}^{(k) \prime *}$.

We prove the bound first for $D_{\bar{u}_{k}} \psi$ (before the gauge transformation of Sect. 5). Our bounds on $\psi-Q\left(u_{k}\right) \phi$ reduce this to estimating

$$
\left|u_{k}\left(\Gamma_{b_{-}, x}\right) \phi(x)-u_{k}\left(\left\langle b_{-}, b_{+}\right\rangle\right) u_{k}\left(\Gamma_{b_{+}, x^{\prime}}\right) \phi\left(x^{\prime}\right)\right|
$$

for any $x \in B\left(b_{-}\right), x^{\prime} \in B\left(b_{+}\right)$. This is proven with several applications of our bounds on $D_{\bar{u}_{k}} \phi$. In going from $u_{k}$ to $u_{k+1}$ we made a gauge transformation and removed some small fields. Also, the gauge transformation was not quite compensated by a rotation of $\psi$. Thus in going from the old $\left|D_{\bar{u}_{k}} \psi\right|$ to the new $\left|D_{\bar{u}_{k+1}} \psi\right|$ we make errors of the order of $c e_{k} p\left(e_{k}\right)^{3} \lambda_{k}^{-1 / 4},\left(L^{k} \varepsilon\right)^{d}<\lambda$ or

$$
c e_{k} p\left(e_{k}\right)^{3}\left(\lambda^{-1 / 2}\left(L^{k} \varepsilon\right)^{(d-2) / 2}+\left(L^{k} \varepsilon\right)^{-1}\right), \quad\left(L^{k} \varepsilon\right)^{d} \geqq \lambda .
$$


In both cases this is bounded by $e^{\beta}\left(L^{k} \varepsilon / \varepsilon_{0}\right)^{1 / 4-\alpha}$ - see the discussion below of the bounds on interaction terms. The desired bound follows.

These bounds allow us to insert the following characteristic functions:

$$
\begin{aligned}
\chi_{k+1, \Lambda_{0}^{(k)}}= & \prod_{p \in \Lambda_{0}^{(k) * * *}} \chi\left(c e_{k} p\left(e_{k}\right),|v(p)-1|\right) \\
& \times \prod_{y \in \Lambda_{0}^{(k)},} \chi\left(c p\left(e_{k}\right) \lambda_{k}^{-1 / 4},|\psi(y)|\right) \prod_{b \in \Lambda_{0}^{(k) \prime *}} \chi\left(c p\left(e_{k}\right),\left|\left(D_{\bar{u}_{k+1}} \psi\right)(b)\right|\right)
\end{aligned}
$$

and the integral is unchanged. If $\left(L^{k} \varepsilon\right)^{d} \geqq \lambda$, the bound on $\psi$ is replaced with $\chi\left(c p\left(e_{k}\right)\left(L^{k} \varepsilon\right)^{-1},|| \psi(y)\left|-(8 \lambda)^{-1 / 2}\left(L^{k} \varepsilon\right)^{(d-2) / 2}\right|\right)$.

We remarked earlier that $A^{\prime}$ is small in $\Lambda_{1}^{(k) *}$. We then defined $A^{(k)}=A^{\prime}$ $+\Lambda_{4}^{(k) *} L^{-2} C_{\mathrm{loc}}^{(k)} H_{k .10 \mathrm{l}}^{*} \partial^{*} Q_{k+1}^{e *} f$. Since $f$ is small and $H_{k, \text { loc }}$ is regular, we have that

$$
\left|A_{b}^{(k)}\right| \leqq c p\left(e_{k}\right), \quad b \in \Lambda_{1}^{(k) *} .
$$

We want a similar bound for $\phi^{(k)}(x), x \in \Lambda_{7}^{(k)}$. Note that $C_{\text {loc }}^{(k)}\left(u_{k+1}\right)$ is almost equal to $C^{(k)}\left(u_{k+1}\right)$. Thus we have that in $\Lambda_{7}^{(k)}$, say

$$
a L^{-2} C_{\text {loc }}^{(k)}\left(u_{k+1}\right) Q\left(u_{k+1}\right)^{*} \psi=Q\left(u_{k+1}\right)^{*} \psi+O\left(p\left(e_{k}\right)\right),
$$

(the corresponding statement with $C^{(k)}\left(u_{k+1}\right)$ was proven in [8, Eq. (2.113)]. Using arguments like the ones we used to bound $D_{\bar{u}_{k+1}} \psi$, we can replace $Q\left(u_{k+1}\right)^{*} \psi$ with $\phi$ in this bound. This proves that

$$
\left|\varphi^{(k)}(x)\right| \leqq c p\left(e_{k}\right), \quad x \in \Lambda_{7}^{(k) *} .
$$

The bounds (5.9.4), (5.9.5) allow us to insert the characteristic functions

$$
\chi_{\Lambda_{7}^{(k)}}^{\prime}=\prod_{b \in \Lambda_{7}^{(k) *}} \chi\left(c p\left(e_{k}\right), A^{(k)}\right) \prod_{x \in \Lambda_{7}^{(k)}} \chi\left(c p\left(e_{k}\right), \phi^{(k)}\right)
$$

without changing anything.

We note that the restrictions implied by $\chi_{\Lambda_{0}^{(k)}}$ are stronger than the corresponding restrictions in $\chi_{k, \Lambda_{0}^{(k-1)}}$, in $\Lambda_{0}^{(k)}$. [When $\left(L^{k} \varepsilon\right)^{d} \geqq \lambda$, we use the inequality $(8 \lambda)^{-1 / 2}\left(L^{k} \varepsilon\right)^{(d-2) / 2}+p\left(e_{k}\right)\left(L^{k} \varepsilon\right)^{-1} \leqq c p\left(e_{k}\right) \lambda_{k}^{-1 / 4}$.] Thus we can replace $\chi_{k, A_{0}^{(k-1)^{\prime}}}$

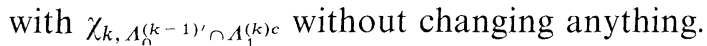

Let us summarize the operations performed so far by using the concluding formulae in the last several sections to write a complete expression for our density.

$$
\begin{aligned}
& \varrho_{k+1}^{L}(v, \psi) \\
& =\left.\sum_{\left\{X_{\omega}\right\}} \sum_{\Lambda_{0}^{(k)}} \int d u^{(k)} d \phi^{(k)} \delta_{\mathrm{Ax}}\left(u^{(k)}\right) \delta_{\Lambda_{1}^{(k) * *}}\left(v / Q u^{(k)}\right) \delta_{\Lambda_{1}^{(k) * *}}\left(\frac{e_{k}}{2 \pi} Q A^{(k)}\right) \int \prod_{j=0}^{k-1} d u^{(j)}\right|_{\Lambda_{10}^{(j) c *}} \\
& \times \zeta_{\Lambda_{0}^{(k) c}} \chi_{\Lambda_{0}^{(k)}} \chi_{k \cdot \Lambda_{0}^{(k-1)^{\prime}} \cap A_{1}^{(k) c}} \chi_{\left.k+1, \Lambda_{0}^{(k)}\right)^{\prime}} \chi_{7}^{(k)} \prod_{\omega} g_{k}\left(X_{\omega}\right) \prod_{\sigma}\left(F_{k, \text { loc }}^{(\bar{m})}\left(X_{\sigma}\right)+\widetilde{F}_{\operatorname{loc}}^{(k)}\left(X_{\sigma}\right)\right) \\
& \times \prod_{j=0}^{k-1}\left[Z_{\Lambda_{10}^{(j) c * c}}^{(j)} Z_{\Lambda_{10}^{(j)}}^{(j)}\left(u_{k+1}\right)\right] \exp \left[-\frac{1}{2}\left\langle\Lambda_{1}^{(k) * * \partial} \partial A^{(k)}, \sigma_{k, \text { loc }} \Lambda_{1}^{(k) * *} \partial A^{(k)}\right\rangle\right.
\end{aligned}
$$

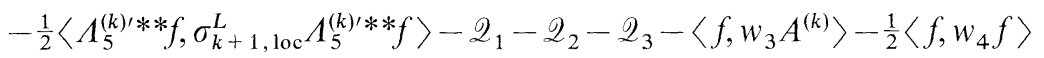

$$
\begin{aligned}
& -\mathscr{2}_{4}-\mathscr{2}_{5}-\mathscr{2}_{6}-\frac{1}{2}\left\langle\Lambda_{8}^{(k-1)^{\prime}} \phi^{(k)},\left(\Delta_{k, \text { loc }}\left(\tilde{u}_{k+1}\right)+a L^{-2} P\left(\tilde{u}_{k+1}\right)\right) \Lambda_{8}^{(k-1)^{\prime}} \phi^{(k)}\right\rangle \\
& -\frac{1}{2}\left\langle\Lambda_{8}^{(k) \prime} \psi, \Lambda_{k+1, \operatorname{loc}}^{L}\left(u_{k+1}\right) \Lambda_{8}^{(k) \prime} \psi\right\rangle-\left\langle\phi^{(k)}, w_{6} \psi\right\rangle \\
& -\frac{1}{2}\left\langle\psi, w_{7} \psi\right\rangle-\mathscr{E}_{k}-E^{(k)}-\mathscr{P}_{k, \operatorname{loc}}\left(\Lambda_{8}^{(k-1)}, \tilde{u}_{k+1}\right)-R^{(k)}\left(u_{k+1}, \theta_{k} H_{k, \mathrm{loc}} A^{(k)}\right) \\
& \left.-\sum_{\square} W_{1}^{(k)}(\square)-Q^{(k)}\left(u_{k+1}, \theta_{k} H_{k, \text { loc }} A^{(k)}\right)-\sum_{X} W_{2}^{(k)}(X)\right] \text {. }
\end{aligned}
$$




\subsection{The Interaction for the Fluctuation Fields}

Having made the scalar field translation, we regard the terms $\mathscr{P}_{k, \text { loc }}, R^{(k)}, Q^{(k)}, F_{k, \text { loc }}^{(\bar{m})}$ as polynomials in $\phi^{(k)}, A^{(k)}$. We make some small changes and localizations in order to obtain the standard form of the fluctuation field interaction in $\Lambda_{8}^{(k)}$.

We have the external field

$$
a_{k} a L^{-2} G_{k, \text { loc }}\left(\tilde{u}_{k+1}\right) Q_{k}^{*}\left(\tilde{u}_{k+1}\right) \Lambda_{7}^{(k)} C_{\text {loc }}^{(k)}\left(u_{k+1}\right) Q^{*}\left(u_{k+1}\right) \psi
$$

appearing in the diagrams in $\mathscr{P}_{k, \text { loc }}, R^{(k)}, F_{k, \text { loc }}^{(\bar{m})}$. The first we leave alone, whereas in the second we localize the field to $\bar{\Lambda}_{8}^{(k)}$ and replace it with

$$
a_{k+1} L^{-2} G_{k+1,10 c}^{\eta}\left(u_{k+1}\right) Q_{k+1}^{*}\left(u_{k+1}\right) \psi+w_{8} \psi .
$$

The kernel $w_{8}$ is local and small with small derivatives and Hölder derivatives. This is accomplished in the usual fashion by replacing $G_{k, \text { loc }}\left(u_{k+1}\right), C_{\text {loc }}^{(k)}\left(u_{k+1}\right)$ with the corresponding operators with Neumann boundary conditions on an $r\left(e_{k}\right)$-cube $\square$. The propagator composition formula $[7$, Eq. $(2.41)]$ is applied, and $G_{k+1}^{n}\left(\square, u_{k+1}\right)$ is localized again.

We localize all vertices to $\bar{\Lambda}_{8}^{(k)}$; vector field legs at a vertex are multiplied by a smooth function $\widetilde{\theta}_{k}$ changing from 0 to 1 in a neighborhood of $\bar{\Lambda}_{8}^{(k) c}$. We also remove all diagrams whose combined order in $\lambda^{1 / 2}$ and $e$ is greater than $\bar{n}$. We still consider all $P_{k}$ vertices together; any $P_{k}^{(l)}$ vertex is considered as one power of $\lambda$. Each mass renormalization counterterm is written graphically and powers counted accordingly. The result is the interaction $V^{(k)}\left(\Lambda_{8}^{(k)}, u_{k+1}, A^{(k)}, \phi^{(k)}\right)$, and

$$
\begin{aligned}
& \mathscr{P}_{k .1 \mathrm{loc}}\left(\Lambda_{8}^{(k-1)}, \tilde{u}_{k+1}\right)+R^{(k)}\left(u_{k+1}, \theta_{k} H_{k \cdot 1 \mathrm{loc}} A^{(k)}\right)+Q^{(k)}\left(u_{k+1}, \theta_{k} H_{k, \mathrm{loc}} A^{(k)}\right) \\
& =\mathscr{P}_{k \cdot 1 \mathrm{loc}}\left(\Lambda_{8}^{(k-1)}, \Lambda_{8}^{(k) c}, \tilde{u}_{k+1}\right)+R^{(k)}\left(\Lambda_{8}^{(k) c}, u_{k+1}, \theta_{k} H_{k, 1 \mathrm{loc}} A^{(k)}\right. \\
& \quad+Q^{(k)}\left(\Lambda_{8}^{(k) c}, u_{k+1}, \theta_{k} H_{k, \operatorname{loc}} A^{(k)}\right)+V^{(k)}\left(\Lambda_{8}^{(k)}, u_{k+1}, A^{(k)}, \phi^{(k)}\right)+\sum_{\square} W_{3}^{(k)}(\square) .
\end{aligned}
$$

Here in writing $\Lambda_{8}^{(k) c}$ we mean that only the terms without proper localizations are included. The terms $W_{3}^{(k)}(\square)$ contain terms localized near the $r\left(e_{k}\right)$-cube $\square$ which involve the small kernel $w_{8}$ or have high powers of coupling constants. We have an estimate

$$
\left|W_{3}^{(k)}(\square)\right| \leqq\left[e^{\beta}\left(L^{k} \varepsilon / \varepsilon_{0}\right)^{1 / 4-\alpha}\right]^{\bar{n}+1} \leqq e^{\bar{n} \beta}\left(L^{k} \varepsilon / \varepsilon_{0}\right)^{\kappa},
$$

with $\kappa>d$ as large as desired if $\bar{n}>\bar{n}(\kappa)$. This estimate comes from our analysis of the perturbation expansion and the restrictions on the fields. We find that each vertex results in at least a factor $e^{\beta}\left(L^{k} \varepsilon / \varepsilon_{0}\right)^{1 / 4-\alpha}$.

Estimates on $V^{(k)}, Q^{(k)}, R^{(k)}$ follow from the same analysis. When localized for example to a cube of size $r\left(e_{k}\right)$, all terms [except for $P_{k}\left(a_{k+1} L^{-2} G_{k+1, \operatorname{loc}}^{\eta}\left(u_{k+1}\right) Q_{k+1}^{*}\left(u_{k+1}\right) \psi\right]$ are bounded by $e^{\beta}\left(L^{k} \varepsilon / \varepsilon_{0}\right)^{1 / 4-\alpha}$, with $\alpha, \beta$ small and positive.

In a similar fashion we modify the external scalar fields in $F_{k, \text { loc }}^{(\bar{m})}$ and eliminate diagrams of order higher than $\bar{m}$. Thus we write

$$
F_{k .1 \mathrm{loc}}^{(\bar{m})}\left(X_{\sigma}\right)=F_{k .1 \mathrm{loc}}^{\bar{m}}\left(X_{\sigma}\right)+\tilde{\tilde{F}}_{k, \text { loc }}\left(X_{\sigma}\right),
$$

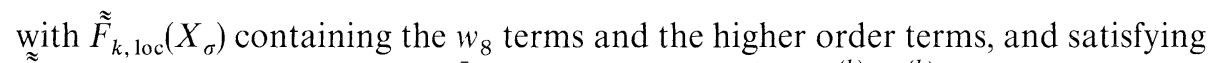
$\tilde{\widetilde{F}}_{k, \text { loc }}\left(X_{\sigma}\right) \leqq c(F)$. We regard $F_{k, \text { loc }}^{\bar{m}}$ as a polynomial in $A^{(k)}, \phi^{(k)}$. 


\subsection{Mayer Expansion I}

In this section we expand irrelevant terms down from the exponent. This operation is done to simplify the structure of the integral in the region free of irrelevant terms.

Let us combine the irrelevant terms as follows:

$$
\begin{aligned}
\left\langle f, w_{3} A^{(k)}\right\rangle & +\frac{1}{2}\left\langle f, w_{4} f\right\rangle+\left\langle\phi^{(k)}, w_{6} \psi\right\rangle+\frac{1}{2}\left\langle\psi, w_{7} \psi\right\rangle+\sum_{\square} W_{1}^{(k)}(\square)+\sum_{X} W_{2}^{(k)}(X) \\
& +\sum_{\square} W_{3}^{(k)}(\square)=\sum_{X} W_{4}^{(k)}(X),
\end{aligned}
$$

with $W_{4}^{(k)}(X)$ containing terms with dependence in $X$. We combine the estimates on the above terms to obtain

$$
\begin{aligned}
\left|W_{4}^{(k)}(X)\right| \leqq & {\left[e^{\beta}\left(L^{k} \varepsilon / \varepsilon_{0}\right)^{1 / 4-\alpha}\right]^{\bar{n}+1} e^{-c r\left(e_{k}\right)|X|} } \\
& +\sum_{j<k} e_{j}^{1-\alpha} e^{-c r\left(e_{k}\right)|X|}\left|B_{k-j-1}(X) \cap \Lambda_{5}^{(j) \prime} \cap \Lambda_{6}^{(j+1) c}\right|
\end{aligned}
$$

We can only Mayer-expand small terms, therefore we parcel up $W_{4}^{(k)}(X)$ into manageable chunks. It is a simple matter to decompose $W_{4}^{(k)}(X)$ as follows

$$
W_{4}^{(k)}(X)=\sum_{j<k} \sum_{x_{j} \in B_{k-j-1}(X) \cap A_{5}^{(j)} \cap A_{6}^{(j+1) c}} W_{4, j}^{(k)}\left(x_{j}, X\right)+W_{4, k}^{(k)}\left(x_{k}, X\right) .
$$

Here $x_{k}$ is some distinguished point in $X$ (for unity of notation) and

$$
\begin{gathered}
\left|W_{4 . j}^{(k)}\left(x_{j}, X\right)\right| \leqq e_{j}^{1-\alpha} e^{-c r\left(e_{k}\right)|X|} \\
\left|W_{4, k}^{(k)}\left(x_{k}, X\right)\right| \leqq\left[e^{\beta}\left(L^{k} \varepsilon / \varepsilon_{0}\right)\right]^{\bar{n}+1} e^{-c r\left(e_{k}\right)|X|^{-}} .
\end{gathered}
$$

The Mayer expansion is the usual identity

$$
\exp \left(-\sum_{X} W_{4}^{(k)}(X)\right)=\sum_{S_{4}} \prod_{(j, x, X) \in S_{4}}\left(e^{-W_{4}^{(k)}\left(x_{j}, X\right)}-1\right)
$$

Let $\widetilde{S}_{4}$ be the set of all triplets $\left(j, x_{j}, X\right)$ that arise in the above decomposition of $W_{4}^{(k)}(X)$, for any $X$. Then $S_{4}$ is summed over subsets of $\widetilde{S}_{4}$. Note that $e^{-W_{4, j}^{(k)}\left(x_{J}, X\right)}-1$ satisfies the same bound as $W_{4, j}^{(k)}\left(x_{j}, X\right)$.

To see what kind of control we have over this expansion, let us do a typical estimate of the type we need:

$$
\begin{aligned}
& \left|\sum_{S_{4}=\left\{\left(j_{\alpha}, x_{j}, \alpha, X_{\alpha}\right)\right\}: \cup X_{\alpha} X_{\alpha}=X \alpha} \prod_{\alpha}\left(e^{-W_{4, j}^{(k)}\left(x_{1, \alpha}, X_{\alpha}\right)}-1\right)\right| \\
& \leqq \exp \left(\sum_{j<k} e_{j}^{1-\alpha} e^{-\operatorname{cr}\left(e_{k}\right)}\left|B_{k-j-1}(X) \cap \Lambda_{5}^{(j) \prime} \cap \Lambda_{6}^{(j+1) c}\right|\right)\left[e^{\beta}\left(L^{k} \varepsilon / \varepsilon_{0}\right)\right]^{(\bar{n}+1)|X|} .
\end{aligned}
$$

We consider first sums over $X_{\alpha}$ such that $x_{j, \alpha}=x_{j}$. A combinatoric factor $c^{\left|X_{\alpha}\right|}$ controls each sum over $X_{\alpha}$, and can be absorbed into the factors $e^{-\operatorname{cr}\left(e_{k}\right)|X|^{-}}$in our bounds on $e^{-W^{(k)}\left(x_{j}, X\right)}-1$. If there are $n$ such sets, we use $n$ factors of $e_{j}^{1-\alpha} E^{-c r\left(e_{k}\right)}$, $j<k$. The resulting estimate has a factor

$$
\sum_{n=0}^{\infty}\left(e_{j}^{1-\alpha} e^{-c r\left(e_{k}\right)}\right)^{n} \leqq \exp \left(e_{l}^{1-\alpha} e^{-c^{\prime} r\left(e_{k}\right)}\right)
$$

at each $x_{j}, j<k$, or $O(1), j=k$. There remains a factor $\left[e^{\beta}\left(L^{k} \varepsilon / \varepsilon_{0}\right)\right]^{(\bar{n}+1)|X|}$ from a worst-case analysis of the unused small factors, and (5.11.3) follows. 
We also expand out the observable:

$$
\prod_{\sigma}\left(F_{k, 1 \mathrm{loc}}^{\bar{m}}\left(X_{\sigma}\right)+\widetilde{F}_{k, \text { loc }}\left(X_{\sigma}\right)+\tilde{\tilde{F}}_{k, \text { loc }}\left(X_{\sigma}\right)\right)=\sum_{\tilde{\sigma}_{1}} \prod_{\sigma_{1} \in \tilde{\sigma}_{1}} F_{k .1 \mathrm{loc}}^{\bar{m}}\left(X_{\sigma_{1}}\right) \prod_{\sigma \notin \tilde{\sigma}_{1}} F_{k, \text { loc }}^{\prime}\left(X_{\sigma}\right),
$$

with $F_{k, \text { loc }}^{\prime}=\tilde{F}_{k, \text { loc }}+\tilde{F}_{k, \text { loc }}$, and with $\tilde{\sigma}_{1}$ summed over subsets of the index set for $\sigma$ on the left-hand side.

We now fix $\tilde{\sigma}_{1}, S_{4}$, and define $\tilde{\Lambda}_{8}^{(k)}$ as follows:

$$
\tilde{\Lambda}_{8}^{(k)}=\Lambda_{8}^{(k)} \backslash \bigcup_{\sigma \notin \tilde{\sigma}_{1}} X_{\sigma} \bigcup_{\left(j, x_{j}, X\right) \in S_{4}} X .
$$

Then we define $\Lambda_{9}^{(k)}$ by deleting a collar neighborhood of width $r\left(e_{k}\right)$ from $\tilde{\Lambda}_{8}^{(k)}$. In $\Lambda_{9}^{(k)}$ we attempt to remove the characteristic functions $\chi_{\Lambda_{9}^{(k)}}$. Thus we write

$$
\chi_{A_{0}^{(k)}}=\chi_{\Lambda_{0}^{(k)} \cap A_{9}^{(k) c}} \chi_{\Lambda_{9}^{(k)}},
$$

and for each type of characteristic function in $\chi_{\Lambda_{9}^{(k)}}$ we expand $\chi=1-\chi^{c}$, as follows:

$$
\prod_{x \in A_{9}^{(k)}} \chi_{x}=\sum_{S_{x} \subset A_{9}^{(k)}} \prod_{x \in S_{x}}\left(-\chi_{x}^{c}\right) \text {. }
$$

We have similar sums over $S_{y} \subset \Lambda_{9}^{(k) \prime}, S_{b} \subset \Lambda_{9}^{(k) *}, S_{p} \subset \Lambda_{9}^{(k) * *}$, and we define $\tilde{\Lambda}_{9}^{(k)}$ as the union of all $r\left(e_{k}\right)$-cubes in $\Lambda_{9}^{(k)}$, none of whose points are in $S_{x}$, or in bonds, plaquettes, or blocks in $S_{b}, S_{p}, S_{y}$. The characteristic function expansion can now be written as

$$
\begin{gathered}
\chi_{\Lambda_{9}^{(k)}}=\sum_{\tilde{A}_{9}^{(k)}} \zeta_{\tilde{\Lambda}_{9}^{(k) c}}^{\prime(k)}, \\
\zeta_{\tilde{\Lambda}_{9}^{(k) c}=} \sum_{\left\{S_{x}, S_{y}, S_{b}, S_{p}\right\} \text { compatible with } \tilde{A}_{9}^{(k) c}, A_{9}^{(k)}} \prod_{x \in S_{x}}\left(-\chi_{x}^{c}\right) \\
\times \prod_{y \in S_{y}}\left(-\chi_{y}^{c}\right) \prod_{b \in S_{b}}\left(-\chi_{b}^{c}\right) \prod_{p \in S_{p}}\left(-\chi_{p}^{c}\right) .
\end{gathered}
$$

Finally we define $\Lambda_{10}^{(k)}$ by deleting a collar neighborhood from $\tilde{\Lambda}_{9}^{(k)}$.

These expansions complicate our expression for $\varrho_{k+1}^{L}(v, \psi)$ in (5.9.6), however the integral in $\Lambda_{10}^{(k)}$ is quite simple now. It involves a small, local, polynomial interaction $V^{(k)}$ modifying a Gaussian integral in $\phi^{(k)}, A^{(k)}$. The inverse covariance is local and bounded from above and from below. The characteristic functions $\chi^{\prime}$ are simple functions of $\phi^{(k)}, A^{(k)}$ keeping them bounded. The observable is a product of polynomial pieces given by low-order perturbation theory. Large field and nonperturbative effects have been separated out.

\subsection{Conditional Integration}

We exploit the simple structure in $\Lambda_{10}^{(k)}$ by doing the integrals there with conditioning on $\Lambda_{10}^{(k) c}, \Lambda_{10}^{(k) c * c}$. The formula we use is a generalization of the following identity for scalar fields:

$$
\begin{aligned}
\left.\left.\int d \phi\right|_{\Lambda^{c}} F\left(\left.\phi\right|_{\Lambda^{c}}\right) \int d \phi\right|_{\Lambda} e^{-\left\langle A^{c} \phi, A \Lambda \phi\right\rangle} e^{-1 / 2\left\langle\phi, A_{\Lambda} \phi\right\rangle} G(\phi) \\
=\left.\left.\int d \phi\right|_{\Lambda^{c}} F\left(\left.\phi\right|_{\Lambda^{c}}\right) \int d \phi\right|_{\Lambda} e^{-\left\langle\Lambda^{c} \phi, A \Lambda \phi\right\rangle} e^{-1 / 2\left\langle\phi A_{\Lambda} \phi\right\rangle} \\
\quad \times \frac{\left.\int d \phi\right|_{\Lambda} G(\phi) e^{-1 / 2\left\langle\phi, A_{\Lambda} \phi\right\rangle} e^{-\left\langle\Lambda^{c} \phi, A \Lambda \phi\right\rangle}}{\left.\int d \phi\right|_{\Lambda} e^{-1 / 2\left\langle\phi, A_{\Lambda} \phi\right\rangle} e^{-\left\langle\Lambda^{c}, A \Lambda \phi\right\rangle}} \\
=\left.\left(\left.\int d \phi\right|_{\Lambda} e^{-1 / 2\left\langle\phi, A_{\Lambda} \phi\right\rangle}\right) \int d \phi\right|_{\Lambda^{c}} F\left(\left.\phi\right|_{A^{c}}\right) e^{1 / 2\left\langle\Lambda^{c} \phi, A A_{\Lambda}{ }^{1} A \Lambda^{c} \phi\right\rangle} \\
\quad \times-\left.\frac{1}{\mathscr{N}} \int d \phi\right|_{\Lambda} G(\phi) e^{-1 / 2\left\langle\phi, A_{\Lambda} \phi\right\rangle} e^{-\left\langle A^{c} \phi, A \Lambda \phi\right\rangle} .
\end{aligned}
$$


Here $\mathscr{N}$ is equal to the last integral, without $G(\phi)$. Thus in our expression for $\varrho_{k+1}^{L}(v, \psi)$, we have now an "exterior" integral over $u^{(k)}, \phi^{(k)}$ in $\Lambda_{10}^{(k) c}$, whose Gaussian piece has been replaced by

$$
\begin{aligned}
& \delta_{\mathrm{Ax}, \Lambda_{10}^{(k) ' c}}\left(u^{(k)}\right) \delta_{\Lambda_{1}^{(k) * *} \cap A_{10}^{(k) ' c *}}\left(\frac{e_{k}}{2 \pi} Q A^{(k)}\right) \\
& \times \exp \left[-\frac{1}{2}\left\langle\Lambda_{1}^{(k) * * \partial} \partial \Lambda_{10}^{(k) c *} A^{(k)}, \sigma_{k, \operatorname{loc}} \Lambda_{1}^{(k) * * \partial} \partial \Lambda_{10}^{(k) c} A^{(k)}\right\rangle\right. \\
& -\frac{1}{2}\left\langle\left(\Lambda_{8}^{(k-1)^{\prime}} \cap \Lambda_{10}^{(k) c}\right) \phi^{(k)},\left(\Delta_{k, \operatorname{loc}}\left(\tilde{u}_{k+1}\right)+a L^{-2} P\left(\tilde{u}_{k+1}\right)\right)\left(\Lambda_{8}^{(k-1)^{\prime}} \cap \Lambda_{10}^{(k) c}\right) \phi^{(k)}\right\rangle \\
& +\frac{1}{2}\left\langle\Lambda_{10}^{(k) c} \phi^{(k)}, \Delta_{k, \operatorname{loc}}\left(u_{k+1}\right) C_{\Lambda_{10}^{(k)}}^{(k)}\left(u_{k+1}\right) \Delta_{k, \text { loc }}\left(u_{k+1}\right) \Lambda_{10}^{(k) c} \phi^{(k)}\right\rangle \\
& -\frac{1}{2}\left\langle\Lambda_{10}^{(k) c *} A^{(k)}, L^{-1} Q^{*} Q^{s} \Lambda_{10}^{(k) c * c} \partial^{*} \sigma_{k, 10 c} \partial\left(L^{-1} \Lambda_{10}^{(k) c * c} Q^{s *} Q-2\right) \Lambda_{10}^{(k) c *} A^{(k)}\right\rangle \\
& +\frac{1}{2}\left\langle\Lambda_{10}^{(k) c *} A^{(k)},\left(I-L^{-1} Q^{*} Q^{s} \Lambda_{10}^{(k) c * c}\right) \partial^{*} \sigma_{k, \operatorname{loc}} \partial C_{\Lambda_{10}^{(k) c * c}}^{(k)} \partial^{*} \sigma_{k, 10 c} \partial\right. \\
& \left.\left.\times\left(I-L^{-1} \Lambda_{10}^{(k) c * c} Q^{s *} Q\right) \Lambda_{10}^{(k) c *} A^{(k)}\right\rangle\right] Z_{\Lambda_{10}^{(k) c * c}}^{(k)} Z_{\Lambda_{10}^{(k)}}^{(k)}\left(u_{k+1}\right) .
\end{aligned}
$$

The "interior" integral is

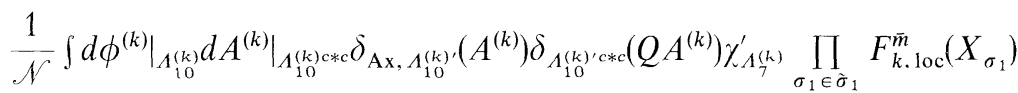

$$
\begin{aligned}
& \times \exp \left[-\frac{1}{2}\left\langle\Lambda_{10}^{(k)} \phi^{(k)},\left(\Lambda_{k \cdot 1 \mathrm{oc}}\left(u_{k+1}\right)+a L^{-2} P\left(u_{k+1}\right)\right)\left(\Lambda_{10}^{(k)}+2 \Lambda_{10}^{(k) c}\right) \phi^{(k)}\right\rangle\right. \\
& -\frac{1}{2}\left\langle\Lambda_{10}^{(k) c * c} A^{(k)}, \partial^{*} \sigma_{k, 10 c} \partial\left(\Lambda_{10}^{(k) c * c}+2 \Lambda_{10}^{(k) c *}\right) A^{(k)}\right\rangle \\
& \text { - } \left.V^{(k)}\left(\Lambda_{8}^{(k)}, u_{k+1}, A^{(k)}, \phi^{(k)}\right)\right] \text {. }
\end{aligned}
$$

Here $\mathscr{N}$ is defined by the last integral, but without $\chi_{\Lambda^{(k)}}^{\prime}, F_{k . \text { loc }}^{\bar{m}}$, or $V^{(k)}$.

Let us describe more carefully the calculations leading to (5.12.1). The third form, together with $Z_{\Lambda_{10}^{(k)}}^{(k)}\left(u_{k+1}\right)$, is a calculation of

$$
\left.\int d \phi^{(k)}\right|_{\Lambda_{10}^{(k)}} \exp \left[-\frac{1}{2}\left\langle\Lambda_{10}^{(k)} \phi^{(k)},\left(\Delta_{k, \operatorname{loc}}\left(u_{k+1}\right)+a L^{-2} P\left(u_{k+1}\right)\right)\left(\Lambda_{10}^{(k)}+2 \Lambda_{10}^{(k) c}\right) \phi^{(k)}\right\rangle\right] .
$$

The 4-th and 5-th forms, with $Z_{\Lambda_{10}^{(k) c * c}}^{(k)}$, are a calculation of

$$
\begin{aligned}
& \left.\int d A^{(k)}\right|_{\Lambda_{10}^{(k) c * c}} \delta_{\mathrm{Ax}, \Lambda_{10}^{(k)},}\left(A^{(k)}\right) \delta_{\left.\Lambda_{10}^{(k)}\right) * * c}\left(Q A^{(k)}\right)\left(e_{k} / 2 \pi\right)^{\left\|A_{10}^{(k) c * c \|}\right\|} \\
& \times \exp \left[-\frac{1}{2}\left\langle\Lambda_{10}^{(k) c * c} A^{(k)}, \partial^{*} \sigma_{k, 10 c} \partial\left(\Lambda_{10}^{(k) c * c}+2 \Lambda_{10}^{(k) c *}\right) A^{(k)}\right\rangle\right] .
\end{aligned}
$$

The factors $e_{k} / 2 \pi$ come from the replacement of $d u^{(k)}$ with $d A^{(k)}$ for the free variables; for the constrained variables the replacement is compensated by a removal of the $e_{k} / 2 \pi$ factor from the $\delta$-functions, see (4.6)-(4.8).

We calculate $(5.12 .3)$ by means of a translation

$$
A^{(k)}=A^{(k) \prime}-\Lambda_{10}^{(k) c * c} Q^{s *} Q \Lambda_{10}^{(k) c *} A^{(k)},
$$

which removes the dependence on $\Lambda_{10}^{(k) c *} A^{(k)}$ in the $\delta$-functions. In fact, $\delta_{\mathrm{Ax}}, \Lambda_{10}^{(h)},\left(A^{(k)}\right)$

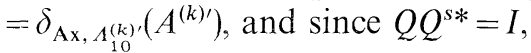

$$
\delta_{\left.\Lambda_{10}^{(k)}\right)^{\prime} * * c}\left(Q A^{(k)}\right)=\delta_{\Lambda_{10}^{(k) \prime} c * c}\left(Q \Lambda_{10}^{(k) c * c} A^{(k)^{\prime}}\right) .
$$

The fourth quadratic form above is obtained by collecting the terms in the exponential quadratic in $\Lambda_{10}^{(k) c *} A^{(k)}$. There remains a linear form

$$
\left\langle\Lambda_{10}^{(k) c * c} A^{(k) \prime}, \partial^{*} \sigma_{k .10 c} \partial\left(I-\Lambda_{10}^{(k) c * c} Q^{s *} Q\right) \Lambda_{10}^{(k) c *} A^{(k)}\right\rangle,
$$


whose expectation in the Gaussian

$$
\exp \left(-\frac{1}{2}\left\langle\Lambda_{10}^{(k) c * c} A^{(k) \prime}, \partial^{*} \sigma_{k, 10 c} \partial \Lambda_{10}^{(k) c * c} A^{(k) \prime}\right\rangle\right)
$$

gives rise to the fifth form.

We remove the nonlocality in the third and fifth quadratic forms with random walk expansions for $C_{\Lambda_{10}^{(k)}}^{(k)}\left(u_{k+1}\right)$ and for $C_{A_{10}^{(k) * * c}}^{(k)}$ as in (2.45) and (2.48). These obey the usual estimates. We denote the first and second quadratic forms by $\mathscr{Q}_{7}$, and the other three [with $C_{\Lambda_{10}^{(k)}}^{(k)}\left(u_{k+1}\right), C_{\Lambda_{10}^{(k) c * c}}^{(k)}$ replaced with $C_{\Lambda_{10}^{(k)}(k)}^{(k)}$ loc $\left(u_{k+1}\right), C_{\Lambda_{10}^{(k) c * c}, \text { loc }}^{(k)}$ ] by $\mathscr{2}_{8}$. Altogether the exponential in $(5.12 .1)$ has been written as

$$
\exp \left[-\mathscr{2}_{7}-\mathscr{Q}_{8}-\sum_{X} W_{5}^{(k)}(X)\right]
$$

Here $W_{5}^{(k)}(X)$ contains the terms with $C_{A_{10}^{(k)}, X}^{(k)}\left(u_{k+1}\right)$ or with $C_{A_{10}^{(k) c * c}, X}^{(k)}$, and satisfies $\left|W_{5}^{(k)}(X)\right| \leqq e^{-c r\left(e_{k}\right)|X|}$. The quadratic forms in $\mathscr{Z}_{7}$ and $\mathscr{Q}_{8}$ are localized near $\Lambda_{10}^{(k) c}$.

We make the same translation (5.12.4) in both numerator and denominator of the normalized integral in $\Lambda_{10}^{(k)}$. Terms quadratic in $\Lambda_{10}^{(k) c^{*}} A^{(k)}$ cancel, but we still have the linear forms (5.12.5) and $\left\langle\Lambda_{10}^{(k)} \phi^{(k)}, \Lambda_{k, 10 c}\left(u_{k+1}\right) \Lambda_{10}^{(k) c} \phi^{(k)}\right\rangle$ as in our last calculation. We remove most of these forms with localized translations

$$
\begin{gathered}
A^{(k) \prime}=A^{(k) \prime \prime}-C_{\Lambda_{10}^{(k) * c}, \operatorname{loc}}^{(k)} \partial^{*} \sigma_{k, \operatorname{loc}} \partial\left(I-L^{-1} \Lambda_{10}^{(k) c * c} Q^{s *} Q\right) \Lambda_{10}^{(k) c *} A^{(k)}, \\
\phi^{(k)}=\phi^{(k) \prime \prime}-C_{\Lambda_{10}^{(k)}, \operatorname{loc}}^{(k)}\left(u_{k+1}\right) \Lambda_{k, \operatorname{loc}}\left(u_{k+1}\right) \Lambda_{10}^{(k) c} \phi^{(k)} .
\end{gathered}
$$

Terms quadratic in $A_{10}^{(k) c *} A^{(k)}$ or $A_{10}^{(k) c} \phi^{(k)}$ cancel as before, leaving the following integral:

$$
\int d \mu_{\Lambda_{10}^{(k)}}^{(k)}\left(A^{(k) \prime \prime}, \phi^{(k) \prime \prime}\right) \chi_{\Lambda}^{\prime(k)} \prod_{\sigma_{1} \in \tilde{\sigma}_{1}} F_{k, \operatorname{loc}}^{\bar{m}}\left(X_{\sigma_{1}}\right) e^{-V^{(k)}\left(\Lambda_{8}^{(k)}, u_{k+1} \cdot A^{(k)} \cdot \phi^{(k)}\right)} .
$$

Here $d \mu_{\Lambda_{10}^{(k)}}^{(k)}$ is an uncentered, normalized Gaussian measure,

$$
\begin{aligned}
& d \mu_{\Lambda_{10}^{(k)}}^{(k)}\left(A^{(k) \prime \prime}, \phi^{(k) \prime \prime}\right) \\
& =\left.\left.\frac{1}{\mathscr{N}} d A^{(k) \prime \prime}\right|_{\Lambda_{10}^{(k) c * c}} d \phi^{(k) \prime \prime}\right|_{\substack{\Lambda_{10}^{(k)} \\
\text { (k) }}} \delta_{\mathrm{Ax}, \Lambda_{10}^{(k)}}\left(A^{(k) \prime \prime}\right) \delta_{\Lambda_{10}^{(h) \prime c * c}}\left(Q \Lambda_{10}^{(k) c * c} A^{(k) \prime \prime}\right) \\
& \times \exp \left[-\frac{1}{2}\left\langle\Lambda_{10}^{(k) c * c} A^{(k) \prime \prime}, \partial^{*} \sigma_{k \cdot \operatorname{loc}} \partial \Lambda_{10}^{(k) c * c} A^{(k) \prime \prime}\right\rangle\right. \\
& -\frac{1}{2}\left\langle\Lambda_{10}^{(k)} \phi^{(k)},\left(\Delta_{k \cdot \operatorname{loc}}\left(u_{k+1}\right)+a L^{-2} P\left(u_{k+1}\right)\right) \Lambda_{10}^{(k)} \phi^{(k)}\right\rangle \\
& -\left\langle\Lambda_{10}^{(k) c * c} A^{(k) \prime \prime},\left(I-\partial^{*} \sigma_{k, \text { loc }} \partial C_{\Lambda_{10}^{(k) c * c}, \text { loc }}^{(k)}\right) \partial^{*} \sigma_{k, \text { loc }} \partial\left(I-L^{-1} \Lambda_{10}^{(k) c * c} Q^{s *} Q\right) \Lambda_{10}^{(k) c *} A^{(k)}\right\rangle \\
& -\left\langle\Lambda_{10}^{(k)} \phi^{(k) \prime \prime},\left(I-\left(\Delta_{k \cdot 10 c}\left(u_{k+1}\right)+a L^{-2} P\left(u_{k+1}\right)\right)\right.\right. \\
& \left.\left.\left.\times C_{\Lambda_{10}^{(k)}(\operatorname{loc}}^{\left(u_{k}\right)}\left(u_{k+1}\right)\right) \Lambda_{k, \operatorname{loc}}\left(u_{k+1}\right) \Lambda_{10}^{(k) c} \phi^{(k)}\right\rangle\right] \text {. }
\end{aligned}
$$

This measure has covariances $C_{A_{(1)}^{(k) c * c}}^{(k)}, C_{A_{10}^{(k)}}^{(k)}\left(u_{k+1}\right)$, and nonzero means reflecting the terms linear in $\Lambda_{10}^{(k)} \phi^{(k) \prime \prime}$ or $\Lambda_{10}^{(k) c * c} A^{(k) \prime \prime \prime}$. 
After the conditioning our density assumes the following form:

$$
\begin{aligned}
& \varrho_{k+1}^{L}(v, \psi) \\
& =\left.\left.\sum_{\left\{X_{\omega\}}\right\}} \sum_{\Lambda_{0}^{(k)}} \sum_{S_{4}} \sum_{\dot{\sigma}_{1}} \sum_{\dot{\Lambda}_{9}^{(k)}} \int \prod_{j=0}^{k} d u^{(i)}\right|_{\Lambda_{10}^{(j) c *}} d \phi^{(k)}\right|_{\Lambda_{10}^{(k) c}} \delta_{\mathrm{Ax}, \Lambda_{10}^{(k), c}}\left(u^{(k)}\right)
\end{aligned}
$$

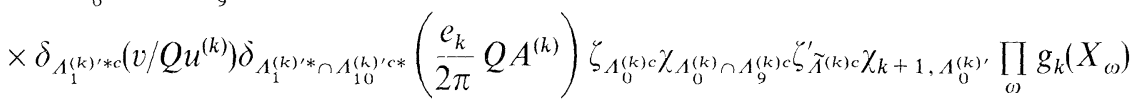

$$
\begin{aligned}
& \times \prod_{\sigma \notin \tilde{\sigma}_{1}} F_{(k), \operatorname{loc}}^{\prime}\left(X_{\sigma}\right) \prod_{\left(j, x_{j}, X\right) \in S_{4}}\left(e^{-W_{4, j}^{(h)}\left(x_{J}, X\right)}-1\right) \prod_{j=0}^{k}\left[Z_{A_{10}^{(j)}(j) c * c}^{(j)} Z_{A_{10}^{(j)}}^{(j)}\left(u_{k+1}\right)\right]
\end{aligned}
$$

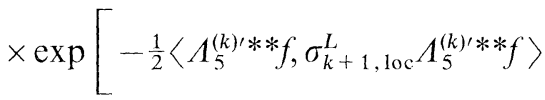

$$
\begin{aligned}
& -\frac{1}{2}\left\langle\Lambda_{8}^{(k) \prime} \psi, \Delta_{k+1 . \operatorname{loc}}^{L}\left(u_{k+1}\right) \Lambda_{8}^{(k) \prime} \psi\right\rangle-\sum_{i=1}^{8} \mathscr{Q}_{i} \\
& -\mathscr{E}_{k}-E^{(k)}-\mathscr{P}_{h \cdot 1 \mathrm{loc}}\left(\Lambda_{8}^{k+1}, \Lambda_{8}^{(k) c}, \tilde{u}_{k+1}\right)-R^{(k)}\left(\Lambda_{8}^{(k) c}, u_{k+1}, \theta_{k} H_{k, 1 \mathrm{loc}} A^{(k)}\right) \\
& \left.-Q^{(k)}\left(\Lambda_{8}^{(k) c}, u_{k+1}, \theta_{k} H_{k \cdot 1 \mathrm{loc}} A^{(k)}\right)\right] \int d \mu_{\Lambda_{10}^{(k)}}^{(k)}\left(A^{(k) \prime \prime}, \phi^{(k) \prime \prime}\right) \chi_{\Lambda_{7}^{\prime}}^{\prime(k)} \prod_{\sigma_{1} \in \tilde{\sigma}_{1}} F_{k, \operatorname{loc}}^{\bar{m}}\left(X_{\sigma_{1}}\right) \\
& \times \exp \left[-V^{(k)}\left(\Lambda_{8}^{(k)}, u_{k+1}, A^{(k)}, \phi^{(k)}\right)-\sum_{X} W_{5}^{(k)}(X)\right] .
\end{aligned}
$$

The next two sections will focus on deriving a cluster expansion for the $d \mu_{\Lambda_{1}^{(k)}}^{(k)}$ integral in $(5.12 .8)$

\subsection{Decoupling of the Small Field Region}

We give a cluster expansion for the $d \mu_{\Lambda_{10}^{(k)}}^{(k)}$ integral in (5.12.8). The purpose is to remove the dependence of the small field integral on the boundary fields. The cluster expansion has two parts; Mayer expansion of the interaction, and interpolation of the covariances of $d \mu_{\Lambda_{10}^{(k)}}$.

Let us divide $\Lambda_{7}^{(k)}$ into its elementary $r\left(e_{k}\right)$-cubes $\square^{(x)}$. We assign to $\square^{(\alpha)}$ all bonds $\left\langle x, x+e_{\mu}\right\rangle$ with $x \in \square^{(\alpha)}$. Note that $V^{(k)}\left(\Lambda_{8}^{(k)}, u_{k+1}, A^{(k)}, \phi^{(k)}\right)$ involves $\left.A^{(k)}\right|_{\Lambda_{7}^{(h) *},}$ $\left.\phi^{(k)}\right|_{A_{7}^{(k)}}$ only. Thus we localize the fields $\phi^{(k)}, A^{(k)}$ in $V^{(k)}$ by writing

$$
\phi^{(k)}=\sum_{\alpha} \square^{(\alpha)} \phi^{(k)}, \quad A^{(k)}=\sum_{\alpha} \square^{(\alpha)} A^{(k)} .
$$

We associate to any collection of localization cubes a smallest connected union of cubes containing them (call it $Y$ ). Summing over all terms in $V^{(k)}$ and over localizations giving rise to $Y$, we obtain a decomposition.

$$
V^{(k)}\left(\Lambda_{8}^{(k)}, u_{k+1}, A^{(k)}, \phi^{(k)}\right)=\sum_{Y} V^{(k)}(Y)+V_{\text {const }}^{(k)}\left(\Lambda_{8}^{(k)}\right) .
$$

The last term includes all terms independent of $A^{(k)}, \phi^{(k)}$. We have an estimate $\left|V^{(k)}(Y)\right| \leqq e^{\beta}\left(L^{k} \varepsilon / \varepsilon_{0}\right)^{1 / 4-\alpha}$. Note that $Y$ contains at most a few cubes.

Next we Mayer-expand the interaction

$$
\begin{aligned}
& \exp \left[-V^{(k)}\left(\Lambda_{8}^{(k)}, u_{k+1}, A^{(k)}, \phi^{(k)}\right)-\sum_{X} W_{5}^{(k)}(X)\right] \\
& =\sum_{S_{Y}} \sum_{S_{5}} e^{-V_{\text {const }}^{(k)}\left(A_{8}^{(k)}\right)} \prod_{Y \in S_{Y}}\left(e^{-V^{(k)}(Y)}-1\right) \prod_{X \in S_{5}}\left(e^{-W_{5}^{(k)}(X)}-1\right) .
\end{aligned}
$$


Here $S_{Y}\left(S_{5}\right)$ is the set of all Y's ( $X^{\prime}$ 's) that arise in a term in the Mayer expansion.

We decompose $\Lambda_{10}^{(k)}$ into elementary regions $\left\{\square_{i}\right\}_{i \in I}$ which are connected unions of $\square^{(\alpha)}$. Two $\square^{(\alpha)}$ are included into a $\square_{i}$ if one of the following conditions hold:

(i) They are both in some $Y, Y \in S_{Y}$,

(ii) They are both in some $X, X \in S_{5}$,

(iii) They both contain sites or bonds within $r\left(e_{k}\right)$ of some $X_{\sigma_{1}}, \sigma_{1} \in \tilde{\sigma}_{1}$.

(iv) They are both in a connected component of $\Lambda_{11}^{(k) c}$.

For the decoupling of the Gaussian measure, we interpolate the covariance with parameters $s_{i} \in[0,1], i \in I$, which turn off interactions between $\square_{i}$ and $\square_{i}^{c}$. The factors $\delta\left(\left(Q A^{(k) \prime \prime}\right)\left(b^{\prime}\right)\right)$ in the measure constitute an interaction between blocks. It is convenient to treat them directly, so we trade them for a fictitious integration $d B$, where $B$ is a field on $\Lambda_{10}^{(k) \prime c * c}$. We insert $1=\mathscr{N}^{-1} \int d B \exp (-1 / 2\langle B, B\rangle)$ and translate $A^{(k) \prime \prime}$ by $Q^{s *} B$ to obtain

$$
\begin{aligned}
& \left.\int d A^{(k) \prime \prime}\right|_{A_{10}^{(k) * * c}} \delta_{A_{10}^{(k) \prime c * c}}\left(Q A_{10}^{(k) c * c} A\right) \delta_{\mathrm{Ax}, A_{10}^{(k) *}\left(A^{(k) \prime \prime}\right) f\left(A^{(k) \prime \prime}\right)} \\
& =\mathscr{N}^{-1} \int d A^{(k) \prime \prime} d B e^{-1 / 2\langle B, B\rangle} \delta\left(Q A^{(k) \prime \prime}+Q Q^{s *} B\right) \delta_{\mathrm{Ax}}\left(A^{(k) \prime \prime}\right) f\left(A^{(k) \prime \prime}+Q^{s *} B\right) .
\end{aligned}
$$

The translation does not affect $\delta_{\Lambda x}$, and by (I.2.19) we have $Q Q^{s *}=I$. Integrating out $B$ yields

$$
\mathscr{N}^{-1} \int d A^{(k) \prime} \exp \left[-\frac{1}{2}\left\langle A^{(k) \prime \prime}, Q^{*} Q A^{(k) \prime \prime}\right\rangle\right] \delta_{\mathrm{Ax}}\left(A^{(k) \prime \prime}\right) f\left(\left(I-Q^{s *} Q\right) A^{(k) \prime}\right) .
$$

Thus we have a new quadratic form for $\Lambda_{10}^{(k) c * c} A^{(k) \prime \prime}$, namely

$$
Q^{*} Q+\left(I-Q^{*} Q^{s}\right) \partial^{*} \sigma_{k, 1 \mathrm{co}} \partial\left(I-Q^{s *} Q\right) \text {. }
$$

This is still bounded below on the subspace determined by $\delta_{\mathrm{Ax}}\left(A^{(k) \prime}\right)$ : our lower bound on $\partial^{*} \sigma_{k, \text { loc }} \partial$ implies a lower bound

$$
\left\|Q A^{(k) \prime \prime}\right\|^{2}+O(1)\left\|A^{(k) \prime \prime}-Q^{s *} Q A^{(k) \prime}\right\|^{2} \geqq O(1)\left\|A^{(k) \prime \prime}\right\|^{2} .
$$

Applying (5.13.1) to numerator and denominator of the $d \mu_{\Lambda_{10}^{(k)}}^{(k)}$-integral in (5.12.8), the $\mathcal{N}$ 's cancel, and we obtain

$$
\begin{aligned}
& \int d \mu_{\Lambda_{10}^{(k)}}^{(k)}\left(A^{(k) \prime \prime}, \phi^{(k) \prime \prime}\right) \chi_{\Lambda_{7}^{(k)}}^{\prime} \prod_{\sigma_{1} \in \tilde{\sigma}_{1}} F_{k, \operatorname{loc}}^{\bar{m}}\left(X_{\sigma_{1}}\right) \prod_{Y \in S_{Y}}\left(e^{-V^{(k)}(Y)}-1\right) \\
& \times \prod_{X \in S_{5}}\left(e^{-W_{5}^{(k)}(X)}-1\right)=\left\langle\prod_{i \in I} f\left(\square_{i}\right)\right\rangle_{\underline{1}},
\end{aligned}
$$

where $f\left(\square_{i}\right)$ is the product of all the factors under the $d \mu_{\Lambda_{10}^{(k)}}^{(k)}$ integral above that are localized in $\square_{i}$. (Factors localized in $\Lambda_{10}^{(k) c}$ are assigned to the $\square_{i}$ intersecting the corresponding component of $\Lambda_{11}^{(k) c}$.) Our construction of the $\square_{i}$ ensures no overlap of factors between different $\square_{i}$ 's. Everywhere $A^{(k) \prime}$ appears as $\left(I-Q^{s *} Q\right) A^{(k) \prime \prime}$. The expectation $\langle\cdot\rangle_{1}$ is in the measure

$$
\left.\frac{1}{\mathscr{N}^{\prime}} d \Phi\right|_{\Lambda_{10}^{(k)}} \delta_{\mathrm{Ax}, A_{10}^{(k) *}}\left(A^{(k) \prime \prime}\right) \exp \left[\frac{1}{2}\langle\Phi, \Delta \Phi\rangle+\langle\Phi, \mathscr{F}\rangle\right]
$$

We have simplified the notation by writing $\Phi=\left(A^{(k) \prime \prime}, \quad \phi^{(k) \prime}\right),\left.\quad d \Phi\right|_{\Lambda_{10}^{(h) \prime \prime}}$ $=\left.\left.d A^{(k) \prime \prime}\right|_{\Lambda_{10}^{(k) c * c}} d \phi^{(k) \prime \prime}\right|_{\Lambda_{10}^{(k)}}, \Lambda_{10}^{(k) c} \Phi=\left(\Lambda_{10}^{(k) c *} A^{(k) " \prime}, \Lambda_{10}^{(k) c} \phi^{(k) \prime \prime}\right)$, and so on. The quadratic 
and linear forms $\Delta$ and $\mathscr{F}$ are obtained in the obvious fashion from (5.12.7), replacing $\Lambda_{10}^{(k) c * c} A^{(k) \prime \prime}$ with $\left(1-Q^{s *} Q\right) \Lambda_{10}^{(k) c * c} A^{(k) \prime \prime}$. The linear form is localized near the boundary of $\Lambda_{10}^{(k)}$.

To preserve positivity and boundedness properties of the inverse covariance, we define our s-dependent inverse covariance by taking convex combinations of inverse covariances with Dirichlet boundary conditions. For an arbitrary subset $I$ of $I$ we define Dirichlet forms:

$$
\Delta_{\Gamma}=\sum_{i \in A} \square_{i} \Delta \square_{i}+\square^{c} \Delta \square^{c},
$$

where $\square^{c}=\bigcup_{i \neq \Gamma} \square_{i}$, and all operators are restricted to the subspace $A^{(k) \prime \prime}\left(\Gamma_{y, x}\right)=0$.

Next we define an operation

$$
a_{\Gamma} \Delta_{\Gamma^{\prime}}=\Delta_{\Gamma \cup \Gamma^{\prime}},
$$

and we define a quadratic form for $\underline{s}=\left\{s_{i}\right\}_{i \in I}$ :

$$
\Delta_{\underline{s}}=\prod_{i \in I}\left[\left(1-s_{i}\right) a_{i}+s_{i}\right] \Delta=\sum_{\Gamma \subset I} \prod_{i \in \Gamma}\left(1-s_{i}\right) \prod_{i \in I \backslash \Gamma} s_{i} \Delta_{\Gamma} .
$$

Note that by resumming the expansion above and using the fact that for $i^{\prime \prime} \neq i$ or $i^{\prime}$, or for $i=i^{\prime}, \square_{i}\left(a_{\square^{\prime \prime}}, 4\right) \square_{i^{\prime}}=\square_{i} \Delta \square_{i^{\prime}}$, we obtain that

$$
\begin{gathered}
\square_{i} \Delta_{\underline{\underline{S}}} \square_{i^{\prime}}=s_{i} s_{i^{\prime}} \square_{i} \Delta \square_{i^{\prime}}, \quad i^{\prime} \neq i \\
\square{ }_{i} \Delta_{\underline{s}} \square_{i}=\square_{\imath} \Delta \square_{i} .
\end{gathered}
$$

Using the theorem on unit lattice operators in [6], we can invert this operator to yield an exponentially decaying covariance $C_{\underline{s}}=\left(-\Delta_{s}\right)^{-1}$.

To give our expansion, we use the fundamental theorem of calculus to write

$$
\left\langle\prod_{i \in i} f\left(\square_{i}\right)\right\rangle_{\underline{1}}=\sum_{\Gamma \subset I} \int d \underline{s}_{\Gamma} \frac{\partial}{\partial s_{I}}\left\langle\prod_{i \in I} f\left(\square_{i}\right)\right\rangle_{\underline{s}_{r}} .
$$

Here $\underline{s}_{\Gamma}$ specifies $s_{i}=0$ for $i \notin \Gamma, d \underline{s}_{\Gamma}=\prod_{i \in \Gamma} d s_{i}, \partial / \partial s_{\Gamma}=\prod_{i \in \Gamma} d / d s_{i}$, and $\langle\cdot\rangle_{\underline{s}_{\Gamma}}$ is the expectation with quadratic form $\Delta_{\underline{S}_{\Gamma}}$ instead of $\Delta$. To calculate the $s$-derivatives, note that the first derivative produces a term

$$
\left\langle\sum_{j \neq i} s_{j}\left\langle\square_{i} \Phi, \Delta \square_{j} \Phi\right\rangle ; \prod_{i \in I} f\left(\square_{i}\right)\right\rangle_{\underline{s}_{I}} .
$$

Subsequent derivatives either hit factors $s_{j}$ already pulled down or bring new terms down with new truncations. After all derivatives are performed, we set the remaining $s_{j}$ to zero, so only terms with no $s_{j}$ multiplying them survive. The result is

$$
\left\langle\prod_{l \in I} f\left(\square_{i}\right)\right\rangle_{\underline{1}}=\sum_{\Gamma \subset I} \int d \underline{s}_{\Gamma} \sum_{\substack{\text { pairings } \\ p_{\gamma}=\left\{i_{\gamma}, j_{\gamma}\right\}}}\left\langle\prod_{\gamma=1}^{|\Gamma| / 2}\left[\left\langle\square_{i_{\gamma}} \Phi, \Delta \square_{j_{\gamma}} \Phi\right\rangle ;\right] \prod_{i \in I} f\left(\square_{i}\right)\right\rangle_{\underline{s}_{\Gamma}} .
$$

Recall that we have a linear term in the measure, $e^{\langle\Phi, \mathscr{F}\rangle}$. With this term, integration by parts replaces $\Phi$ by $C_{\underline{\underline{s}}}(\delta / \delta \Phi)+C_{\underline{\underline{s}}} \mathscr{\mathcal { F }}$ (see Eqs. (12.2), (12.3) of [3] where a similar expansion is used). We integrate by parts all fields appearing in this formula. Each $\Phi$ contracts through a $C_{\underline{s}}$ to another $\Phi$, to an $f\left(\square_{i}\right)$, or to $\mathscr{F}$. If a 
closed loop forms, or if a train of covariances beginning and ending in $\mathscr{F}$ forms, then the term disappears with truncation. Thus we have only trains beginning with a $\delta / \delta \Phi$ and ending in either $\delta / \delta \Phi$ or $\mathscr{F}$. The sum over pairings and the sum over ways of arranging the contractions combine into a sum over walks $\left.\left\{\omega_{\alpha}\right\}_{\alpha \in \pi}, \alpha\right)=\left(i_{1}\right.$, $\left.i_{2}, \ldots, i_{|\omega|}\right)$ involving the sites in $\alpha$, an element of a partition $\pi$ of $\Gamma$. Thus letting $\mathscr{P}(\Gamma)$ denote the partitions of $\Gamma$, we have

$$
\begin{aligned}
\left\langle\prod_{i \in I} f\left(\square_{l}\right)\right\rangle_{\underline{1}} & =\sum_{\Gamma \subset I} \int d \underline{s}_{\Gamma} \sum_{\pi \in \mathscr{P}(\Gamma)}\left\langle\prod _ { x \in \pi } \left[\sum _ { \omega ( x ) } \left\langle\frac{\delta}{\delta \Phi}, C_{\underline{s}} \square_{i_{1}} \Delta \square_{1_{2}} C_{\underline{s}} \square_{13} \Delta \square_{14}\right.\right.\right. \\
& \left.\left.\left.\ldots \Delta \square_{i_{|(\omega)(x)|}} C_{\underline{s}}\left(\frac{1}{2} \frac{\delta}{\delta \Phi}+\mathscr{F}\right)\right\rangle\right] \prod_{i \in I} f\left(\square_{i}\right)\right\rangle_{\underline{\underline{S}}}
\end{aligned}
$$

The $1 / 2$ for the $\delta^{2} / \delta \Phi^{2}$ term compensates for the fact that we count a walk as being different from its reverse. The combinatoric structure of (5.13.3) is very similar to that of the GJS cluster expansion [9].

Let us examine the factorization properties of this expansion. The form $\Delta$ has a range less than $1 / 2 r\left(e_{k}\right)$. The $f\left(\square_{i}\right)$ do not couple different $\square$. Hence only adjacent $\square$ with $s_{i} \neq 0$ interact in the above formula. Thus our expression for $d / d \underline{s}_{I}\left\langle\prod_{i \in I} f\left(\square_{i}\right)\right\rangle_{\underline{\underline{g}}}$ factorizes over the connected components of $\Gamma$. (Here we say that $\square_{i}$ is connected to $\square_{t}$, if they abut on a hypersurface of any dimension.) The expression also factorizes over the $\square_{i} ; i \in I \backslash \Gamma$. Call the factorization regions clusters.

It is worth mentioning here that only clusters intersecting $A_{11}^{(k) c}$ have any dependence on $\Lambda_{10}^{(k) c} \phi^{(k)}, \Lambda_{10}^{(k) c *} A^{(k)}$. This is because $\mathscr{F}, A^{(k)}-A^{(k) \prime \prime}, \phi^{(k)}-\phi^{(k) \prime \prime}$ are nonzero only in $A_{11}^{(k) c}$. Thus we have finally decoupled clusters that do not intersect $\Lambda_{11}^{(k) c}$ from the large field regions - at least in so far as the fields $u^{(k)}, \phi^{(k)}$ are concerned. There is still dependence on the block fields $v, \psi$ which have yet to be integrated over and decoupled. We denote by $\Lambda_{12}^{(k)}$ the set of sites in clusters not intersecting $A_{11}^{(k) c}$.

We give now the expression for the polymer activities of this expansion. Given some region $X$, a union of $\square$, we sum $\Gamma$ over all subsets of $\left\{i \in I: \square{ }_{i} \subset X\right\}$, such that $X$ is a single cluster. Writing

$$
C_{\omega}=C_{\underline{s}} \square_{i_{1}} \Delta \square_{i_{2}} C_{\underline{s}} \square_{i_{3}} \ldots \square_{i_{|\omega|}} C_{\underline{s}},
$$

we have

$$
g_{1}(X)=\sum_{\Gamma} \int d \underline{s}_{\Gamma} \sum_{\pi \in \mathscr{P}(I)}\left\langle\prod_{x \in \pi}\left[\sum_{\omega(\alpha)}\left\langle\frac{\delta}{\delta \Phi}, C_{\omega(\alpha)}\left(\frac{1}{2} \frac{\delta}{\delta \Phi}+\mathscr{F}\right)\right\rangle\right] \prod_{\square_{1} \subset X} f\left(\square_{1}\right)\right\rangle_{\underline{s}_{r} \cdot X} .
$$

Here $\underline{s}=\left\{s_{i}: \square_{i} \subset X\right\}$, and $\langle\cdot\rangle_{\underline{I}_{I}, X}$ is defined by integrating over the fields in $X$ only.

We obtain the following expressions for the $d \mu_{A_{10}^{(k)}}^{(k)}$-integral in (5.12.8):

$$
\sum_{S_{Y}} \sum_{S_{5}} e^{-V_{\text {const }}^{(k)}\left(A_{8}^{(k)}\right)} \sum_{\left\{X_{\alpha}\right\} \text { filling } A_{10}^{(h)}} \prod_{\alpha} g_{1}\left(X_{\alpha}\right)=e^{-V_{\text {const }}^{(k)}\left(A_{8}^{(k)}\right)} \sum_{\left\{X_{\alpha\}} \prod_{\alpha}\right.} g_{2}\left(X_{\alpha}\right) .
$$

Here $g_{2}\left(X_{\alpha}\right)$ is obtained by summing over $S_{Y}, S_{5}$ compatible with $X_{\alpha}$ (each $Y, X$ is contained in $X_{\alpha}$ or the corresponding component of $\left.A_{11}^{(k) c}\right)$ :

$$
g_{2}\left(X_{\alpha}\right)=\sum_{S_{Y}, S_{5} \text { compatible with } X_{\alpha}} g_{1}\left(X_{\alpha}\right) .
$$


Let us estimate $g_{2}\left(X_{\alpha}\right)$ now. Each time some cubes are joined into one $\square_{i}$ by an $e^{-V^{(k)}(Y)}-1$ or an $e^{-W_{5}^{(k)}(X)}-1$, we get a factor $e^{\beta}\left(L^{k} \varepsilon / \varepsilon_{0}\right)^{1 / 4-\alpha}$ or $e^{-c r\left(e_{k}\right)}$. Each time some $\square_{i}$ 's are joined, we have $s$-derivatives, which produce functional derivatives, chains of covariances $C_{\omega(\alpha)}$, and factors $\mathscr{F}=O\left(e^{-c r\left(e_{k}\right)}\right)$. Functional derivatives hitting $\chi$-factors farther than $1 / 2 r\left(e_{k}\right)$ from $\Lambda_{10}^{(k) c}$ produce factors $e^{-c p\left(e_{k}\right)^{2}}$ after integrating with respect to $A^{(k) \prime \prime}, \phi^{(k) \prime}$. These derivatives are supported at $\left|A^{(k) \prime}\right|$ $\geqq c p\left(e_{k}\right)$ or $\left|\phi^{(k) \prime \prime}\right| \geqq c p\left(e_{k}\right)$ (here we use the fact that the translation vanishes). Thus we can use the arguments at the end of Sect. 14 in [3] to extract the factors $e^{-c p\left(e_{k}\right)^{2}}$ from the Gaussian measure. Functional derivatives hitting $e^{-V^{(k)}(Y)}$ yield factors $e^{\beta}\left(L^{k} \varepsilon / \varepsilon_{0}\right)^{1 / 4-\alpha}$. Functional derivatives hitting $\chi^{\prime}$-factors within $\frac{1}{2} r\left(e_{k}\right)$ of $\Lambda_{10}^{(k) c}$ are connected through $C_{\omega(\alpha)}$ to $\Lambda_{11}^{(k)}$, so we get small factors $e^{-\operatorname{cr}\left(e_{k}\right)}$ from the exponential decay of the operators $C_{s}$ and $\Delta$ in $C_{\omega(\alpha)}$. Altogether we have small factors at each end of $C_{\omega(\alpha)}$ (except for contractions to $F_{k, \text { loc }}^{\bar{m}}\left(X_{\sigma_{1}}\right)$.) If the walk $\omega(\alpha)$ wanders through more than a few cubes, we begin to pickup factors $e^{-\operatorname{cr}\left(e_{k}\right)}$. These control the sum over walks and partitions, and the factorials, as in [9]. (Factorials can be produced when many functional derivatives hit the same object, for example a characteristic function.)

Altogether, we typically get at least a small power of $e^{\beta}\left(L^{k} \varepsilon / \varepsilon_{0}\right)^{1 / 4-\alpha}$ in every cube of $X_{\alpha}$. The exceptions are when cubes are in a component of $\Lambda_{11}^{(k) c}$, when they support some $F_{k, \text { loc }}^{\bar{m}}\left(X_{\sigma_{1}}\right)$, or when $X_{\alpha}$ is a single cube. We must allow for divergent factors such as $\left(L^{k} \varepsilon\right)^{-\bar{m}}$ at $F_{k, \text { loc }}^{\bar{m}}\left(X_{\sigma_{1}}\right)$, where $\tilde{m}$ depends on $F$. Estimating the sums over $S_{v}, S_{5}$, and the sums in the cluster expansion leads to combinatoric factors

$$
\exp \left(\left(e^{\beta}\left(L^{k} \varepsilon / \varepsilon_{0}\right)^{1 / 4-\alpha}\right)^{\beta^{\prime}}\left|X_{\alpha}\right|\right), \quad \beta^{\prime}>0 .
$$

Such factors are easily beaten by the small factors described above for nonexceptional cubes. For the cubes in $\Lambda_{11}^{(k) c}$ or for a single cube, we have to include the proper volume factor in our final estimate.

In sum, we have the following bound or $g_{2}\left(X_{\alpha}\right)$ :

$$
\begin{aligned}
\left|g_{2}\left(X_{\alpha}\right)\right| \leqq \exp \left[\left(e^{\beta}\left(L^{k} \varepsilon / \varepsilon_{0}\right)^{1 / 4-\alpha}\right)^{\beta^{\prime}}\left(\left|X_{\alpha} \cap \Lambda_{11}^{(k) c}\right|+1\right)\right] & \\
& \times \prod_{\sigma_{1}}\left(L^{k} \varepsilon\right)^{-\tilde{m}\left(\sigma_{1}\right)}\left(e^{\beta}\left(L^{k} \varepsilon / \varepsilon_{0}\right)^{1 / 4-\alpha}\right)^{\beta^{\prime} \mid X_{\alpha} \backslash \Lambda_{11}^{(h) c \mid}} .
\end{aligned}
$$

The product over $\sigma_{1}$ runs over $\sigma_{1} \in \tilde{\sigma}_{1}$ such that $X_{\sigma_{1}} \subset X_{\alpha}$ or $X_{\sigma_{1}}$ is in a component of $\Lambda_{11}^{(k) c}$ overlapping $X_{\alpha}$. If $\left|X_{\alpha}\right|=1$, with no $F_{k, \text { loc }}^{\bar{m}}$-factors, then we have the more precise bound $\left|g_{2}\left(X_{\alpha}\right)-1\right| \leqq e^{\beta}\left(L^{k} \varepsilon / \varepsilon_{0}\right)^{1 / 4-\alpha}$, obtained from the same estimates on the $S_{Y}, S_{5}$ sums, and from extremely small factors when a $\chi^{\prime}$-factor is replaced by 1 .

\subsection{Resummation and Extraction of the Perturbation Expansion}

The estimates in the last section show that the basic volume dependence or pressure for our expansion is naively of the order of $\left(e^{\beta}\left(L^{k} \varepsilon / \varepsilon_{0}\right)^{1 / 4-x}\right)^{\beta^{\prime}}$. We need to do better in $\Lambda_{12}^{(k)}$, the region that has been decoupled from the large field regions. We improve our expansion in $\Lambda_{12}^{(k)}$ by computing the pressure and the expectation of $F_{k, \text { loc }}^{\bar{m}}$ as perturbation series plus remainders of the order of $\left(e^{\beta}\left(L^{k} \varepsilon / \varepsilon_{0}\right)^{1 / 4-\alpha}\right)^{\bar{n}+1}$, $\left(e^{\beta}\left(L^{k} \varepsilon / \varepsilon_{0}\right)^{1 / 4-\alpha}\right)^{\bar{m}+1}$, respectively. The remainder terms are so small that they can be treated like the large field effects and ignored in the expansion at the next scale. 
The perturbative terms exhibit renormalization cancellations, and so obey the bounds we need for the next step.

To extract the perturbative terms, we resum the decoupling and Mayer expansions in $\Lambda_{12}^{(k)}$. Note that $W_{5}^{(k)}(X) \neq 0$ only for $X$ at the boundary of $\Lambda_{10}^{(k)}$. Thus $W_{5}^{(k)}$-terms will not appear in the resummed expansion. We obtain for the expansion in (5.13.4)

$$
\sum_{\left\{X_{\alpha}\right\}} \prod_{\alpha} g_{2}\left(X_{\alpha}\right)=\sum_{\left\{X_{\alpha}\right\} \text { overlapping } \Lambda_{11}^{(k) c}} \prod_{\alpha} g_{2}\left(X_{\alpha}\right) z_{F}\left(\Lambda_{12}^{(k)}\right),
$$

where

$$
\begin{aligned}
& z_{F}\left(\Lambda_{12}^{(k)}\right)=\left\langle\chi_{\Lambda_{12}^{(k)}}^{\prime} \prod_{\sigma_{1}: X_{\sigma_{1}} \subset \Lambda_{12}^{(k)}} F_{k \cdot \operatorname{loc}}^{\bar{m}}\left(X_{\sigma_{1}}\right) e^{-\tilde{V}(k)\left(\Lambda_{12}^{(k)}\right)}\right\rangle_{\underline{1}, \Lambda_{12}^{(k)}}, \\
& \tilde{V}^{(k)}\left(\Lambda_{12}^{(k)}\right)=\sum_{Y \subset \Lambda_{12}^{(k)}} V^{(k)}(Y) .
\end{aligned}
$$

Recall that $A^{(k) \prime \prime}=A^{(k)}, \phi^{(k) \prime \prime}=\phi^{(k)}$ in $\Lambda_{12}^{(k)}$, and that $A^{(k) \prime \prime}$ has been replaced by $\left(I-Q^{s *} Q\right) A^{(k) \prime \prime}$ everywhere in the integrand.

We treat $z_{F}\left(\Lambda_{12}^{(k)}\right)$ as follows:

$$
z_{F}\left(\Lambda_{12}^{(k)}\right)=\frac{z_{F}\left(\Lambda_{12}^{(k)}\right)}{z\left(\Lambda_{12}^{(k)}\right)} \exp \left(\log z\left(\Lambda_{12}^{(k)}\right)\right),
$$

where $z\left(\Lambda_{12}^{(k)}\right)=z_{F=1}\left(\Lambda_{12}^{(k)}\right)$, and we give expansions for $z_{F} / z$ and $\log z$. The first expansion will give rise to $F_{k+1, \text { loc }}^{L}$ plus remainders, the second to $\mathscr{P}_{k+1, \text { loc }}^{L}$ plus remainders. We consider only $\log z$ for the moment.

Define $z_{t}\left(\Lambda_{12}^{(k)}\right)$ for $t \in[0,1]$ by replacing $\widetilde{V}\left(\Lambda_{12}^{(k)}\right)$ with $t \tilde{V}\left(\Lambda_{12}^{(k)}\right)$, replacing $\chi\left(c p\left(e_{k}\right)\right.$, $\left.\left(I-Q^{s *} Q\right) A^{(k)}\right)$ with $\chi\left(c p\left(t e_{k}\right),\left(I-Q^{s *} Q\right) A^{(k)}\right)$, and similarly for $\chi\left(c p\left(e_{k}\right), \phi^{(k)}\right)$. Thus the restrictions and the interactions disappear at $t=0$, at which point we have a purely Gaussian expectation.

Thus we define perturbative terms for the action,

$$
\widetilde{\mathscr{P}}_{k+1}\left(\Lambda_{12}^{(k)}\right)=\sum_{\alpha=1}^{\bar{n}}-\left.\frac{1}{\alpha !} \frac{d^{\alpha}}{d t^{\alpha}} \log z_{t}\left(\Lambda_{12}^{(k)}\right)\right|_{t=0},
$$

and a remainder

$$
\mathscr{R}_{k}\left(\Lambda_{12}^{(k)}\right)=\int_{\sigma}^{1} d t-\frac{(1-t)^{\bar{n}}}{(\bar{n}+1) !}\left\langle\frac{d}{d t} ; \ldots ; \frac{d}{d t}\right\rangle_{t} .
$$

Here $\langle\cdot\rangle_{t}$ is the interacting expectation

$$
\langle\cdot\rangle_{t}=\frac{1}{z_{t}\left(\Lambda_{12}^{(k)}\right)}\left\langle\cdot \chi_{\Lambda_{12}^{\prime(k)}, t}^{\prime} e^{-t \tilde{V}_{12}^{(k)}\left(\Lambda^{(k)}\right)}\right\rangle_{1, \Lambda_{12}^{(k)}}
$$

with $\chi_{\Lambda_{12}^{(k)}, t}^{\prime}$ defined as above replacing $p\left(e_{k}\right)$ with $p\left(t e_{k}\right)$.

We express each $d / d t$ as a sum $\sum_{\gamma}(d / d t)_{\gamma}$, where $(d / d t)_{\gamma}$ acts only on the $t$ before a particular term $V^{(k)}(Y)$ in $\tilde{V}^{(k)}$ or in a particular $\chi$-factor. We cluster expand as before each integral making up the truncated expectation values $\left\langle(d / d t)_{\gamma_{1}}\right.$; $\left.\ldots ;(d / d t)_{\gamma_{\bar{n}+1}}\right\rangle_{t}$. Let $H \subset\{1, \ldots, \bar{n}+1\}$ specify which observables are included in one of the integrals. If $J \in H$ then we have a factor $(d / d t)_{\gamma_{j}}$ in the integral. The partition 
$\left\{\square_{i}\right\}$ of $\Lambda_{12}^{(k)}$ is determined by the sets $Y$ from $e^{-t V^{(k)}(Y)}-1$ factors, and by sets $Y$ from $V^{(k)}(Y)$ factors differentiated down. The expansion takes the form

$$
\left\langle\prod_{j \in H}\left(\frac{d}{d t}\right)_{\gamma_{J}} \chi_{\Lambda_{12}^{\prime}(k), t}^{\prime} e^{-t \tilde{V}^{(k)}\left(A_{12}^{(k)}\right)}\right\rangle_{1 . A_{12}^{(k)}}=\sum_{\left\{X_{\alpha}\right\} \text { filling } A_{12}^{(k)}} \prod_{\beta} g_{3}\left(H_{\beta}, X_{\beta}\right) .
$$

Here $H_{\beta} C H$ specifies which $(d / d t)_{\gamma_{J}}$ have supports intersecting $X_{\beta}$.

The polymer activity $g_{3}$ is essentially the same as $g_{2}$, but with additional observables, namely the $(d / d t)_{\gamma}$-factors determined by $H_{\beta}$. Also, the interaction and characteristic functions have been partially interpolated away, and there are not $W_{5}^{(k)}$-terms.

If $\left|X_{\beta}\right|=1, H_{\beta}=\emptyset$, we write $g_{3}\left(\emptyset, X_{\beta}\right)=1+g_{3}^{\prime}\left(\emptyset, X_{\beta}\right)$ and the above expansion holds again, but without the condition that $\left\{X_{\beta}\right\}$ fill $\Lambda_{12}^{(k)}$. The $\left\{X_{\beta}\right\}$ must cover all cubes connected with the $(d / d t)_{\gamma}, j \in H$. Let us drop the prime, and prove that

$$
\left|g_{3}\left(H_{\beta}, X_{\beta}\right)\right| \leqq\left(e^{\beta}\left(L^{k} \varepsilon / \varepsilon_{0}\right)^{1 / 4-\alpha}\right)^{\left[\left|H_{\beta}\right|+\beta^{\prime}\left|X_{\beta} \backslash H_{\beta}\right|\right]} .
$$

We use $X_{\beta} \backslash H_{\beta}$ to denote the set of cubes with no $(d / d t)_{\gamma_{j}}$ factors, $j \in H_{\beta}$.

The proof of this estimate is similar to the one for $g_{2}$. We mention only the new features. Each factor $V^{(k)}(Y)$ in $\prod_{j \in H_{\beta}}(d / d t)_{\gamma_{j}}$ produces a factor $e^{\beta}\left(L^{k} \varepsilon / \varepsilon_{0}\right)^{1 / 4-\alpha}$ in the final estimate. This is obtained in the Gaussian integration estimate, using the fact that $V^{(k)}(Y)$ is a small polynomial in $A^{(k)}, \phi^{(k)}$. [The restrictions disappear as $t \rightarrow 0$, so $V^{(k)}(Y)$ cannot be replaced by its supremum.] The factors $e^{-t V^{(k)}(Y)}-1$ can be bounded as before, because the coefficient $t$ in front of $V^{(k)}(Y)$ plus a small power of $e_{k}$ easily beat the bounds $A^{(k)}, \phi^{(k)} \leqq c p\left(e_{k}\right)$. Each $t$-derivative of a $\chi$-factor in $\chi_{A_{12}^{(k)}, t}$ gives at least a factor $e^{\beta}\left(L^{k} \varepsilon / \varepsilon_{0}\right)^{1 / 4-\alpha}$. This follows because with $\chi^{\prime}(1, x) \equiv d / d x \gamma(1, x)$, we have

$$
\begin{aligned}
\left|\frac{d}{d t} \chi\left(c p\left(t e_{k}\right), A^{(k)}\right)\right| & =\left|\frac{A^{(k)}}{c p\left(t e_{k}\right)^{2}}\left(\frac{d}{d t} p\left(t e_{k}\right)\right) \chi^{\prime}\left(1, A^{(k)} / c p\left(t e_{k}\right)\right)\right| \\
& \leqq c t^{-1}\left|\chi^{\prime}\left(1, A^{(k)} / c p\left(t e_{k}\right)\right)\right|,
\end{aligned}
$$

and similarly the $n$-th derivative in $t$ of $\chi\left(c p\left(e_{k}\right), A^{(k)}\right)$ is bounded by $t^{-n}$ times a function bounded by a constant and supported in $c_{1} p\left(t e_{k}\right) \leqq\left|A^{(k)}\right| \leqq c_{2} p\left(t e_{k}\right)$. After integration over $A^{(k)}$, we obtain factors $c t^{-n} e^{-c p\left(t_{k}\right)^{2}} \leqq\left(e^{\beta}\left(L^{k} \varepsilon / \varepsilon_{0}\right)^{1 / 4-\alpha}\right)^{n}$. Similar bounds hold for $\phi^{(k)}$. The bound for $H_{\beta}=\emptyset,\left|X_{\beta}\right|=1$ was obtained for $g_{2}$, and the same proof applies here.

Returning to our expansion, let us sum first over $\left\{H_{\gamma}\right\}$, the partition of $H$ determined by the $\left\{X_{\beta}\right\}$. Denote the $X_{\beta}$ 's with $H_{\beta} \neq \emptyset$ by $X_{\gamma}$; the $X_{\beta}$ with $H_{\beta}=\emptyset$ by $Y_{\delta}$. The expansion (5.14.2) becomes

$$
\sum_{\left\{H_{\gamma}\right\} \in \mathscr{P}(H)} \sum_{\left\{X_{\gamma}\right\},\left\{Y_{\delta}\right\} \text { nonoverlapping }} \prod_{\gamma} g_{3}\left(H_{\gamma}, X_{\gamma}\right) \prod_{\delta} g_{3}\left(\emptyset, Y_{\delta}\right) .
$$

Each $X_{\gamma}$ must cover and connect all the $t$-derivatives specified by $H_{\gamma}$. Next we reorganize this expansion in order to extract the truncated expectation values (5.14.2). This involves adding and subtracting terms in a scheme familiar to one in [10]. We insert factors

$$
u(X, Y)=\left\{\begin{array}{llll}
0 & \text { if } & X, Y & \text { overlap } \\
1 & \text { if } & X, Y & \text { do not overlap }
\end{array}\right.
$$


and similarly factors $u\left(X_{1}, X_{2}\right), u\left(Y_{1}, Y_{2}\right)$. We extend the sums over $\left\{X_{\gamma}\right\},\left\{Y_{\delta}\right\}$ to nonoverlapping sets; however the corresponding subsets of $H$ remain the same no duplication of $t$-derivatives. We put $u=1+a$ and expand in the usual manner. This enables us to factor out the normalization $z_{t}\left(\Lambda_{12}^{(k)}\right)$ to obtain

$$
\begin{aligned}
\left\langle\prod_{j \in H}\left(\frac{d}{d t}\right)_{\gamma}\right\rangle_{t}= & \sum_{\left\{H_{\gamma} \in \mathscr{P}(H)\right.} \sum_{\left\{X_{\gamma}\right\}} \sum_{\left(Y_{1}, \ldots, Y_{B}\right)} \\
& \times \frac{1}{B !} \sum_{G} \prod_{\mathscr{L} \in G} a(\mathscr{L}) \prod_{\gamma} g_{3}\left(H_{\gamma}, X_{\gamma}\right) \prod_{\delta=1}^{B} g_{3}\left(\phi, Y_{\delta}\right) .
\end{aligned}
$$

Here $\mathscr{L}$ denotes pairs of clusters (lines) and $G$ runs over graphs of such lines in which each $Y_{\delta}$ is connected directly or indirectly to some $X_{\gamma}$. The connected components of $G$ define a partition of $H$ which corresponds to the partition in the formula

$$
\left\langle\prod_{j \in H}\left(\frac{d}{d t}\right)_{\gamma_{j}}\right\rangle_{t}=\sum_{\left\{H_{\tau}^{\prime}\right\} \in \mathscr{P}(H)} \prod_{\tau}\left\langle\prod_{j \in H_{\tau}^{\prime}}\left[;\left(\frac{d}{d t}\right)_{\gamma_{j}}\right]\right\rangle_{t} .
$$

Thus we have a formula

$$
\begin{aligned}
& \left\langle\prod_{j \in H}\left[;\left(\frac{d}{d t}\right)_{\gamma_{J}}\right]\right\rangle_{t}=\sum_{\left\{H_{\gamma}\right\} \in \mathscr{P}(H)} \sum_{\left\{X_{, \gamma}\right\}} \sum_{\left(Y_{1}, \ldots . Y_{B}\right)} \\
& \times \frac{1}{B !} \sum_{G_{c}} \prod_{\mathscr{L} \in G_{c}} a(\mathscr{L}) \prod_{\gamma} g_{3}\left(H_{\gamma}, X_{\gamma}\right) \prod_{\delta=1}^{B} g_{3}\left(\phi, Y_{\delta}\right),
\end{aligned}
$$

where $G_{c}$ runs over connected graphs involving all clusters $X_{\gamma}, Y_{\delta}$, and hence all of $H$.

We use this to give an expansion for the remainder from the perturbation expansion of the interaction:

$$
\mathscr{R}_{k}\left(\Lambda_{12}^{(k)}\right)=\sum_{X \subset \Lambda_{12}^{(k)}} W_{6}^{(k) \prime}(X) .
$$

Here $W_{6}^{(k) \prime}(X)$ is obtained by summing only over $\left\{X_{\gamma}\right\},\left(Y_{1}, \ldots, Y_{B}\right)$ which fill $X$, summing over $\left\{\gamma_{j}\right\}$ with suppt $(d / d t)_{\gamma_{j}} \subset X$, and integrating over $t$ as in (5.14.2). It is now a standard exercise to estimate the expansion, using (5.14.4). The result is

$$
\left|W_{6}^{(k) \prime}(X)\right| \leqq\left(e^{\beta}\left(L^{k} \varepsilon / \varepsilon_{0}\right)^{1 / 4-\alpha}\right)^{\bar{n}+1+\beta^{\prime}|X|}
$$

(We allow adjustments in $\beta, \alpha, \beta^{\prime}$, keeping them small.)

We make some modifications in the perturbative terms to achieve the standard form of the interaction, $\mathscr{P}_{k+1,10 c}^{L}$. We give random walk expansions for the

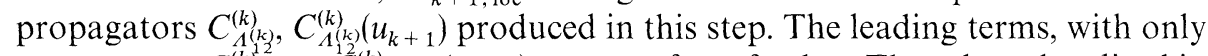
propagators $C_{\Lambda_{12}^{(k)} \text {, loc }}^{(k)^{2}}, C_{\Lambda_{12}^{(k)} \text {, loc }}^{(k)}\left(u_{k+1}\right)$, we transform further. The others, localized in region $X$, have a factor of $e^{-c r\left(e_{k}\right)|X|}$. We also consider as remainders any terms whose order in $\lambda$ and $e$ is greater than $\bar{n}$.

We wish to replace $C_{\Lambda,(k)}^{(k)}$, loc with $C_{\text {loc }}^{(k)}$. Recall that $C_{\Lambda}^{(k)}$ is the Dirichlet inverse to (5.13.2), and we define $C_{\Lambda, \text { loc }}^{(k)}$ by cutting off the kernel when the arguments are separated by $O\left(r\left(e_{k}\right)\right)$. $C_{\text {loc }}^{(k)}$ was defined in (2.9), starting from the inverse to $\partial^{*} \sigma_{k, \text { loc }} \partial$ on the appropriate subspace. The replacement of $C_{\Lambda_{12}^{(k)} \text {, loc }}^{(k)}$ with $C_{\mathbb{Z}^{d}, \text { loc }}^{(k)}$ produces 
terms localized near $\Lambda_{12}^{(k) c}$. These terms are bounded by a small power of coupling constants, $e^{\beta}\left(L^{k} \varepsilon / \varepsilon_{0}\right)^{1 / 4-\alpha}$. To make the replacement of $C_{\mathbb{Z}^{a}, \text { loc }}^{(k)}$ with $C_{\text {loc }}^{(k)}$, note that the former always appears between two operators as $\left(I-Q^{s *} Q\right) C_{\mathbb{Z}^{d}, \text { loc }}^{(h)}\left(I-Q^{*} Q^{s}\right)$. This differs from $C_{\text {loc }}^{(k)}$ by a small, local operator, since after replacing $C_{\mathbb{Z}^{d}, \text { loc }}^{(k)}$ with $C_{\mathbb{Z}^{d}}^{(k)}$, we have an identity

$$
\left(I-Q^{s *} Q\right) C_{\mathbb{Z}^{d}}^{(k)}\left(I-Q^{*} Q^{s}\right)=C^{(k)}=C_{\mathrm{loc}}^{(k)}+O\left(e^{-c r\left(e_{k}\right)}\right) .
$$

Thus after removing some $O\left(e^{-c r\left(e_{k}\right)}\right)$ remainders, we have our standard covariance $C_{\text {loc }}^{(k)}$.

We compose propagators, using also terms from $V_{\text {const }}^{(k)}\left(\Lambda_{8}^{(k)}\right)$. For gauge field propagators, we apply (5.5.11). For scalar field propagators, we use the identity 2.42 from [7]:

$$
\begin{aligned}
G_{k}\left(\square, u_{k+1}\right) & +a_{k}^{2} L^{-2} G_{k}\left(\square, u_{k+1}\right) Q_{k}^{*}\left(u_{k+1}\right) C^{(k)}\left(\square, u_{k+1}\right) \\
& \times Q_{k}\left(u_{k+1}\right) G_{k}\left(\square, u_{k+1}\right)=G_{k+1}^{\eta}\left(\square, u_{k+1}\right) .
\end{aligned}
$$

There are also small $\left(O\left(e^{-c r\left(e_{k}\right)}\right)\right)$ terms involving $G_{k, \text { loc }}\left(u_{k+1}\right)-G_{k}\left(\square, u_{k+1}\right)$, $C_{\text {loc }}^{(k)}\left(u_{k+1}\right)-C^{(k)}\left(\square, u_{k+1}\right)$, and $G_{k+1 . \operatorname{loc}}\left(u_{k+1}\right)-G_{k+1}\left(\square, u_{k+1}\right)$, and boundary terms as above involving $C_{\Lambda_{12}^{(k)}(k) \text { loc }}^{(k)}\left(u_{k+1}\right)-C_{\text {loc }}^{(k)}\left(u_{k+1}\right)$. We end up with scalar field propagators $G_{k+1, \text { loc }}^{\eta}\left(u_{k+1}\right)$. For simplicity we extend the localizations of vertices in all diagrams back to $\bar{\Lambda}_{8}^{(k)}$ (for gauge fields we use a smooth localization function). This produces more boundary terms. Then the terms produced in this step combine with the old terms $V_{\text {const }}^{(k)}\left(\Lambda_{8}^{(k)}\right)$ to produce the full interaction $\mathscr{P}_{k+1 . \operatorname{loc}}^{L}\left(\Lambda_{8}^{(k)}\right)$. Altogether we have written

$$
V_{\text {const }}^{(k)}\left(\Lambda_{8}^{(k)}\right)+\tilde{P}_{k+1}\left(\Lambda_{12}^{(k)}\right)=\mathscr{P}_{k+1.10 c}^{L}\left(\Lambda_{8}^{(k)}\right)+\sum_{X} W_{6}^{(k) \prime \prime}(X) .
$$

If we put $W_{6}^{(k)}(X)=W_{6}^{(k) \prime}(X)+W_{6}^{(k) \prime \prime}(X)$, then $W_{6}^{(k)}(X)$ obeys

$$
\left|W_{6}^{(k)}(X)\right| \leqq \begin{cases}\left(e^{\beta}\left(L^{k} \varepsilon / \varepsilon_{0}\right)^{1 / 4-\alpha}\right)^{\bar{n}+1+\beta^{\prime}|X|}, & \operatorname{dist}\left(X, A_{12}^{(k), c}\right) \geqq r\left(e_{k}\right) \\ \left(e^{\beta}\left(L^{k} \varepsilon / \varepsilon_{0}\right)^{1 / 4-\alpha}\right)^{\beta^{\prime}|X|}, & \text { otherwise. }\end{cases}
$$

We apply a somewhat different procedure to extract the proper perturbative terms from the observable. We integrate by parts in the Gaussian expectation (5.14.1). Each $F_{k, \text { loc }}^{\bar{m}}\left(X_{\sigma_{1}}\right)$ is a polynomial in $A^{(h)}, \phi^{(k)}$; those fields can be contracted via covariances $C_{\Lambda_{12}^{(k)}}^{(k)}$ or $C_{\Lambda_{12}^{(k)}(k)}^{(k)}\left(u_{k+1}\right)$ to other observables, to $\chi_{A_{12}^{(k)}}^{\prime}$, or to the interaction. After each integration by parts, we replace the covariance by $C_{\text {loc }}^{(k)}$ or $C_{\text {loc }}^{(h)}\left(u_{k+1}\right)$ and give a random walk expansion for the difference. For each term, let $\bar{X}$ be the union of the cubes covering the $X_{\sigma_{1}}$ and the regions from the random walk expansion. A connected component of $\bar{X}$ is called complete if a contraction to $\chi_{\left.A_{12}^{\prime}\right)}^{\prime}$ occurs, if a term from the random walk expansion occurs, if at least $\bar{m}+1$ interactions have been differentiated down, or if the term is constant (all legs contracted). We stop integrating by parts fields in complete components of $\bar{X}$. After sufficiently many integrations by parts, all components of $\bar{X}$ will be complete.

We break up the observable according to the connected components of $\bar{X}$. The components containing contractions to $\chi_{\Lambda_{12}^{(k)}}^{\prime}$, terms from the random walk expansions, or at least $\bar{m}+1$ interactions are called remainder components $\left\{X_{r}\right\}$. The other components are called constant components $\left\{X_{c}\right\}$, since the observable 
there is independent of $A^{(k)}, \phi^{(k)}$. We can arrange the construction so that the $\left\{X_{c}\right\}$ are determined once the remainder components are specified. Summing all possible diagrams in $X_{c}$ gives the observable for the next step there, $F_{k+1, \text { loc }}^{L}\left(X_{c}\right)$. Summing all terms in $X_{r}$ gives an observable $F_{k, \text { rem }}\left(X_{r}\right)$. Then the result of the integration by parts is

$$
\frac{z_{F}}{z}=\left\langle\prod_{\sigma_{1}} F_{k, \text { loc }}^{\bar{m}}\left(X_{\sigma_{1}}\right)\right\rangle_{1}=\sum_{\left\{X_{r}\right\}} \prod_{c} F_{k+1, \operatorname{loc}}^{L}\left(X_{c}\right)\left\langle\prod_{r} F_{k \cdot \mathrm{rem}}\left(X_{r}\right)\right\rangle_{1},
$$

where $\langle\cdot\rangle_{1}$ is the interacting expectation at $t=1$.

Having extracted the desired perturbative terms $F_{k+1,10 c}^{L}\left(X_{c}\right)$, we need to finish the calculation of the remainders by giving a cluster expansion for $\left\langle\prod_{r} F_{k \cdot \mathrm{rem}}\left(X_{r}\right)\right\rangle_{1}$, with appropriate bounds. We use essentially the same expansion as before, Mayerexpanding $V^{(k)}(Y)$ 's and interpolating the Gaussian measure. Finally the polymer expansion $u=1+a$ permits us to factor out the normalization. Without going into details, it is clear that the result can be written in the following form:

$$
\left\langle\prod_{\sigma_{1}} F_{k, \operatorname{loc}}^{\bar{m}}\left(X_{\sigma_{1}}\right)\right\rangle_{1}=\sum_{\left\{X_{r^{\prime}}\right\}} \prod_{r^{\prime}} G_{k}\left(X_{r^{\prime}}\right) \prod_{c: X_{c} \Phi_{,}, X_{r^{\prime}}} F_{k+1,10 \mathrm{loc}}^{L}\left(X_{c}\right) .
$$

The $X_{r^{\prime}}$ are disjoint, and each one covers at least one $X_{\sigma_{1}}$, the support of one of the observables $F_{k, \text { loc }}^{\bar{m}}$.

The main source of concern in estimating $G_{k}\left(X_{r^{\prime}}\right)$ is that we only have bounds $\left|F_{h .10 c}\left(X_{\sigma_{1}}\right)\right| \leqq c\left(L^{k} \varepsilon\right)^{-m(c)} e^{-m^{\prime}(c)}$, coming from our estimates on perturbation expansions of observables; similarly for $F_{k+1, \operatorname{loc}}^{L}\left(X_{c}\right)$. Here $m(c), m^{\prime}(c)$ depend on the terms in $F$ in $X_{\sigma_{1}}$ or $X_{c}$. By performing sufficiently many integrations by parts, we have arranged for enough small factors to beat these large factors in the remainder terms (at least if $X_{r^{\prime}}$ is not at the boundary of $\Lambda_{12}^{(k)}$ ). Near the boundary we have potentially large covariances $C_{\Lambda_{12}^{(k)}(\text { loc }}^{(k)}-C_{\text {loc }}^{(k)}$ or $C_{\Lambda^{(k)}, \text { loc }}^{\left(u_{k+1}\right)}\left(u_{k+1}-C_{\text {loc }}^{(k)}\left(u_{k+1}\right)\right.$, so we make use of the proximity to $\Lambda_{12}^{(k) c}$ to provide the necessary convergence. These considerations lead to the following estimate:

$$
\begin{aligned}
\left|G_{k}(X)\right| \leqq & c(F(X))\left(e^{\beta}\left(L^{k} \varepsilon / \varepsilon_{0}\right)^{1 / 4-\alpha}\right)^{\beta^{\prime}\left|X \backslash \cup X X_{c}\right|} \\
& \times \prod_{X_{\sigma_{1}} c X: \operatorname{dist}\left(X_{\sigma_{1}} \cdot A_{12}^{(h) c}\right)<r\left(e_{k}\right)}\left[c\left(L^{k} \varepsilon\right)^{-m(c)} e^{-m^{\prime}(c)}\right] .
\end{aligned}
$$

To summarize the results of this section, we have

$$
\begin{aligned}
e^{-V_{\text {const }}^{(k)}\left(\Lambda_{8}^{(k)}\right)} & \sum_{\left\{X_{\alpha}\right\}} \prod_{\alpha} g_{2}\left(X_{\alpha}\right) \\
= & \sum_{\left\{X_{\alpha}\right\} \text { overlapping } A_{11}^{(k) c}} \prod_{\alpha} g_{2}\left(X_{\alpha}\right) \sum_{\left\{X_{r^{\prime}}\right\}} \prod_{r^{\prime}} G_{k}\left(X_{r^{\prime}}\right) \\
& \times \prod_{c: X_{c} \Psi_{r^{\prime}} X_{X^{\prime}}} F_{k+1,10 c}^{L}\left(X_{c}\right) \exp \left(-\mathscr{P}_{k+1.10 c}^{L}\left(\Lambda_{8}^{(k)}\right)-\sum_{X} W_{6}^{(k)}(X)\right) .
\end{aligned}
$$

\subsection{Second Mayer Expansion and Scaling}

In this section we recover the induction hypothesis for $k+1$ instead of $k$, and write a formula for the hole functional $g_{k+1}\left(X_{\omega}\right)$. First we Mayer-expand the irrelevant $W_{6}^{(k)}$ terms:

$$
\exp \left(-\sum_{X} W_{6}^{(k)}(X)\right)=\sum_{S_{6}} \prod_{X \in S_{6}}\left(e^{-W_{6}^{(k)}(X)}-1\right)
$$


We define

$$
\Lambda_{13}^{(k)}=\Lambda_{12}^{(k)} \bigcup_{X \in S_{6}} X \backslash \bigcup_{r^{\prime}} X_{r^{\prime}} \bigcup_{c: X_{c} \cap X \neq \emptyset, X \in S_{6}} X_{c}
$$

it is a region now completely free of irrelevant terms. We write

$$
\prod_{c: X_{c} \notin \cup Y^{\prime} X_{r^{\prime}}} F_{h+1.1 \mathrm{loc}}^{L}\left(X_{c}\right)=\prod_{c^{\prime}} F_{k+1, \operatorname{loc}}^{L}\left(X_{c^{\prime}}\right) \prod_{\sigma^{\prime}} F_{k+1, \operatorname{loc}}^{L}\left(X_{\sigma^{\prime}}\right),
$$

where $\left\{\sigma^{\prime}\right\}=\left\{c: X_{c} \subset \Lambda_{13}^{(k)}\right\}$, and $\left\{c^{\prime}\right\}$ are the rest.

By inserting (5.14.5), (5.15.1), (5.15.2) in (5.12.8), we obtain the final form of the density $\varrho_{k+1}^{L}(v, \psi)$.

We now scale this density from $T_{L}^{(k+1)}$ to $T_{1}^{(k+1)}$, putting $\psi^{L}(y)=L^{-(d-2) / 2}$ $\psi^{1}\left(L^{-1} y\right)$. If we define

$$
\varrho\left(v, \psi^{1}\right)=\exp \left[-\frac{d-2}{2}(\log L)\left|T_{1}^{(k+1)}\right|\right] \varrho^{L}\left(v, L^{-(d-2) / 2} \psi^{1}\right),
$$

then the integral of $\varrho\left(v, \psi^{1}\right)$ is equal to the integral of $Q^{L}\left(v, \psi^{L}\right)$. Thus we define the $(k+1)$ th normalizing energy to be

$$
\mathscr{E}_{k+1}=\mathscr{E}_{k}+E^{(k)}+\frac{d-2}{2}(\log L)\left|T_{1}^{(k+1)}\right|
$$

Let us describe how the scaling affects a few of the objects that will be needed in the next step. Defining $f^{(k+1)}(p)=\left(i e_{k+1}\right)^{-1} \log v(p)$, we have that

$$
e_{k} \eta L^{-2} \mathscr{D}_{k+1.10 \mathrm{c}}^{\eta} \partial^{\eta *} Q_{k+1}^{e *} f=e_{k+1} L^{-1} \eta \mathscr{D}_{k+1.10 \mathrm{c}} \partial^{L^{-1} \eta *} Q_{k+1}^{e *} f^{(k+1)},
$$

and thus in $\Lambda_{6}^{(k) *}$ we have

$$
u_{k+1, b}=\left(Q^{s *} v\right) \exp \left[-i e_{k} L^{-1} \eta \mathscr{D}_{k+1, \operatorname{loc}} \partial^{L^{-1} \eta *} Q_{k+1}^{e *} f^{(k+1)}\right]
$$

as in the induction hypothesis (4.2). The quadratic forms become

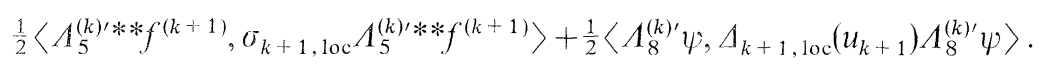

The interaction and observables are scaled and written as $\mathscr{P}_{k+1,10 c}\left(\Lambda_{8}^{(k)}\right)$ and $F_{k+1, \text { loc }}\left(X_{\sigma^{\prime}}\right)$, respectively. Propagators and vertices appear scaled to the $L^{-1} \eta$ lattice. The scaled form of the normalization factors is given in (4.6), (4.9).

Let $\left\{X_{\omega^{\prime}}\right\}$ be the components of $\Lambda_{13}^{(k) c}$, and let $X_{\omega^{\prime}}$ also specify $\Lambda_{\alpha}^{(k) c} \cap X_{\omega^{\prime}}$ and a collection $\left\{X_{\omega}\right\}$ of sets from the previous step. We exhibit the factorization of most of the terms in $\varrho_{k+1}(v, \psi)$ by writing

$$
\begin{aligned}
& \varrho_{k+1}(v, \psi)=\left.\sum_{\left\{X_{\left.()^{\prime}\right\}}\right.} \int \prod_{j=0}^{k} d u^{(j)}\right|_{\left.A_{1 \%}^{(j)}\right)} Q_{k+1}^{\prime}\left(v, \psi,\left\{X_{\omega^{\prime}}\right\},\left\{u^{(j)}\right\}\right) \\
& \varrho_{k+1}^{\prime}\left(v, \psi,\left\{X_{\omega^{\prime}}\right\},\left\{u^{(j)}\right\}\right) \\
& =\chi_{k+1, A_{0}^{(k)}, \prod_{()^{\prime}}} g_{k+1}\left(X_{\left(0^{\prime}\right.}\right) \prod_{\sigma^{\prime}} F_{k+1.10 c}\left(X_{\sigma^{\prime}}\right) \prod_{j=0}^{k}\left[Z_{\Lambda_{10}^{(j)) \times c}}^{(j)} Z_{\Lambda_{10}^{(j)}}^{(j)}\left(u_{k+1}\right)\right]
\end{aligned}
$$

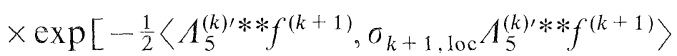

$$
\begin{aligned}
& \left.-\frac{1}{2}\left\langle\Lambda_{8}^{(k) \prime} \psi, \Lambda_{k+1, \operatorname{loc}}\left(u_{k+1}\right) \Lambda_{8}^{(k) \prime} \psi\right\rangle-\mathscr{P}_{k+1.1 \mathrm{loc}}\left(\Lambda_{8}^{(k)}\right)-\mathscr{E}_{k+1}\right],
\end{aligned}
$$


which is in the form of our original induction hypothesis, (4.1). The hole functional has the expression

$$
\begin{aligned}
& g_{k+1}\left(X_{\omega^{\prime}}\right)=\sum_{S_{4}, \check{\sigma}_{1}, \check{A}_{9}^{(k)} \cap X_{\omega^{\prime}},\left\{X_{x}\right\},\left\{X_{\left.r^{\prime}\right\}}, S_{6} \text { compatible with } X_{\omega} .\right.} \\
& \times\left.\int d \phi^{(k)}\right|_{A_{10}^{(k) c} \cap X_{\left(0^{\prime}\right.}} \delta_{\mathrm{Ax}, A_{10}^{(k), c} \cap X_{()^{\prime}}}\left(u^{(k)}\right) \delta_{A_{1}^{(h) * * c} \cap X_{\omega}}\left(v / Q u^{(k)}\right)
\end{aligned}
$$

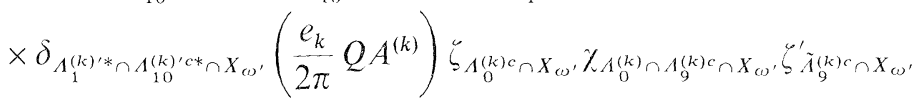

$$
\begin{aligned}
& \times \prod_{\omega} g_{k}\left(X_{\omega}\right) \prod_{\sigma \notin \delta, X_{\sigma} c \cdot X_{(\omega,}} F_{k \cdot \operatorname{loc}}^{\prime}\left(X_{\sigma}\right) \prod_{\left(j, x_{j}, X\right) \in S_{4}}\left(e^{-W_{4, j}^{(k)}(x, 1, X)}-1\right) \\
& \times \prod_{\alpha} g_{2}\left(X_{\alpha}\right) \prod_{r^{\prime}} G_{k}\left(X_{r^{\prime}}\right) \prod_{X \in S_{6}}\left(e^{-W_{6}^{(k)}(X)}-1\right) \prod_{c^{\prime}: X_{c^{\prime}} \subset X_{\left.\omega^{\prime}\right)}} F_{k+1, \operatorname{loc}}\left(X_{c^{\prime}}\right) \\
& \times \exp \left[-\sum_{i=1}^{8} \mathscr{Q}_{i}\left(X_{\omega^{\prime}}\right)-\mathscr{P}_{k \cdot 1 \mathrm{oc}}\left(\Lambda_{8}^{(k-1)}, \Lambda_{8}^{(k) c} \cap X_{\omega^{\prime},} \tilde{u}_{k+1}\right)\right. \\
& -R^{(k)}\left(\Lambda_{8}^{(k) c} \cap X_{\omega 0^{\prime}}, u_{k+1}, \theta_{k} H_{h \cdot \operatorname{loc}} A^{(k)}\right) \\
& \left.-Q^{(k)}\left(\Lambda_{8}^{(k) c} \cap X_{()^{\prime}}, u_{k+1}, \theta_{k} H_{k \cdot 1 \mathrm{loc}} A^{(k)}\right)\right] \text {. }
\end{aligned}
$$

Compatibility means that the summations run over sets associated only with $X_{\omega^{\prime}}$ and that the sets would have given us $X_{\omega^{\prime}}$ in the course of our constructions. Specifically, this implies a certain "density" of terms leading to convergence factors, and compatibility of the sets with the layered structure imposed by the $\Lambda_{\alpha}^{(k)}$.

We have discussed the estimates on many of the elements of the expansion in $g_{k+1}$. However, we cannot complete the estimates until after extracting convergence from the large field conditions. This is accomplished only after integrating over the final $v, \psi$ in the last step. These problems, and the problem of decoupling of the final fields, will be considered in a subsequent paper.

\section{References}

1. Bałaban, T., Imbrie, J., Jaffe, A.: Exact renormalization group for gauge theories. In: Progress in gauge field theory. Cargese 1983. Lehmann, G., 't Hooft, G., Jaffe, A., Mitter, P., Singer, I., Stora, R. (eds.). New York: Plenum 1984

2. Bałaban, T., Imbrie, J., Jaffe, A.: Renormalization of the Higgs model: Minimizers, propagators, and the stability of mean field theory. Commun. Math. Phys. 97, 299-329 (1985)

3. Bałaban, T., Brydges, D., Imbrie, J., Jaffe, A.: The mass gap for Higgs models on a unit lattice. Ann. Phys. 158, 281-319 (1984)

4. Imbrie, J.: Renormalization group methods in gauge field theories. In: Critical phenomena, random systems, gauge theories. Les Houches 1984. Osterwalder, K., Stora, R. (eds.). Amsterdam: North-Holland 1986

5. Bałaban, T.: (Higgs) ${ }_{2,3}$ quantum fields in a finite volume. III. Renormalization. Commun. Math. Phys. 88, 411-445 (1983)

6. Bałaban, T.: Regularity and decay of lattice Green's functions. Commun. Math. Phys. 89, $571-597(1983)$

7. Bałaban, T.: (Higgs) $)_{2,3}$ quantum fields in a finite volume. I. A lower bound. Commun. Math. Phys. 85, 603-636 (1983)

8. Bałaban, T.: (Higgs) 2,3 quantum fields in a finite volume. II. An upper bound. Commun. Math. Phys. 86, 555-594 (1983) 
9. Glimm, J., Jaffe, A., Spencer, T.: The particle structure of the weakly coupled $\mathscr{P}(\phi)_{2}$ model and other applications of high temperature expansions. In: Constructive quantum field theory. Lecture Notes in Physics, Vol. 25. Velo, G., Wightman, A. (eds.). Berlin, Heidelberg, New York: Springer 1973

10. Gawedzki, K., Kupiainen, A.: Renormalization group for a critical lattice model. Effective interactions beyond the perturbation expansion in bounded spins approximation. Commun. Math. Phys. 88, 77-94 (1983)

Communicated by A. Jaffe

Received March 24, 1987; in revised form May 22, 1987 
